\title{
Some recent results on representations of $p$-adic special orthogonal groups
}

\author{
Jean-Loup Waldspurger
}

\section{Motivation and results}

1.1. Global motivation. Let $V$ be a vector space over $\mathbb{Q}$, of finite dimension $d$, and let $q$ be a non-degenerate quadratic form on $V$. We suppose given an orthogonal decomposition $V=D \oplus W$, where $D$ is a line. Denote by $G$, resp. $H$, the special orthogonal group of $V$, resp. $W$. The group $H$ is a subgroup of $G$, it is the subgroup of elements which act by identity on $D$. We assume, for instance, $d$ even, and, to simplify, $d \geq 4$.

Denote by $\mathbb{A}$ the ring of adeles of $\mathbb{Q}$. The group $G(\mathbb{A})$ acts by right translations on the space $L^{2}(G(\mathbb{Q}) \backslash G(\mathbb{A}))$ of square-integrable functions on $G(\mathbb{Q}) \backslash G(\mathbb{A})$. Consider a closed subspace $\mathcal{V} \subset L^{2}(G(\mathbb{Q}) \backslash G(\mathbb{A}))$, invariant by $G(\mathbb{A})$, such that the representation $\pi$ of $G(\mathbb{A})$ on $\mathcal{V}$ is irreducible (there is no closed invariant subspace distinct from $\mathcal{V}$ and $\{0\})$. Such representation $\pi$ is called automorphic of the discrete spectrum. The quotient $G(\mathbb{Q}) \backslash G(\mathbb{A})$ is not compact in general, but we can define the notion of "rapid decay" for a function on $G(\mathbb{Q}) \backslash G(\mathbb{A})$. Assume that the subset $\mathcal{V}^{0}$ of elements of rapid decay of $\mathcal{V}$ is dense in $\mathcal{V}$. Then we say that $\pi$ is cuspidal. Let $\pi$ be such automorphic cuspidal irreducible representation of $G(\mathbb{A})$ on a subspace $\mathcal{V} \subset L^{2}(G(\mathbb{Q}) \backslash G(\mathbb{A}))$. Similarly, let $\sigma$ be an automorphic cuspidal irreducible representation of $H(\mathbb{A})$ on a subspace $\mathcal{W} \subset L^{2}(H(\mathbb{Q}) \backslash H(\mathbb{A}))$. For $\varphi \in \mathcal{V}^{0}$ and $\psi \in \mathcal{W}^{0}$, we define the integral

$$
J(\psi, \varphi)=\int_{H(\mathbb{Q}) \backslash H(\mathbb{A})} \overline{\psi(h)} \varphi(h) d h .
$$

It is absolutely convergent and define a sesquilinear form $J$ on $\mathcal{W}^{0} \times \mathcal{V}^{0}$. A conjecture of Gross and Prasad ([GP], conjecture 14.8) says (in particular) that, if this sesquilinear form is non-zero, then a special value of some $L$-function is non-zero. What is this $L$-function? Of course, the notion of automorphic representation of the discrete spectrum is defined for all reductive groups over $\mathbb{Q}$ (some minor modifications are needed when the

(C) 2011 International Press 
group has infinite center). Using a conjecture of Langlands, we can associate to $\pi$ a representation $\boldsymbol{\pi}$ of the group $G L(d, \mathbb{A})$ that is not always an automorphic representation of the discrete spectrum, but is induced in a certain sense of such representation of a subgroup of $G L(d, \mathbb{A})$. We can consider $\boldsymbol{\pi}$ as a set $\left\{\boldsymbol{\pi}_{1}, \ldots, \boldsymbol{\pi}_{k}\right\}$, where, for all $i=1, \ldots, k, \boldsymbol{\pi}_{i}$ is an automorphic representation of the discrete spectrum of $G L\left(d_{i}, \mathbb{A}\right)$, with $\boldsymbol{\pi}_{i} \neq \boldsymbol{\pi}_{i^{\prime}}$ if $i \neq i^{\prime}$, and $d=d_{1}+\cdots+d_{k}$. Similarly, we associate to $\sigma$ a representation $\boldsymbol{\sigma}$ of $G L(d-2, \mathbb{A})$, that we can consider as a set $\left\{\boldsymbol{\sigma}_{1}, \ldots, \boldsymbol{\sigma}_{l}\right\}$ (the group $G L(d-2)$ appears here for reasons that we will explain in 1.3). Jacquet, Piatetski-Shapiro and Shalika have defined a function $L(\boldsymbol{\pi} \times \boldsymbol{\sigma}, s)$ depending of a complex parameter $s$. The special value appearing in the Gross-Prasad conjecture is $L(\boldsymbol{\pi} \times \boldsymbol{\sigma}, 1 / 2)$. A more precise conjecture, due to Ichino and Ikeda $([\mathrm{II}])$, gives an exact formula expressing the square $|J(\psi, \varphi)|^{2}$ as the product of $L(\boldsymbol{\pi} \times \boldsymbol{\sigma}, 1 / 2)$ with more technical, but more elementary terms. Cf. 2.10 for a precise statement. When $\pi$ and $\sigma$ have arithmetical properties, such a formula implies rationality properties of the special value of the $L$-function.

A first problem is to understand when $J$ is non-zero. The group $G(\mathbb{A})$ is a subgroup of $\prod_{v} G\left(\mathbb{Q}_{v}\right)$, with a suitable topology, where $v$ describes the places of $\mathbb{Q}$, that is the set of prime numbers plus the infinite place $v=\infty$ for which $\mathbb{Q}_{\infty}=\mathbb{R}$. In a suitable sense, we can decompose $(\pi, \mathcal{V})$ as a tensor product $\otimes_{v}\left(\pi_{v}, \mathcal{V}_{v}\right)$ where, for all $v, \pi_{v}$ is an irreducible unitary representation of $G\left(\mathbb{Q}_{v}\right)$ in an Hilbert space $\mathcal{V}_{v}$. Instead of $\mathcal{V}_{v}$, it is convenient to consider the dense subspace $E_{\pi_{v}}$ of smooth vectors of $\mathcal{V}_{v}$. For $v=p$ a prime, $E_{\pi_{p}}$ is the subspace of the elements $e \in \mathcal{V}_{p}$ such that the stabilizer $\left\{g \in G\left(\mathbb{Q}_{p}\right) ; \pi_{p}(g) e=e\right\}$ is open. This subspace is invariant by $G\left(\mathbb{Q}_{p}\right)$. Of course, the dimension of $E_{\pi_{p}}$ is infinite in general. Similarly, we decompose $(\sigma, \mathcal{W})$ as a tensor product $\otimes_{v}\left(\sigma_{v}, \mathcal{W}_{v}\right)$. We see that, for all $v$, there exists a sesquilinear form $J_{v}$ on $E_{\sigma_{v}} \times E_{\pi_{v}}$ such that $J=\prod_{v} J_{v}$ in a certain sense. Because of the integral definition of $J$, each form $J_{v}$ satisfies the relation

$$
J_{v}\left(\sigma_{v}(h) e^{\prime}, \pi_{v}(h) e\right)=J_{v}\left(e^{\prime}, e\right)
$$

for all $e \in E_{\pi_{v}}, e^{\prime} \in E_{\sigma_{v}}, h \in H\left(\mathbb{Q}_{v}\right)$. Consider the space $\operatorname{Hom}_{H\left(\mathbb{Q}_{v}\right)}\left(E_{\pi_{v}}\right.$, $\left.E_{\sigma_{v}}\right)$. Its elements are the linear applications $l_{v}: E_{\pi_{v}} \rightarrow E_{\sigma_{v}}$ satisfying the relation $l_{v}\left(\pi_{v}(h) e\right)=\sigma_{v}(h)\left(l_{v}(e)\right)$ for all $e \in E_{\pi_{v}}$ and $h \in H\left(\mathbb{Q}_{v}\right)$. To each such $l_{v}$, we can associate the sesquilinear form $J^{l_{v}}$ on $E_{\sigma_{v}} \times E_{\pi_{v}}$ defined by

$$
J^{l_{v}}\left(e^{\prime}, e\right)=\left(e^{\prime}, l_{v}(e)\right)
$$

for all $e^{\prime} \in E_{\sigma_{v}}$ and $e \in E_{\pi_{v}}$, where the product of the right member is the hermitian product on $\mathcal{W}_{v}$. The form $J^{l_{v}}$ satisfies the relation (1). We see easily that the application $l_{v} \mapsto J^{l_{v}}$ is a bijection between $\operatorname{Hom}_{H\left(\mathbb{Q}_{v}\right)}\left(E_{\pi_{v}}, E_{\sigma_{v}}\right)$ and the space of sesquilinear forms on $E_{\sigma_{v}} \times E_{\pi_{v}}$ satisfying (1). In particular, there exists $l_{v} \in \operatorname{Hom}_{H\left(\mathbb{Q}_{v}\right)}\left(E_{\pi_{v}}, E_{\sigma_{v}}\right)$ such that $J_{v}=J^{l_{v}}$. Of course, if $J \neq 0$, then $J_{v} \neq 0$ and $l_{v} \neq 0$. So a necessary condition such that $J \neq 0$ is that, for all $v$, the space $\operatorname{Hom}_{H\left(\mathbb{Q}_{v}\right)}\left(E_{\pi_{v}}, E_{\sigma_{v}}\right)$ is not zero. 
The present formulation of the Ramanujan-Petersson conjecture says that if $\pi \simeq \otimes_{v} \pi_{v}$ is an irreducible automorphic and cuspidal representation of a group $G L(n, \mathbb{A})$, then all the components $\pi_{v}$ are tempered. I will define later this notion. For our group $G$, this property is not true. The Arthur's conjecture says (among other things) what are the cuspidal automorphic representations $\pi$ of $G(\mathbb{A})$ for which we can hope their components are tempered. The condition is that the elements $\boldsymbol{\pi}_{i}$ of $\boldsymbol{\pi}=\left\{\boldsymbol{\pi}_{1}, \ldots, \boldsymbol{\pi}_{k}\right\}$ are cuspidal automorphic representations, and not only automorphic representations of the discrete spectrum. In any case, the automorphic representations whose all components are (conjecturally) tempered are of special interest. So it's reasonable to restrict, at the present time, our local problem to tempered representations.

1.2. The local problem. Now, let $F$ be $\mathbb{Q}_{p}$ for a prime $p$, or more generally, let $F$ be a finite extension of $\mathbb{Q}_{p}$. Let $V=D \oplus W, G$ and $H$ as above except that the base field is now $F$ and not $\mathbb{Q}$. Let $\pi$ be a smooth irreducible representation of $G(F)$ in a complex space $E_{\pi}$ and let $\sigma$ be a smooth irreducible representation of $H(F)$ in a complex space $E_{\sigma}$. We consider the space $\operatorname{Hom}_{H(F)}\left(E_{\pi}, E_{\sigma}\right)$. The first result is the following, due to Aizenbud, Gourevitch, Rallis and Schiffmann ([AGRS]).

TheOREM. $\operatorname{dim}_{\mathbb{C}}\left(\operatorname{Hom}_{H(F)}\left(E_{\pi}, E_{\sigma}\right)\right) \leq 1$.

We denote by $m(\pi, \sigma)$ this dimension. Introduce the contragredient $\check{\pi}$ of $\pi$. That is the transpose representation of $\pi$ in the subspace $E_{\breve{\pi}}$ of smooth vectors of the dual $E_{\pi}^{*}$. For $e \in E_{\pi}$ and $\check{e} \in E_{\check{\pi}}$, we define the coefficient $c_{e, \check{e}}(g)=<\pi(g) e, \check{e}>$ for $g \in G(F)(<., .>$ is the natural pairing between $E_{\pi}$ and its dual). We say that $\pi$ is tempered if, for all $e$ and $\check{e}$, the function $c_{e, \check{e}}$ is of moderate growth, in a suitable sense. The same definition holds for $\sigma$. The central problem of my paper is to compute $m(\pi, \sigma)$ for $\pi$ and $\sigma$ tempered.

In fact, it's more convenient to consider two pairs $\left(V_{i}, W_{i}\right)$ and $\left(V_{a}, W_{a}\right)$ instead of one pair $(V, W)$. We define the discriminant $\delta \in F^{\times} / F^{\times, 2}$ of $q$ (or of $(V, q)$ ) by $\delta=(-1)^{[d / 2]} \operatorname{det}(q)$, where the determinant is computed using any basis of $V$ and $[d / 2]$ is the integral part of $d / 2$ (we have assumed $d$ even in 1.1 , but the same formula holds if $d$ is odd). Recall that, for a given dimension and a given discriminant, there exists exactly two classes of equivalence of spaces provided with quadratic forms, of the given dimension and the given discriminant. We consider two such spaces $V_{i}$ and $V_{a}$, with quadratic forms $q_{i}$ and $q_{a}$, of the same even dimension $d$ and the same discriminant $\delta$, that are not equivalent. We assume given an orthogonal decomposition $V_{i}=D \oplus W_{i}$. Then we can decompose similarly $V_{a}=D \oplus W_{a}$ (with the same quadratic form on $D$ ). It's easy to prove that $W_{i}$ and $W_{a}$, provided with the restrictions of $q_{i}$ and $q_{a}$, have the same dimension and discriminant, but are not equivalent. We denote the four special orthogonal groups by $G_{i}, G_{a}, H_{i}$ and $H_{a}$. 
1.3. Parametrization of the tempered representations. Let $\bar{F}$ be an algebraic closure of $F$. Denote by $W_{F}$ the Weil group of $F$. That is the subgroup of the elements of the Galois group $\operatorname{Gal}(\bar{F} / F)$ that act by an entire power of the Frobenius on the residue field of $\bar{F}$ (the topology of $W_{F}$ is finer that the induced topology). Let $W_{D F}=W_{F} \times S L(2, \mathbb{C})$ be the Weil-Deligne group. Assume again that the dimension $d=\operatorname{dim}(V)$ is even. Then $d^{\prime}=\operatorname{dim}(W)=d-1$ is odd. We introduce the symplectic group $S p\left(d^{\prime}-1, \mathbb{C}\right) \subset G L\left(d^{\prime}-1, \mathbb{C}\right)$. Consider the continuous homomorphisms $\varphi: W_{D F} \rightarrow S p\left(d^{\prime}-1, \mathbb{C}\right)$ such that:

- the composed homomorphism

$$
W_{D F} \stackrel{\varphi}{\rightarrow} S p\left(d^{\prime}-1, \mathbb{C}\right) \rightarrow G L\left(d^{\prime}-1, \mathbb{C}\right)
$$

is a semi-simple representation of $W_{D F}$;

- the restriction of $\varphi$ to $S L(2, \mathbb{C})$ is algebraic.

The group $S p\left(d^{\prime}-1, \mathbb{C}\right)$ acts by conjugacy on the set of such homomorphisms. Denote by $\Phi^{\text {orth }}\left(d^{\prime}\right)$ the set of conjugacy classes. Consider now the subset of $\varphi$ as above, such that $\varphi\left(W_{F}\right)$ is a relatively compact subgroup of $S p\left(d^{\prime}-1, \mathbb{C}\right)$. Denote by $\Phi_{\text {temp }}^{\text {orth }}\left(d^{\prime}\right)$ the set of conjugacy classes in this subset.

Denote by $\sharp$ one of the two indices $i$ or $a$. The first assertion of a local conjecture of Langlands is that, to each $\varphi \in \Phi_{\text {temp }}^{\text {orth }}\left(d^{\prime}\right)$, is associated a finite set $\Pi^{H_{\sharp}}(\varphi)$ of smooth tempered irreducible representations of $H_{\sharp}(F)$, called an $L$-packet, and that the set of smooth tempered irreducible representations of $H_{\sharp}(F)$ is exactly the disjoint union of those $L$-packets $\Pi^{H_{\sharp}}(\varphi)$, when $\varphi$ describes $\Phi_{\text {temp }}^{\text {orth }}\left(d^{\prime}\right)$.

Fix $\varphi$ in $\Phi_{t e m p}^{\text {orth }}\left(d^{\prime}\right)$ (more exactly, $\varphi$ is an homomorphism whose class is in this set). Denote by $S(\varphi)$ the centralizer of the image of $\varphi$ in $S p\left(d^{\prime}-1, \mathbb{C}\right)$, and by $S(\varphi)^{0}$ the identity component of $S(\varphi)$. The group $S(\varphi) / S(\varphi)^{0}$ is abelian, isomorphic to a finite product of copies of $\mathbb{Z} / 2 \mathbb{Z}$. Let $\Pi(\varphi)$ be the disjoint union of $\Pi^{H_{i}}(\varphi)$ and $\Pi^{H_{a}}(\varphi)$ and let $\left(S(\varphi) / S(\varphi)^{0}\right)^{\wedge}$ be the dual group of $S(\varphi) / S(\varphi)^{0}$. Then the conjecture says there is a bijection

$$
\begin{aligned}
\left(S(\varphi) / S(\varphi)^{0}\right)^{\wedge} & \rightarrow \Pi(\varphi) \\
\epsilon & \mapsto \pi(\varphi, \epsilon) .
\end{aligned}
$$

This bijection must satisfy some properties related to the theory of endoscopy and is uniquely determined by these properties.

The conjecture for the groups $G_{i}$ and $G_{a}$ is similar. The dimension $d$ is even. The discriminant $\delta$ is an element of $F^{\times} / F^{\times 2}$, which defines a quadratic character of $W_{F}$, also denoted by $\delta$. We introduce the complex orthogonal group $O(d, \mathbb{C})$ and we consider the homorphisms $\varphi: W_{F} \rightarrow$ $O(d, \mathbb{C})$ satisfying the same conditions as above and the following. The sequence

$$
W_{F} \rightarrow W_{D F} \stackrel{\varphi}{\rightarrow} O(d, \mathbb{C}) \stackrel{\text { det }}{\rightarrow}\{ \pm 1\}
$$

defines a quadratic character $\delta_{\varphi}$. We assume $\delta_{\varphi}=\delta$. We denote $\Phi^{\text {orth }}(d, \delta)$ the set of conjugacy classes for the action of $S O(d, \mathbb{C})$ (and not $O(d, \mathbb{C})$ ) in 
the set of such $\varphi$. We define as above the subset $\Phi_{\text {temp }}^{\text {orth }}(d, \delta)$. As previously, we conjecture that to each $\varphi \in \Phi_{\text {temp }}^{\text {orth }}(d, \delta)$ is associate a finite $L$-packet $\Pi^{G_{\sharp}}(\varphi)$ of smooth tempered irreducible representations of $G_{\sharp}(F)$ (for $\sharp=i$ or $a$ ). The set of smooth tempered irreducible representations of $G_{\sharp}(F)$ must be the disjoint union of the packets $\Pi_{\sharp} G_{\sharp}(\varphi)$ when $\varphi$ describes $\Phi_{\text {temp }}^{\text {orth }}(d, \delta)$.

Fix $\varphi \in \Phi_{\text {temp }}^{\text {orth }}(d, \delta)$. Denote by $S(\varphi)$ the centralizer in $S O(d, \mathbb{C})$ of the image of $\varphi$, and by $S(\varphi)^{0}$ its identity component. The group $S(\varphi) / S(\varphi)^{0}$ is again a product of copies of $\mathbb{Z} / 2 \mathbb{Z}$. We denote by $\Pi(\varphi)$ the disjoint union of $\Pi^{G_{i}}(\varphi)$ and $\Pi^{G_{a}}(\varphi)$. Then the conjecture says there is a bijection

$$
\begin{aligned}
\left(S(\varphi) / S(\varphi)^{0}\right)^{\wedge} & \rightarrow \Pi(\varphi) \\
\epsilon & \mapsto \pi(\varphi, \epsilon) .
\end{aligned}
$$

A great recent work of Arthur, but not yet published, proves a substantial part of the parametrization's conjectures, cf. 4.8 for more details.

1.4. Weak form of the Gross-Prasad conjecture. From now on, I assume the parametrization's conjectures as stated in Section 1.3. For a smooth irreducible representation $\pi$ of $G_{i}(F)$, resp. $G_{a}(F)$, and for a smooth irreducible representation $\sigma$ of $H_{i}(F)$, resp. $H_{a}(F)$, we define the multiplicity $m(\pi, \sigma) \in\{0,1\}$ as in Section 1.2.

TheOREM A. Let $\varphi \in \Phi_{\text {temp }}^{\text {orth }}(d, \delta)$ and $\varphi^{H} \in \Phi_{\text {temp }}^{\text {orth }}\left(d^{\prime}-1\right)$. There exists a unique pair $(\pi, \sigma) \in\left(\Pi^{G_{i}}(\varphi) \times \Pi^{H_{i}}\left(\varphi^{H}\right)\right) \sqcup\left(\Pi^{G_{a}}(\varphi) \times \Pi^{H_{a}}\left(\varphi^{H}\right)\right)$ such that $m(\pi, \sigma)=1$.

Cf. [W2], th. 7.10. It's the first assertion of the local Gross-Prasad conjecture ([GP], conjecture 10.7). This result was previously know in some special cases ([GR], [GGP1]).

1.5. Strong form of the Gross-Prasad conjecture. Let $\varphi$ and $\varphi^{H}$ as in the theorem A. This theorem defines a unique pair, which is parametrized by an element $\left(\epsilon, \epsilon^{H}\right) \in\left(S(\varphi) / S(\varphi)^{0}\right)^{\wedge} \times\left(S\left(\varphi^{H}\right) / S\left(\varphi^{H}\right)^{0}\right)^{\wedge}$. I state the following theorem in an imprecise form.

TheOREM B. The parameters $\epsilon$ and $\epsilon^{H}$ can be explicitly computed.

Cf. [W4], th. 4.9. This assertion is the second part of the local GrossPrasad conjecture. As in the global situation, we can associate to $\varphi$ a finite family $\left(\boldsymbol{\pi}_{1}, \ldots, \boldsymbol{\pi}_{k}\right)$ of smooth irreducible representations of groups $G L\left(d_{i}, F\right)$, of the discrete serie, and similarly, we can associate to $\varphi^{H}$ a finite family $\left(\boldsymbol{\sigma}_{1}, \ldots, \boldsymbol{\sigma}_{l}\right)$ of representations of groups $G L\left(d_{j}^{\prime}, F\right)$. Then $\epsilon$ and $\epsilon^{H}$ are related to the $\epsilon$-factors $\epsilon\left(\boldsymbol{\pi}_{i} \times \boldsymbol{\sigma}_{j}, 1 / 2, \psi_{F}\right)$ defined by Jacquet, Piatetski-Shapiro and Shalika. 
1.6. Generalizations. Consider parameters $\varphi \in \Phi^{\text {orth }}(d, \delta)$ and $\varphi^{H} \in$ $\Phi^{\text {orth }}\left(d^{\prime}-1\right)$ that are not tempered. Using the theorem of Langlands' quotient, we can again define $L$-packets $\Pi(\varphi)$ and $\Pi\left(\varphi^{H}\right)$, cf. Section 6.1. In general, there is no conjecture (at the present time) that computes the multiplicities $m(\pi, \sigma)$. But the local Gross-Prasad conjecture holds if $\Pi(\varphi)$ and $\Pi\left(\varphi^{H}\right)$ are generic. We will define in 6.2 what is a generic $L$-packet. This property is weaker than to be tempered. For instance, all local components of a cuspidal automorphic representation of $G L(n, \mathbb{A})$ are generic.

Theorem C (joint with Moeglin). Let $\varphi \in \Phi^{\text {orth }}(d, \delta)$ and $\varphi^{H} \in \Phi^{\text {orth }}$ $\left(d^{\prime}\right)$. Assume $\Pi(\varphi)$ and $\Pi\left(\varphi^{H}\right)$ are generic. Then the theorems $A$ and $B$ holds.

Cf. [MW], introduction.

Instead of a decomposition $V=D \oplus W$, with a line $D$, assume now there is a decomposition $V=Z \oplus W$, where $\operatorname{dim}(Z)$ is odd and the special orthogonal group of $Z$ is split. For smooth irreducible representations $\pi$ of $G(F)$ and $\sigma$ of $H(F)$, we can again define a multiplicity $m(\pi, \sigma)$, using a variant of Whittaker's models. We have again $m(\pi, \sigma)=0$ or 1 . The theorems A, B, and C holds well in this case.

We have assumed that, in the pair $(\operatorname{dim}(V), \operatorname{dim}(W))$, the greatest $d$ was even and the smallest was odd. But we can assume the greatest is odd and the smallest is even. The results are the same.

Our work concerns only special orthogonal groups. But Gan, Gross and Prasad consider other cases, cf. [GGP2]. If you are optimistic, you can hope that the same method holds in some other cases, for instance for unitary groups.

\section{An integral formula}

2.1. Multiplicities for more general situation. As before, let $F$ be a finite extension of $\mathbb{Q}_{p}$. Let $V$ be a vector space over $F$, of finite dimension $d$, and let $q$ be a non-degenerate quadratic form on $V$. We remove the assumption that $d$ is even. Assume given:

- an orthogonal decomposition $V=W \oplus Z$;

- a basis $\left\{v_{i} ; i=-r, \ldots, r\right\}$ of $Z$, for some $r \in \mathbb{N}$, and an element $\nu \in F^{\times}$such that, if $v=\sum_{i=-r, \ldots, r} x_{i} v_{i} \in Z$,

$$
q(v, v) / 2=\nu(-1)^{d} x_{0}^{2}+\sum_{i=1, \ldots, r} x_{i} x_{-i} .
$$

In particular, the dimension $2 r+1$ of $Z$ is odd. We denote by $G$ and $H$ the special orthogonal groups of $V$ and $W$. Introduce the flag of isotropic subspaces

$$
F v_{r} \subset F v_{r} \oplus F v_{r-1} \subset \cdots \subset F v_{r} \oplus \cdots \oplus F v_{1}
$$

of $V$, and the set $P$ of elements of $G$ that fix these subspaces. It is a parabolic subgroup. We denote by $U$ its unipotent radical. Fix a non-trivial 
and continuous character $\psi_{F}$ of $F$. Define a character $\xi$ of $U(F)$ by

$$
\xi(u)=\psi_{F}\left(\sum_{i=0, \ldots, r-1} q\left(u\left(v_{i}\right), v_{-i-1}\right)\right) .
$$

The group $H$ is included in a Levi component of $P$ and $\xi$ is fixed by conjugacy by elements of $H(F)$.

Now, let $\pi$, resp. $\sigma$, be an irreducible smooth representation of $G(F)$, resp. $H(F)$, in a complex space $E_{\pi}$, resp. $E_{\sigma}$. Let $\operatorname{Hom}_{H(F), \xi}\left(E_{\pi}, E_{\sigma}\right)$ be the space of linear applications $\varphi: E_{\pi} \rightarrow E_{\sigma}$ such that

$$
\varphi(\pi(h u) e)=\xi(u) \sigma(h) \varphi(e)
$$

for all $h \in H(F), u \in U(F), e \in E_{\pi}$. Denote by $m(\pi, \sigma)$ the dimension over $\mathbb{C}$ of $\operatorname{Hom}_{H(F), \xi}\left(E_{\pi}, E_{\sigma}\right)$. This dimension does not depend of the choice of $\psi_{F}$ nor of the basis $\left\{v_{i} ; i=-r, \ldots, r\right\}$.

REMARK. When $Z$ is a line, the situation is the same as in Section 1.2. When $\operatorname{dim}(W) \leq 1$, we have $H=\{1\}$, the representation $\sigma$ is the trivial representation of $\{1\}$ in $E_{\sigma}=\mathbb{C}$ and the elements of $\operatorname{Hom}_{H(F), \xi}\left(E_{\pi}, E_{\sigma}\right)$ are usual Whittaker's functionals on $E_{\pi}$.

Gan, Gross and Prasad have generalized the theorem of Aizenbud, Gourevitch, Rallis and Schiffmann to the present situation: $m(\pi, \sigma)$ is equal to 0 or 1 ([GGP2], corollary 20.4).

We fix $\pi$ and $\sigma$ for the whole Section 2 .

2.2. Tempered representations. We recall here in more details the definition of tempered representations, for a general reductive group $L$ defined over $F$. Let $\rho$ be a smooth irreducible representation of $L(F)$ in a complex space $E_{\rho}$. We have defined in 1.2 the coefficient $c_{e, \check{e}}$ for $e \in E_{\rho}$ and $\check{e} \in E_{\check{\rho}}$. Denote by $Z_{L}$ the center of $L$. We say that $\rho$ is of the discrete serie if and only if

- the central character of $\rho$ is unitary;

- for all $e$ and $\check{e}$, the absolute value $\left|c_{e, \check{e}}\right|$ is square-integrable on $L(F) / Z_{L}(F)$ (by the first condition, it is a function on this quotient).

Now consider a parabolic subgroup $Q$ of $L$ and a Levi component $M$ of $Q$, with $Q$ and $M$ defined over $F$. Let $\rho^{M}$ be a smooth irreducible representation of $M(F)$, of the discrete serie. Harish-Chandra has defined the induced representation $\operatorname{Ind} d_{Q}^{L}\left(\rho^{M}\right)$ of $L(F)$. It is not irreducible in general, but semisimple and of finite lenght. We say that the representation $\rho$ of $L(F)$ is tempered if and only if there exists $Q, M$ and $\rho^{M}$ as above such that $\rho$ is an irreducible component of $\operatorname{Ind} d_{Q}^{L}\left(\rho^{M}\right)$. In fact, the pair $\left(M, \rho^{M}\right)$ is uniquely determined by $\rho$ up to conjugacy by $L(F)$. It is not obvious, but true, that 
a representation is tempered if and only if its coefficients are of moderate growth, in some suitable sense.

2.3. Some results of Harish-Chandra. Let $\pi$ as before. Denote by $C_{c}^{\infty}(G(F))$ the space of functions $f: G(F) \rightarrow \mathbb{C}$ that are locally constant and of compact support (I will use later similar notation for other groups). Fix an Haar mesure on $G(F)$. Then $C_{c}^{\infty}(G(F))$ acts on the space $E_{\pi}$ by

$$
\pi(f) e=\int_{G(F)} f(g) \pi(g) e d g,
$$

for $f \in C_{c}^{\infty}(G(F))$ and $e \in E_{\pi}$. The operator $\pi(f)$ has finite rank, so we can define the distribution $f \mapsto \operatorname{trace}(\pi(f))$. Harish-Chandra has proved that this distribution was locally integrable, i.e. there exists a locally integrable function $\chi_{\pi}$ on $G(F)$ such that

$$
\operatorname{trace}(\pi(f))=\int_{G(F)} f(g) \chi_{\pi}(g) d g .
$$

The function $\chi_{\pi}$ is invariant by conjugacy. Let $t$ be a semi-simple element of $G(F)$. If $t$ is strongly regular (i.e. the centralizer $Z_{G}(t)$ of $t$ in $G$ is a torus), $\chi_{\pi}$ is constant in a neighbourhood of $t$. It's not true if $t$ is not strongly regular. In the general case, denote by $\mathfrak{g}_{t}$ the Lie algebra of the identity component $G_{t}=Z_{G}(t)^{0}$ of $Z_{G}(t)$. Denote by $N i l\left(\mathfrak{g}_{t}(F)\right)$ the set of nilpotent orbits in $\mathfrak{g}_{t}(F)$, for the adjoint action of $Z_{G}(t)^{0}(F)$. Let $U \in N i l\left(\mathfrak{g}_{t}(F)\right)$, fix a measure on $U$, invariant by this adjoint action. We define the nilpotent orbital integral

$$
f \mapsto J_{U}(f)=\int_{U} f(u) d u
$$

for $f \in C_{c}^{\infty}\left(\mathfrak{g}_{t}(F)\right)$. Fix a non-degenerate quadratic form $q_{\mathfrak{g}_{t}}$ on $\mathfrak{g}_{t}(F)$, invariant by the adjoint action of $G_{t}(F)$. We define a Fourier transform $f \mapsto \hat{f}$ on $C_{c}^{\infty}\left(\mathfrak{g}_{t}(F)\right)$ by the usual formula

$$
\hat{f}(X)=\int_{\mathfrak{g}_{t}(F)} \psi_{F}\left(q_{\mathfrak{g}_{t}}(X, Y)\right) f(Y) d Y .
$$

Then we have the Fourier transform of the nilpotent orbital integral $f \mapsto$ $J_{U}(\hat{f})$. Moreover, for $X \in \mathfrak{g}_{t}(F)$ near 0 , we define as usual $\exp (X) \in$ $G_{t}(F)$. Harish-Chandra proves ([HC], theorem 16.2) that there exists an open neighbourhood $\omega$ of 0 in $\mathfrak{g}_{t}(F)$ and a unique family $\left(c_{\pi, U}(t)\right)_{U \in N i l\left(\mathfrak{g}_{t}(F)\right)}$ of complex numbers such that, for $f \in C_{c}^{\infty}(\omega)$, we have the identity

$$
\int_{\omega} \chi_{\pi}(\exp (X) t) f(X) d X=\sum_{U \in N i l\left(\mathfrak{g}_{t}(F)\right)} c_{\pi, U}(t) J_{U}(\hat{f}) .
$$

Of course, we need to define precisely the measures: the measure on $\mathfrak{g}_{t}(F)$ is autodual for the Fourier transform; using the quadratic form $q_{\mathfrak{g} t}, U(F)$ appears as a symplectic variety and the measure on $U(F)$ is deduced from 
this structure. We see that the coefficients $c_{\pi, U}(t)$ are independent of the choice of the quadratic form $q_{\mathfrak{g} t}$.

2.4. Some tori. Consider the set $\mathcal{I}$ of subtori $T \subset H$, defined over $F$, not maximal in general, such that there exists an orthogonal decomposition $W=W^{\prime} \oplus W^{\prime \prime}$ satisfying the following conditions:

(1) $\operatorname{dim}\left(W^{\prime}\right)$ is even;

(2) denote by $H^{\prime}$ the special orthogonal group of $W^{\prime}$ (it's a subgroup of $H$ ); then $T \subset H^{\prime}$ and $T$ is a maximal subtorus of $H^{\prime}$;

(3) the maximal $F$-split subtorus of $T$ is $\{1\}$;

(4) if $d$ is odd, the special orthogonal group $G^{\prime \prime}$ of $W^{\prime \prime} \oplus Z$ is split over $F$; if $d$ is even, the special orthogonal group $H^{\prime \prime}$ of $W^{\prime \prime}$ is split over $F$.

Let $T \in \underline{\mathcal{T}}$ and $t \in T(F)$. If $t$ is in general position, $G_{t}=T \times G^{\prime \prime}$ and $\operatorname{Nil}\left(\mathfrak{g}_{t}(F)\right)=\operatorname{Nil}\left(\mathfrak{g}^{\prime \prime}(F)\right)$, where $\mathfrak{g}^{\prime \prime}$ is of course the Lie algebra of $G^{\prime \prime}$. The coefficient $c_{\pi, U}(t)$ is defined for all $U \in N i l\left(\mathfrak{g}^{\prime \prime}(F)\right)$. Assume $d$ odd. Then $G^{\prime \prime}$ is a split special orthogonal group in odd dimension, and it's well know that there exists a unique regular nilpotent orbit in $\mathfrak{g}^{\prime \prime}(F)$. Denote by $U_{\text {reg }}$ this orbit and let $c_{\pi}(t)=c_{\pi, U_{r e g}}(t)$. Assume now $d$ even. Then there exists several regular nilpotent orbits in $\mathfrak{g}^{\prime \prime}(F)$. But consider the subspace $Z_{0} \subset Z$ generated by the vectors $v_{i}$ for $i \neq 0$. It is a sum of hyperbolic planes. Consider the special orthogonal group $H_{0}^{\prime \prime}$ of $W^{\prime \prime} \oplus Z_{0}$. Because $H^{\prime \prime}$ is split, $H_{0}^{\prime \prime}$ is also split. For the same reason as before, there exists a unique regular nilpotent orbit in $\mathfrak{h}_{0}^{\prime \prime}(F)$. By the inclusion $H_{0}^{\prime \prime} \subset G^{\prime \prime}$, it is included in some nilpotent orbit of $\mathfrak{g}^{\prime \prime}(F)$ and in fact in a regular nilpotent orbit. We denote by $U_{\text {reg }}$ this orbit and we define $c_{\pi}(t)=c_{\pi, U_{r e g}}(t)$ as before. Then $c_{\pi}$ is a function defined almost everywhere on $T(F)$.

Similarly, we have $H_{t}=T \times H^{\prime \prime}$ and $\operatorname{Nil}\left(\mathfrak{h}_{t}(F)\right)=\operatorname{Nil}\left(\mathfrak{h}^{\prime \prime}(F)\right)$. If $d$ is even, $H^{\prime \prime}$ is split and in "odd dimension", so $\mathfrak{h}^{\prime \prime}(F)$ has a unique nilpotent regular orbit $U_{\text {reg }}^{H}$. If $d$ is odd, and $\operatorname{dim}\left(W^{\prime \prime}\right) \leq 2, \mathfrak{h}^{\prime \prime}(F)$ has again a unique nilpotent regular orbit $U_{\text {reg }}^{H}$. Assume $d$ odd and $\operatorname{dim}\left(W^{\prime \prime}\right) \geq 4$. Because $G^{\prime \prime}$ is split, we see that there exists an orthogonal decomposition $W^{\prime \prime}=W_{0} \oplus D_{0}$, where $D_{0}$ is a line and the space $D_{0} \oplus F v_{0}$ is an hyperbolic plane. As above, the Lie algebra of the special orthogonal group of $W_{0}$ has a unique regular nilpotent orbit. This orbit is included in a nilpotent orbit of $\mathfrak{h}^{\prime \prime}(F)$, denote by $U_{r e g}^{H}$ this orbit. In all cases, let $c_{\sigma}(t)=c_{\sigma, U_{r e g}^{H}}(t)$. Then $c_{\sigma}$ is a function defined almost everywhere on $T(F)$.

We define two others functions almost everywhere on $T(F)$. Let $t \in$ $T(F)$ in general position. We put

$$
\Delta(t)=\left|\operatorname{det}\left(\left.(1-t)\right|_{\mid W^{\prime}}\right)\right|_{F},
$$

where $|\cdot|_{F}$ is the usual absolute value of $F$;

$$
D^{H}(t)=\left|\operatorname{det}\left((\operatorname{ad}(t)-1) \mid \mathfrak{h}(F) / \mathfrak{h}_{t}(F)\right)\right|_{F} .
$$


And we denote by $W(H, T)$ the quotient of the normalizer of $T$ in $H(F)$ by the centralizer. It is a finite group.

2.5. The integral formula. The group $H(F)$ acts by conjugacy on $\underline{\mathcal{I}}$. We fix a set $\mathcal{T}$ of representatives of conjugacy classes. Let

$$
m_{\text {geom }}(\pi, \sigma)=\sum_{T \in \mathcal{T}}|W(H, T)|^{-1} \int_{T(F)} c_{\pi}(t) c_{\sigma}(t) D^{H}(t) \Delta(t)^{r} d t
$$

where the measures on the tori have total mass 1 .

Proposition. (i) The above formula is absolutely convergent.

(ii) Assume $\pi$ and $\sigma$ are tempered. Then $m(\pi, \sigma)=m_{\text {geom }}(\pi, \sigma)$.

Cf. [W2], théorème 7.1.

2.6. About the proof: the geometric side. The proof of the proposition is very long. I will describe the starting point only. We choose a family $\left(K_{N}\right)_{N \geq 1}$ of compact open subsets of $H(F) U(F) \backslash G(F)$ such that $K_{N} \subset K_{N+1}$ and $\bigcup_{N>1} K_{N}=H(F) U(F) \backslash G(F)$. Let $\kappa_{N}$ be the characteristic function of the inverse image of $K_{N}$ in $G(F)$. For $N \geq 1$ and $f \in C_{c}^{\infty}(G(F))$, define

$$
\begin{aligned}
J_{N}\left(\theta_{\sigma}, f\right)= & \int_{H(F) U(F) \backslash G(F)} \\
& \times \int_{H(F)} \int_{U(F)} \theta_{\sigma}(h) f\left(g^{-1} h u g\right) \xi(u)^{-1} d u d h \kappa_{N}(g) d g .
\end{aligned}
$$

This expression is absolutely convergent. We want to compute its limit when $N$ tends to $\infty$.

Here, the idea is the same as for the Arthur's local trace formula. There are two ways for the computation. The first is "geometric": we express the integral using orbital integrals or weighted orbital integrals of the function $f$. In fact, it is more complicated as for the local trace formula, because the integrals have more singularities as in this case. These singularities are reflected in the final formula by the presence of integrals over non maximal tori: these integrals appear as singularities of integrals over maximal tori. And its not possible to compute the limit of $J_{N}\left(\theta_{\sigma}, f\right)$ for all choice of the sets $K_{N}$ and all functions $f$ : we must choose particular $K_{N}$ and we must assume that $f$ is strongly cuspidal. That means that for all proper parabolic subgroup $P^{\prime}=M^{\prime} U^{\prime}$ of $G$, defined over $F$, where $M^{\prime}$ is a Levi component of $P^{\prime}$ and $U^{\prime}$ is the unipotent radical of $P^{\prime}$, we have

$$
\int_{U^{\prime}(F)} f\left(m^{\prime} u^{\prime}\right) d u^{\prime}=0
$$


for all $m^{\prime} \in M^{\prime}(F)$. Under those conditions, we can compute the limit as

$$
\lim _{N \rightarrow \infty} J_{N}\left(\theta_{\sigma}, f\right)=m_{\text {geom }}(f, \sigma)
$$

where $m_{\text {geom }}(f, \sigma)$ is similar to $m_{\text {geom }}(\pi, \sigma)$. The function $c_{\pi}$ is replaced by a function $c_{f}$ deduced from weighted orbital integrals of $f$. Cf. [W1] théorème 7.8 .

I will give a basic example of the computation. Consider the case where $\operatorname{dim}(V)=3, \operatorname{dim}(W)=2$ and $G$ and $H$ are split. We can identify $G$ with $P G L(2)$ and $H$ with its diagonal subtorus. Let $K=P G L\left(2, \mathfrak{o}_{F}\right)$ be the standard maximal subgroup of $G(F)$, where $\mathfrak{o}_{F}$ is the ring of integers of $F$. We can write every element $g \in G(F)$ as a product

$$
g=\left(\begin{array}{ll}
a & 0 \\
0 & 1
\end{array}\right)\left(\begin{array}{ll}
1 & b \\
0 & 1
\end{array}\right) k
$$

with $a \in F^{\times}, b \in F$ and $k \in K$ (we consider the two first matrices as elements of $P G L(2, F))$. The measure $d g$ decomposes as $d g=|a|_{F}^{-1} d a d b d k$. We assume that $\kappa_{N}(g)=1$ if $\operatorname{val}_{F}(b) \geq-N$ and $\kappa_{N}(g)=0$ if $\operatorname{val}_{F}(b)<-N$, where $v a l_{F}$ is the usual valuation of $F$. We can consider the character $\theta_{\sigma}$ as an usual character of $F^{\times}$. Then

$$
\begin{gathered}
J_{N}\left(\theta_{\sigma}, f\right)=\int_{K} \int_{F^{\times}} \int_{b \in F ; v_{a l}(b) \geq-N} \theta_{\sigma}(a) \\
f\left(k^{-1}\left(\begin{array}{cc}
1 & -b \\
0 & 1
\end{array}\right)\left(\begin{array}{cc}
a & 0 \\
0 & 1
\end{array}\right)\left(\begin{array}{cc}
1 & b \\
0 & 1
\end{array}\right) k\right) d b|a|_{F}^{-1} d a d k .
\end{gathered}
$$

We define $f^{K}$ by

$$
f^{K}(g)=\int_{K} f\left(k^{-1} g k\right) d k
$$

Then

$$
\begin{aligned}
J_{N}\left(\theta_{\sigma}, f\right)= & \int_{F^{\times}} \int_{b \in F ; v a l_{F}(b) \geq-N} \theta_{\sigma}(a) f^{K} \\
& \times\left(\left(\begin{array}{cc}
a & (a-1) b \\
0 & 1
\end{array}\right)\right) d b|a|_{F}^{-1} d a .
\end{aligned}
$$

What happens if we suppress the condition $\operatorname{val}_{F}(b) \geq-N$ ? Changing $b$ in $(a-1)^{-1} b$, we obtain the integral

$$
\int_{F^{\times}} \int_{F} \theta_{\sigma}(a) f^{K}\left(\left(\begin{array}{cc}
a & b \\
0 & 1
\end{array}\right)\right) d b|a|_{F}^{-1}|a-1|_{F}^{-1} d a .
$$

The support of the integrand is compact. But the integral is not convergent because the function $a \mapsto|a-1|_{F}^{-1}$ is not integrable in a neighbourhood of $a=1$ (the point $a=1$ corresponds to the point $1 \in H(F)$ ). So we choose some auxiliary constant $\epsilon>0$ and we decompose our integral (1) in the sum of an integral over the domain $|a-1|_{F}>\epsilon$ and an integral over the domain $|a-1|_{F} \leq \epsilon$. In the first integral, we can use the dominate convergence theorem: its limit when $N \rightarrow \infty$ is the integral (2), restricted to the domain 
$|a-1|_{F}>\epsilon$. In fact, the limit is 0 : the integral over $b$ is 0 because $f$ is strongly cuspidal. Consider the second integral. Because $f^{K}$ is locally constant of compact support, we can choose $\epsilon$ such that

$$
f^{K}\left(\left(\begin{array}{cc}
a & (a-1) b \\
0 & 1
\end{array}\right)\right)=f^{K}\left(\left(\begin{array}{cc}
1 & (a-1) b \\
0 & 1
\end{array}\right)\right)
$$

for every $b$ and every $a$ such that $|a-1|_{F}<\epsilon$. We can also assume that $\theta_{\sigma}(a)=1$ for every such $a$. Let $x=a-1$. The condition $|a-1|_{F}<\epsilon$ is equivalent to $\operatorname{val}_{F}(x)>n$, where $n$ is some big integer determined by $\epsilon$. Then our integral is equal to

$$
\int_{x \in F ; v^{\prime} l_{F}(x)>n} \int_{b \in F ; v^{\prime} l_{F}(b) \geq-N} f^{K}\left(\left(\begin{array}{cc}
1 & x b \\
0 & 1
\end{array}\right)\right) d b d x .
$$

We change $b$ to $b x^{-1}$ and we obtain

$$
\int_{x \in F ; \operatorname{val}_{F}(x)>n} \int_{b \in F ; \operatorname{val}_{F}(b) \geq-N+\operatorname{val}_{F}(x)} f^{K}\left(\left(\begin{array}{cc}
1 & b \\
0 & 1
\end{array}\right)\right) d b|x|_{F}^{-1} d x .
$$

Assume that we can permute the two integrals. Then we obtain

$$
\int_{b \in F} \int_{x \in F ; n<v^{2} l_{F}(x) \leq N+v a l_{F}(b)} f^{K}\left(\left(\begin{array}{cc}
1 & b \\
0 & 1
\end{array}\right)\right)|x|_{F}^{-1} d x d b .
$$

Because the support of $f^{K}$ is compact, we can assume $v a l_{F}(b) \geq c$ for some constant $c$. Then $N+v_{a l}(b) \geq n$ if $N$ is big and the inner integral is equal to $N+\operatorname{val}_{F}(b)-n$ (assuming that the measure of the set of units is 1 ). We obtain

$$
\int_{b \in F} f^{K}\left(\left(\begin{array}{ll}
1 & b \\
0 & 1
\end{array}\right)\right)\left(N-n+\operatorname{val}_{F}(b)\right) d b .
$$

This expression is absolutely convergent. If we replace $f^{K}$ by its absolute value in the preceding computation, we obtain a similar result. Then the permutation of integrals is justified and our integral is equal to (3), or to the sum of

$$
(N-n) \int_{b \in F} f^{K}\left(\left(\begin{array}{ll}
1 & b \\
0 & 1
\end{array}\right)\right) d b
$$

and

$$
\int_{b \in F} f^{K}\left(\left(\begin{array}{ll}
1 & b \\
0 & 1
\end{array}\right)\right) \operatorname{val}_{F}(b) d b .
$$

But (4) is 0 for the same reason as before: the function $f$ is strongly cuspidal. Up to a constant, the expression (5) is the unipotent weighted orbital integral of $f$ as defined by Arthur. Coming back to our general formula for $m_{\text {geom }}(f, \sigma)$, we have only one torus in $\mathcal{T}$ : the torus $T=\{1\}$. For the unique element $1 \in T(F)$, we have $c_{\sigma}(1)=1$ and $c_{f}(1)$ is the weighted orbital integral above. 
2.7. The spectral side. The second way for computing the limit $\lim _{N \rightarrow \infty} J_{N}\left(\theta_{\sigma}, f\right)$ is "spectral". We need to introduce some notations. We call "Levi subgroup of $G$ " a subgroup $L \subset G$ defined over $F$ that is a Levi component of some parabolic subgroup of $G$ defined over $F$. We fix some minimal Levi subgroup $M_{0}$ of $G$ and we denote by $\mathcal{L}\left(M_{0}\right)$ the set of Levi subgroup of $G$ that contains $M_{0}$. Let $L \in \mathcal{L}\left(M_{0}\right)$. Denote by $X^{*}(L)$ the group of algebraic characters of $L$ defined over $F$. Let $\mathcal{A}_{L}^{*}=X^{*}(L) \otimes_{\mathbb{Z}} \mathbb{R}$, $\mathcal{A}_{L, \mathbb{C}}^{*}=\mathcal{A}_{L}^{*} \otimes_{\mathbb{R}} \mathbb{C}$ and $a_{L}=\operatorname{dim}\left(\mathcal{A}_{L, \mathbb{C}}^{*}\right)$. Each element $\lambda \in \mathcal{A}_{L, \mathbb{C}}^{*}$ defines a continuous homomorphism $\lambda_{L}: L(F) \rightarrow \mathbb{C}^{\times}$as follows. If $\lambda=\sum_{i=1, \ldots, n} s_{i} \chi_{i}$, with $s_{i} \in \mathbb{C}$ and $\chi_{i} \in X^{*}(L)$, and if $l \in L(F)$, we put

$$
\lambda_{L}(l)=\prod_{i=1, \ldots, n}\left|\chi_{i}(l)\right|_{F}^{s_{i}} .
$$

Let's remark that the values $\left|\chi_{i}(l)\right|_{F}$ are all integral powers of the number of elements $q$ of the residual field of $F$. This implies that $\lambda_{L}=1$ if $s_{i} \in$ $(2 \pi i / \log (q)) \mathbb{Z}$ for all $i$. So the homomorphism $\lambda_{L}$ depends only of the image of $\lambda$ in $\mathcal{A}_{L, \mathbb{C}}^{*} / i \mathcal{A}_{L, F}^{\vee}$, where $\mathcal{A}_{L, F}^{\vee}$ is some lattice in $\mathcal{A}_{L}^{*}$. If $\rho$ is a smooth representation of $L(F)$, we denote by $\rho_{\lambda}$ the representation $l \mapsto \lambda_{L}(l) \rho(l)$. If $\rho$ is tempered, or of the discrete serie, and if $\lambda \in i \mathcal{A}_{L}^{*}$, then $\rho_{\lambda}$ is also tempered, or of the discrete serie. We denote by $\Pi_{2}(L)$ the set of irreducible representations of $L(F)$ of the discrete serie. Arthur has defined a set $\Pi_{\text {ell }}(L)$ of "elliptic" representations that are not always irreducible. It is slightly bigger as $\Pi_{2}(L)$. We can say that it is the set of virtual representations that satisfy the same condition of growth as the irreducible representations of the discrete serie. For a smooth irreducible representation $\rho$ of $L(F)$, of finite length, Arthur has defined its weighted character, that is a distribution $f \mapsto J_{L}^{G}(\rho, f)$ on $C_{c}^{\infty}(G(F))$. When $L=G$, it is the usual character $\theta_{\rho}$ and it is an invariant distribution (invariant by conjugacy by $G(F)$ ). But, if $L \subsetneq G$, the definition involves intertwining operators and the distribution is not invariant.

A Levi subgroup $L \in \mathcal{L}\left(M_{0}\right)$ is not a special orthogonal group, if $L \neq G$. But it is a product

$$
L=L_{0} \times G L\left(n_{1}\right) \times \cdots \times G L\left(n_{k}\right) .
$$

The group $L_{0}$ is the special orthogonal group of a subspace $V_{0} \subset V$, of which the orthogonal is a sum of hyperbolic planes. Let $\rho$ be an smooth irreducible representation of $L(F)$. We write $\rho=\rho_{0} \otimes\left(\otimes_{i} \rho_{i}\right)$, where $\rho_{0}$ is a representation of $L_{0}(F)$ and the $\rho_{i}$ are representations of the factors $G L\left(n_{i}\right)$. Up to conjugacy, the spaces $V_{0}$ and $W$ satisfy the same conditions as our starting spaces $V$ and $W$ (may be the roles of $V$ and $W$ are reversed). So we can define the multiplicity $m\left(\rho_{0}, \sigma\right)$ (or $m\left(\sigma, \rho_{0}\right)$ if the roles of $V$ and $W$ are reversed) and we put $m(\rho, \sigma)=m\left(\rho_{0}, \sigma\right)$ (or $\left.m\left(\sigma, \rho_{0}\right)\right)$. Here the representation $\rho$ was irreducible, but it is possible to adapt the definition to the case of an elliptic non irreducible representation. 
We come back to our expression $J_{N}\left(\theta_{\sigma}, f\right)$. Using the Plancherel formula, due to Harish-Chandra in this case, we express $f$ as an integral over the set of tempered irreducible representations of $G(F)$ of the traces of $f$ in these representations. After a long computation, which follows closely the one of Arthur (but it's also necessary to assume that $f$ is strongly cuspidal), we obtain

$$
\begin{aligned}
\lim _{N \rightarrow \infty} J_{N}\left(\theta_{\sigma}, f\right)= & \sum_{L \in \mathcal{L}\left(M_{0}\right)}\left|W^{L}\right|\left|W^{G}\right|^{-1}(-1)^{a_{L}} \sum_{\rho \in \Pi_{\text {ell }}(L) / i \mathcal{A}_{L}^{*} ; m(\rho, \sigma)=1} \\
& c(\rho) \int_{i \mathcal{A}_{L}^{*} / i \mathcal{A}_{L, F}^{\vee}} J_{L}^{G}\left(\rho_{\lambda}, f\right) d \lambda .
\end{aligned}
$$

Cf. [W2] théorème 6.1. The terms $W^{L}$ and $W^{G}$ are the usual Weyl groups of $L$ and $G$, relative to the minimal Levi $M_{0}$. The term $c(\rho)$ is some explicit constant, depending of the measures. The important point is the condition $m(\rho, \sigma)=1$ that appears in our formula. We explain this fact in 2.9.

Using the method of Arthur, we can transform the preceding formulas such that they contain only invariant distributions (and not weighted characters, that are not invariant). Then, assuming $\pi$ of the discrete serie, we choose for $f$ a pseudo-coefficient of $\pi$, that is a function such that $\chi_{\pi}(f)=1$ but $\chi_{\pi^{\prime}}(f)=0$ for all irreducible tempered representation $\pi^{\prime}$ of $G(F), \pi^{\prime} \neq \pi$. Then we obtain the second assertion of the proposition 2.5 by comparison of the two limits computed in 2.6 and above. The general case where $\pi$ is only tempered can be deduced by studying the behaviour of multiplicities by induction.

2.8. Construction of intertwining homomorphisms. A technical, but important point, is to describe explicitly the elements of the space $\operatorname{Hom}_{H(F), \xi}\left(E_{\pi}, E_{\sigma}\right)$ (that is a complex line, or $\{0\}$ ). Assume for simplicity $r=0$, i.e. $\operatorname{dim}(V)=\operatorname{dim}(W)+1$. Then $\operatorname{Hom}_{H(F), \xi}\left(E_{\pi}, E_{\sigma}\right)=$ $\operatorname{Hom}_{H(F)}\left(E_{\pi}, E_{\sigma}\right)$. For $e \in E_{\pi}$ and $\check{e} \in E_{\check{\pi}}$, define a coefficient

$$
g \mapsto<\check{e}, \pi(g) e>
$$

on $G(F)$. For $e^{\prime} \in E_{\sigma}$ and $\check{e}^{\prime} \in E_{\check{\sigma}}$, define a coefficient

$$
h \mapsto<\check{\sigma}(h) \check{e}^{\prime}, e^{\prime}>
$$

on $H(F)$. Consider the integral

$$
L_{\pi, \sigma}\left(\check{e}, e, \check{e}^{\prime}, e^{\prime}\right)=\int_{H(F)}<\check{e}, \pi(h) e><\check{\sigma}(h) \check{e}^{\prime}, e^{\prime}>d h .
$$

Assume $\pi$ and $\sigma$ are tempered. Then this integral is absolutely convergent. It defines a linear form $L_{\pi, \sigma}$ on $E_{\check{\pi}} \otimes E_{\pi} \otimes E_{\check{\sigma}} \otimes E_{\sigma}$. Assume $L_{\pi, \sigma} \neq 0$. Fix $\check{e}_{0}, e_{0}, \check{e}_{0}^{\prime}$ and $e_{0}^{\prime}$ such that $L_{\pi, \sigma}\left(\check{e}_{0}, e_{0}, \check{e}_{0}^{\prime}, e_{0}^{\prime}\right) \neq 0$. We define a linear application $l: E_{\pi} \rightarrow E_{\breve{\sigma}}^{*}$ by the formula

$$
<\check{e}^{\prime}, l(e)>=L_{\pi, \sigma}\left(\check{e}_{0}, e, \check{e}^{\prime}, e_{0}^{\prime}\right)
$$


for $e \in E_{\pi}$ and $\check{e}^{\prime} \in E_{\check{\sigma}}$. It's easy to prove that $l$ takes values in $E_{\sigma} \subset E_{\check{\sigma}}^{*}$ and $l \in \operatorname{Hom}_{H(F)}\left(E_{\pi}, E_{\sigma}\right)$. Because $L_{\pi, \sigma} \neq 0$, we have also $l \neq 0$. Therefore, $\operatorname{Hom}_{H(F)}\left(E_{\pi}, E_{\sigma}\right) \neq\{0\}$ if $L_{\pi, \sigma} \neq 0$. The converse is more difficult, but true, cf. [W2] proposition 5.7.

Proposition. Assume $\pi$ and $\sigma$ tempered. Then $L_{\pi, \sigma} \neq 0$ if and only if $\operatorname{Hom}_{H(F)}\left(E_{\pi}, E_{\sigma}\right) \neq\{0\}$.

Variant. When $\pi$ and $\sigma$ are unitary, in particular when they are tempered, we can fix positive definite hermitian products over the spaces $E_{\pi}$ and $E_{\sigma}$ (linear in the second variable and anti-linear in the first) invariant by the actions of $G(F)$ and $H(F)$, and define

$$
\mathcal{L}_{\pi, \sigma}\left(e_{1}, e_{2}, e_{1}^{\prime}, e_{2}^{\prime}\right)=\int_{H(F)}\left(e_{1}, \pi(h) e_{2}\right)\left(\sigma(h) e_{1}^{\prime}, e_{2}^{\prime}\right) d h
$$

for $e_{1}, e_{2} \in E_{\pi}$ and $e_{1}^{\prime}, e_{2}^{\prime} \in E_{\sigma}$. We have a similar result for this semi-linear form. Moreover, if we assume $\operatorname{Hom}_{H(F)}\left(E_{\pi}, E_{\sigma}\right) \neq\{0\}$ and if we fix some non-zero element $l$ in this space, we see that there exists some constant $c \neq 0$ such that

$$
\mathcal{L}_{\pi, \sigma}\left(e_{1}, e_{2}, e_{1}^{\prime}, e_{2}^{\prime}\right)=c\left(e_{1}^{\prime}, l\left(e_{2}\right)\right)\left(l\left(e_{1}\right), e_{2}^{\prime}\right) .
$$

2.9. The basic computation of the spectral side. Here we assume that $Z$ is a line as in the Section 1. The Plancherel formula express $f$ as an integral over the set of tempered irreducible representations of $G(F)$ of the traces of $f$ in these representations. Consider an irreducible representation $\rho$ of $G(F)$ of the discrete serie. The contribution of $\rho$ to this formula is the function

$$
g \mapsto d(\rho) \operatorname{trace}\left(\rho\left(g^{-1}\right) \rho(f)\right),
$$

where $d(\rho)$ is the formal degree of $\rho$ as defined later. Assume that it is the only contribution to the Plancherel formula. So $f(g)$ is equal to the expression above. Then

$$
\begin{aligned}
& J_{N}\left(\theta_{\sigma}, f\right) \\
& \quad=d(\rho) \int_{H(F) \backslash G(F)} \int_{H(F)} \theta_{\sigma}(h) \operatorname{trace}\left(\rho\left(g^{-1} h^{-1} g\right) \rho(f)\right) d h \kappa_{N}(g) d g .
\end{aligned}
$$

Fix invariant positive definite hermitian products over the spaces $E_{\rho}$ and $E_{\sigma}$ and fix orthonormal basis $\left(e_{i}\right)_{i \in I}$ of $E_{\rho}$ and $\left(e_{j}^{\prime}\right)_{j \in J}$ of $E_{\sigma}$. Fix $g \in G(F)$. For $h \in H(F)$, we have

$$
\begin{aligned}
\operatorname{trace}\left(\rho\left(g^{-1} h^{-1} g\right) \rho(f)\right) & =\sum_{i \in I}\left(e_{i}, \rho\left(g^{-1} h^{-1} g\right) \rho(f) e_{i}\right) \\
& =\sum_{i \in I}\left(\rho(g) e_{i}, \rho\left(h^{-1} g\right) \rho(f) e_{i}\right) .
\end{aligned}
$$

Because $f$ is biinvariant by some open compact subgroup, we can restrict the sum to a finite subset $I_{f} \subset I$ independent of $g$. By definition of the 
character $\theta_{\sigma}$, the inner integral of the expression (1) is equal to the trace of the operator

$$
\int_{H(F)} \operatorname{trace}\left(\rho\left(g^{-1} h^{-1} g\right) \rho(f)\right) \sigma(h) d h
$$

that is

$$
\sum_{j \in J}\left(e_{j}^{\prime},\left(\int_{H(F)} \sum_{i \in I_{f}}\left(\rho(g) e_{i}, \rho\left(h^{-1} g\right) \rho(f) e_{i}\right) \sigma(h) d h\right) e_{j}^{\prime}\right) .
$$

Fix $j \in J$. The corresponding term above is absolutely convergent and equal to

$$
\sum_{i \in I_{f}} \int_{H(F)}\left(\rho(g) e_{i}, \rho\left(h^{-1} g\right) \rho(f) e_{i}\right)\left(\sigma\left(h^{-1}\right) e_{j}^{\prime}, e_{j}^{\prime}\right) d h .
$$

Changing $h$ to $h^{-1}$, the integral above is $\mathcal{L}_{\rho, \sigma}\left(\rho(g) e_{i}, \rho(g) \rho(f) e_{i}, e_{j}^{\prime}, e_{j}^{\prime}\right)$. If $m(\rho, \sigma)=0$, the semi-linear form $\mathcal{L}_{\rho, \sigma}$ is 0 by the proposition 2.8. Our computation shows that $J_{N}\left(\theta_{\sigma}, f\right)=0$. Assume now that $m(\rho, \sigma)=1$. Fix some non-zero element $l \in \operatorname{Hom}_{H(F)}\left(E_{\rho}, E_{\sigma}\right)$ and let $c$ be the non-zero constant such that $2.8(1)$ is satisfied. Then (3) is equal to

$$
\sum_{i \in I_{f}} c\left(l\left(\rho(g) e_{i}\right), e_{j}^{\prime}\right)\left(e_{j}^{\prime}, l\left(\rho(g) \rho(f) e_{i}\right)\right)
$$

and (2) is equal to

$$
\sum_{j \in J} \sum_{i \in I_{f}} c\left(l\left(\rho(g) e_{i}\right), e_{j}^{\prime}\right)\left(e_{j}^{\prime}, l\left(\rho(g) \rho(f) e_{i}\right)\right) .
$$

Using the equality

$$
l\left(\rho(g) \rho(f) e_{i}\right)=\sum_{j \in J}\left(e_{j}^{\prime}, l\left(\rho(g) \rho(f) e_{i}\right)\right) e_{j}^{\prime},
$$

we obtain that (2) is equal to

$$
\sum_{i \in I_{f}} c\left(l\left(\rho(g) e_{i}\right), l\left(\rho(g) \rho(f) e_{i}\right)\right)
$$

Fix $e_{*} \in E_{\rho}$ and $e_{*}^{\prime} \in E_{\sigma}$ such that $\left(e_{*}^{\prime}, l\left(e_{*}\right)\right)=1$. We can multiply the expression above by $1=\left(e_{*}^{\prime}, l\left(e_{*}\right)\right)\left(l\left(e_{*}\right), e_{*}^{\prime}\right)$. Using $2.8(1)$, we have

$$
c\left(l\left(\rho(g) e_{i}\right), l\left(\rho(g) \rho(f) e_{i}\right)\right)\left(e_{*}^{\prime}, l\left(e_{*}\right)\right)=\mathcal{L}_{\rho, \sigma}\left(\rho(g) e_{i}, e_{*}, e_{*}^{\prime}, l\left(\rho(g) \rho(f) e_{i}\right)\right) .
$$

Using the definition of this term, (2) becomes equal to

$$
\sum_{i \in I_{f}}\left(l\left(e_{*}\right), e_{*}^{\prime}\right) \int_{H(F)}\left(\sigma(h) e_{*}^{\prime}, l\left(\rho(g) \rho(f) e_{i}\right)\right)\left(\rho(g) e_{i}, \rho(h) e_{*}\right) d h .
$$


Using once more 2.8(1), we have

$$
\left(l\left(e_{*}\right), e_{*}^{\prime}\right)\left(\sigma(h) e_{*}^{\prime}, l\left(\rho(g) \rho(f) e_{i}\right)\right)=c^{-1} \mathcal{L}_{\rho, \sigma}\left(e_{*}, \rho(g) \rho(f) e_{i}, \sigma(h) e_{*}^{\prime}, e_{*}^{\prime}\right) .
$$

Using the definition of this term, (2) becomes equal to

$$
c^{-1} \sum_{i \in I_{f}} \int_{H(F)}\left(\rho(g) e_{i}, \rho(h) e_{*}\right) \int_{H(F)}\left(\sigma\left(h^{\prime} h\right) e_{*}^{\prime}, e_{*}^{\prime}\right)\left(e_{*}, \rho\left(h^{\prime} g\right) \rho(f) e_{i}\right) d h^{\prime} d h .
$$

Coming back to (1), we obtain

$$
\begin{aligned}
J_{N}\left(\theta_{\sigma}, f\right)= & d(\rho) c^{-1} \int_{H(F) \backslash G(F)} \sum_{i \in I_{f}} \int_{H(F)}\left(\rho(g) e_{i}, \rho(h) e_{*}\right) \\
& \times \int_{H(F)}\left(\sigma\left(h^{\prime} h\right) e_{*}^{\prime}, e_{*}^{\prime}\right)\left(e_{*}, \rho\left(h^{\prime} g\right) \rho(f) e_{i}\right) d h^{\prime} d h \kappa_{N}(g) d g .
\end{aligned}
$$

Because $\rho$ is of the discrete serie, a tedious computation shows that this expression is absolutely convergent, even if we suppress the term $\kappa_{N}(g)$. We obtain first that $\lim _{N \rightarrow \infty} J_{N}\left(\theta_{\sigma}, f\right)$ is the same expression where $\kappa_{N}(g)$ is removed. Next, we can change $h$ to $\left(h^{\prime}\right)^{-1} h$ and the double integration over $g \in H(F) \backslash G(F)$ and $h^{\prime} \in H(F)$ to a unique integration over $g \in G(F)$. So

$$
\begin{aligned}
\lim _{N \rightarrow \infty} J_{N}\left(\theta_{\sigma}, f\right)= & d(\rho) c^{-1} \sum_{i \in I_{f}} \int_{G(F)} \int_{H(F)}\left(\rho(g) e_{i}, \rho(h) e_{*}\right) \\
& \times\left(\sigma(h) e_{*}^{\prime}, e_{*}^{\prime}\right)\left(e_{*}, \rho(g) \rho(f) e_{i}\right) d h d g \\
= & c^{-1} \sum_{i \in I_{f}} \int_{H(F)}\left(\sigma(h) e_{*}^{\prime}, e_{*}^{\prime}\right) d(\rho) \\
& \times \int_{G(F)}\left(\rho(g) e_{i}, \rho(h) e_{*}\right)\left(e_{*}, \rho(g) \rho(f) e_{i}\right) d g d h .
\end{aligned}
$$

For every $\epsilon_{1}, \epsilon_{2}, \epsilon_{3}, \epsilon_{4} \in E_{\rho}$, we have the equality

$$
d(\rho) \int_{G(F)}\left(\rho(g) \epsilon_{1}, \epsilon_{2}\right)\left(\epsilon_{3}, \rho(g) \epsilon_{4}\right) d g=\left(\epsilon_{1}, \epsilon_{4}\right)\left(\epsilon_{3}, \epsilon_{2}\right) .
$$

In fact, that is the definition of the formal degree of a representation of the discrete serie. Using this relation, we compute the inner integral of the expression (4), and we obtain

$$
\begin{aligned}
\lim _{N \rightarrow \infty} J_{N}\left(\theta_{\sigma}, f\right) & =c^{-1} \sum_{i \in I_{f}} \int_{H(F)}\left(\sigma(h) e_{*}^{\prime}, e_{*}^{\prime}\right)\left(e_{i}, \rho(f) e_{i}\right)\left(e_{*}, \rho(h) e_{*}\right) d h \\
& \left.=\sum_{i \in I_{f}}\left(e_{i}, \rho(f) e_{i}\right)\right) c^{-1} \int_{H(F)}\left(\sigma(h) e_{*}^{\prime}, e_{*}^{\prime}\right)\left(e_{*}, \rho(h) e_{*}\right) d h .
\end{aligned}
$$


The integral is again equal to $\mathcal{L}_{\rho, \sigma}\left(e_{*}, e_{*}, e_{*}^{\prime}, e_{*}^{\prime}\right)$. Using 2.8(1) and the definition of $e_{*}$ and $e_{*}^{\prime}$, it is equal to $c$. Then

$$
\left.\lim _{N \rightarrow \infty} J_{N}\left(\theta_{\sigma}, f\right)=\sum_{i \in I_{f}}\left(e_{i}, \rho(f) e_{i}\right)\right)=\operatorname{trace}(\rho(f)) .
$$

So we obtain the final formula

$$
\lim _{N \rightarrow \infty} J_{N}\left(\theta_{\sigma}, f\right)=\left\{\begin{array}{cl}
0, & \text { if } m(\rho, \sigma)=0, \\
\operatorname{trace}(\rho(f)), & \text { if } m(\rho, \sigma)=1,
\end{array}\right.
$$

in others words

$$
\lim _{N \rightarrow \infty} J_{N}\left(\theta_{\sigma}, f\right)=m(\rho, \sigma) \operatorname{trace}(\rho(f)) .
$$

It is the formula of 2.7 for our choice of $f$.

2.10. Intermission: the conjecture of Ichino and Ikeda. We interrupt our work to give the precise statement of the conjecture of Ichino and Ikeda quoted in 1.1. It shows the role of the semi-linear form introduced in Section 2.8. For this section, the ground field is $\mathbb{Q}$ again and we assume $d=\operatorname{dim}(V)=\operatorname{dim}(W)+1$ and $d$ is even. Let $\mathcal{V}$, resp. $\mathcal{W}$, be an irreducible subspace of the space of cuspidal automorphic forms on $G(\mathbb{Q}) \backslash G(\mathbb{A})$, resp. $H(\mathbb{Q}) \backslash H(\mathbb{A})$. Denote by $\pi$, resp. $\sigma$, the representation of $G(\mathbb{A})$ on $\mathcal{V}$, resp. of $H(\mathbb{A})$ on $\mathcal{W}$. We decompose $\pi$ and $\sigma$ as tensor products, in certain sense, of representations $\pi_{v}$ and $\sigma_{v}$, where $v$ describes the places of $\mathbb{Q}$. For every $v$, we denote by $E_{\pi_{v}}$ and $E_{\sigma_{v}}$ the space of smooth vectors of $\pi_{v}$ and $\sigma_{v}$ (if $v=\infty$ is the real place, we add the condition that the elements of $E_{\pi_{\infty}}$ and $E_{\sigma_{\infty}}$ are $K$-finite, a condition that we don't explain). Let $\varphi \in \mathcal{V}$ such that $\varphi \simeq \otimes_{v} e_{v}$, where $e_{v} \in E_{\pi_{v}}$ for every $v$, and similarly let $\psi \in \mathcal{W}$ such that $\psi \simeq \otimes e_{v}^{\prime}$. Fix a finite set $S$ of places of $\mathbb{Q}$, containing $\infty$, such that all the data are unramified for every $v \notin S$. That means that for $v \notin S$, we can fix maximal compact subgroups of $G\left(\mathbb{Q}_{v}\right)$ and $H\left(\mathbb{Q}_{v}\right)$ that are hyperspecial in the terminology of Bruhat and Tits and such that $e_{v}$ and $e_{v}^{\prime}$ are invariant by these subgroups.

We assume that all local components $\pi_{v}$ and $\sigma_{v}$ of $\pi$ and $\sigma$ are tempered. We associate to $\pi$ and $\sigma$ sets $\left\{\boldsymbol{\pi}_{1}, \ldots, \boldsymbol{\pi}_{k}\right\}$ and $\left\{\boldsymbol{\sigma}_{1}, \ldots, \boldsymbol{\sigma}_{l}\right\}$ as in 1.1. Their elements are unitary cuspidal automorphic representations of some groups, say $G L\left(d_{i}, \mathbb{A}\right)$ for $\boldsymbol{\pi}_{i}$ and $G L\left(d_{j}^{\prime}, \mathbb{A}\right)$ for $\boldsymbol{\sigma}_{j}$. For two such representations $\boldsymbol{\tau}$ of $G L(N, \mathbb{A})$ and $\boldsymbol{\tau}^{\prime}$ of $G L\left(N^{\prime}, \mathbb{A}\right)$, unramified outside $S$, we consider the partial $L$-function $L^{S}\left(\boldsymbol{\tau} \times \boldsymbol{\tau}^{\prime}, s\right)$, depending of a complex parameter $s$. For $\operatorname{Re}(s) \gg 0$, it is defined by an eulerian product

$$
L^{S}\left(\boldsymbol{\tau} \times \boldsymbol{\tau}^{\prime}, s\right)=\prod_{p \notin S} L_{p}\left(\boldsymbol{\tau} \times \boldsymbol{\tau}^{\prime}, s\right)
$$


For $p \notin S$, the theory of Hecke operators associates to $\pi_{p}$ and $\sigma_{p}$ complex parameters $\lambda_{1, p}, \ldots, \lambda_{N, p}$ and $\mu_{1, p}, \ldots, \mu_{N^{\prime}, p}$ and we have

$$
L_{p}\left(\boldsymbol{\tau} \times \boldsymbol{\tau}^{\prime}, s\right)=\prod_{i=1, \ldots, N ; j=1, \ldots, N^{\prime}}\left(1-\lambda_{i, p} \mu_{j, p} p^{-s}\right)^{-1} .
$$

The function $L^{S}\left(\boldsymbol{\tau} \times \boldsymbol{\tau}^{\prime}, s\right)$ is defined by meromorphic continuation on the whole complex plane. Moreover, it has no pole at $s=1 / 2$. In the case $\boldsymbol{\tau}=\boldsymbol{\tau}^{\prime}$, we can decompose this $L$-function as a product

$$
L^{S}(\boldsymbol{\tau} \times \boldsymbol{\tau}, s)=L^{S}\left(\boldsymbol{\tau}, \operatorname{Sym}^{2}, s\right) L^{S}\left(\boldsymbol{\tau}, \wedge^{2}, s\right) .
$$

The two functions are also eulerian products for $\operatorname{Re}(s) \gg 0$ and, for $p \notin S$, we have

$$
\begin{aligned}
L_{p}\left(\boldsymbol{\tau}, \text { Sym }^{2}, s\right) & =\prod_{1 \leq i \leq j \leq N}\left(1-\lambda_{i, p} \lambda_{j, p} p^{-s}\right)^{-1}, \\
L_{p}\left(\boldsymbol{\tau}, \wedge^{2}, s\right) & =\prod_{1 \leq i<j \leq N}\left(1-\lambda_{i, p} \lambda_{j, p} p^{-s}\right)^{-1} .
\end{aligned}
$$

The two functions have also meromorphic continuation to the whole complex plane. Put

$$
\begin{aligned}
& L^{S}(\pi \times \sigma, s)=\prod_{i=1, \ldots, k ; j=1, \ldots, l} L^{S}\left(\boldsymbol{\pi}_{i} \times \boldsymbol{\sigma}_{j}, s\right), \\
& L^{S}(\pi, A d, s)=\left(\prod_{1 \leq i<i^{\prime} \leq k} L^{S}\left(\boldsymbol{\pi}_{i} \times \boldsymbol{\pi}_{i^{\prime}}, s\right)\right)\left(\prod_{i=1, \ldots, k} L^{S}\left(\boldsymbol{\pi}_{i}, \wedge^{2}, s\right)\right), \\
& L^{S}(\sigma, A d, s)=\left(\prod_{1 \leq j<j^{\prime} \leq l} L^{S}\left(\boldsymbol{\sigma}_{j} \times \boldsymbol{\sigma}_{j^{\prime}}, s\right)\right)\left(\prod_{j=1, \ldots, l} L^{S}\left(\boldsymbol{\sigma}_{i}, S y m^{2}, s\right)\right) .
\end{aligned}
$$

In the forthcoming paper $[\mathrm{A}]$, Arthur will prove that the two last functions have no poles at $s=1$ (and it is know they have no zero at this point). Put

$$
P^{S}(\pi \times \sigma, s)=\frac{L^{S}(\pi \times \sigma, s)}{L^{S}(\pi, A d, s+1 / 2) L^{S}(\sigma, A d, s+1 / 2)} .
$$

This function is holomorphic at $s=1 / 2$.

For $v \in S$, we fix some invariant positive hermitian products on the spaces of $E_{\pi_{v}}$ and $E_{\sigma_{v}}$ and we define the semi-linear form $\mathcal{L}_{\pi_{v}, \sigma_{v}}$ as in Section 2.8 (in this section, $v$ was finite, but the definition is the same if $v=\infty$ ).

We define the integral $J(\psi, \varphi)$ as in 1.1 , and we denote by $\|\varphi\|$ and $\|\psi\|$ the $L^{2}$-norm of $\varphi$ and $\psi$. For these definitions, we use the Tamagawa measures on $G(\mathbb{Q}) \backslash G(\mathbb{A})$ and $H(\mathbb{Q}) \backslash H(\mathbb{A})$. Remark that, for $v \in S$, a measure on $H\left(\mathbb{Q}_{v}\right)$ is used to define the form $\mathcal{L}_{\pi_{v}, \sigma_{v}}$. We assume that the Tamagawa measure is equal to the product of these measures for $v \in S$ and 
of the "unramified" measures for $v \notin S$ (these measures gives the measure 1 to an hyperspecial compact subgroup). Let

$$
\Delta^{S}=\zeta^{S}(2) \ldots \zeta^{S}(d-2) L^{S}(\delta, d / 2),
$$

where $\zeta^{S}(s)$ is the partial Riemann's $\zeta$-function and $L^{S}(\delta, s)$ is the partial Dirichlet's function associated to the quadratic character determined by $\delta$. At last, let

$$
b= \begin{cases}k+l, & \text { if all } d_{i} \text { are even, } \\ k+l-1, & \text { if at least one } d_{i} \text { is odd. }\end{cases}
$$

Then Ichino and Ikeda conjecture the following equality

$$
\frac{|J(\psi, \varphi)|^{2}}{\|\varphi\|^{2}\|\psi\|^{2}}=2^{-b} \Delta^{S} P^{S}(\pi \times \sigma, 1 / 2) \prod_{v \in S} \frac{\mathcal{L}_{\pi_{v}, \sigma_{v}}\left(e_{v}, e_{v}, e_{v}^{\prime}, e_{v}^{\prime}\right)}{\left(e_{v}, e_{v}\right)\left(e_{v}^{\prime}, e_{v}^{\prime}\right)} .
$$

The right member does not depend on the choice of $S$.

\section{An integral formula for $\epsilon$-factors}

3.1. The twisted group $G L(\mathbf{d})$. We come back to a local $p$-adic ground field $F$. We consider the group $G L(\mathbf{d})$ over $F$, where $\mathbf{d} \in \mathbb{N}$. Let $J_{\mathbf{d}}$ be the antidiagonal matrix

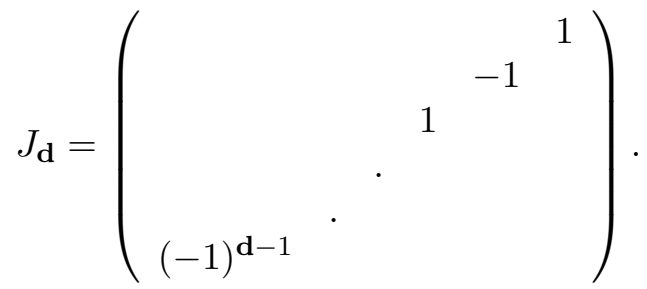

Let $\theta_{\mathbf{d}}$ be the automorphism $g \mapsto J_{\mathbf{d}} g^{-1} J_{\mathbf{d}}^{-1}$ of $G L(\mathbf{d})$. We introduce the semi-direct product $G L(\mathbf{d})^{+}=G L(\mathbf{d}) \rtimes\left\{1, \theta_{\mathbf{d}}\right\}$ and its connected component $G L(\mathbf{d}) \theta_{\mathbf{d}}$. We call this component a twisted space over $G L(\mathbf{d})$. As we will see later, the theory of twisted endoscopy relates the harmonic analysis on classical groups, in particular on special orthogonal groups, with the harmonic analysis on such twisted spaces $G L(\mathbf{d}) \theta_{\mathbf{d}}$. It is useful to describe $G L(\mathbf{d}) \theta_{\mathbf{d}}$ in terms of elementary linear algebra. Fix a vector space $\mathbf{V}$ over $F$ of dimension d, with a basis $\left(v_{i}\right)_{i=1, \ldots, \mathbf{d}}$. We identify as usual $G L(\mathbf{d})$ with the group $\mathbf{G}$ of automorphims of $\mathbf{V}$. Define a bilinear form $\boldsymbol{\theta}_{\mathbf{d}}$ on $\mathbf{V}$ by

$$
\boldsymbol{\theta}_{\mathbf{d}}\left(\sum_{i=1, . ., \mathbf{d}} x_{i} v_{i}, \sum_{i=1, \ldots, \mathbf{d}} y_{i} v_{i}\right)=(-1)^{[(d+1) / 2]} \sum_{i=1, \ldots, \mathbf{d}}(-1)^{i} x_{\mathbf{d}+1-i} y_{i} .
$$

Let's remark that $\boldsymbol{\theta}_{\mathbf{d}}$ is symmetric if $\mathbf{d}$ is odd and anti-symmetric if $\mathbf{d}$ is even. For $g \in G L(\mathbf{d})$, define the bilinear form $g \boldsymbol{\theta}_{\mathbf{d}}$ on $\mathbf{V}$ by

$$
\left(g \boldsymbol{\theta}_{\mathbf{d}}\right)\left(v, v^{\prime}\right)=\boldsymbol{\theta}_{\mathbf{d}}\left(g^{-1} v, v^{\prime}\right)
$$

for every $v, v^{\prime} \in \mathbf{V}$. The application $g \theta_{\mathbf{d}} \mapsto g \boldsymbol{\theta}_{\mathbf{d}}$ is a bijection between $G L(\mathbf{d}) \theta_{\mathbf{d}}$ and the set $\tilde{\mathbf{G}}$ of non-degenerate bilinear forms on $\mathbf{V}$. The right 
and left actions of $G L(\mathbf{d})$ on $G L(\mathbf{d}) \theta_{\mathbf{d}}$ become the actions of $\mathbf{G}$ on this set $\tilde{\mathbf{G}}$ of bilinear forms given by the formula

$$
\left(g \gamma g^{\prime}\right)\left(v, v^{\prime}\right)=\gamma\left(g^{-1} v, g^{\prime} v\right)
$$

for every bilinear form $\gamma$, every $g, g^{\prime} \in \mathbf{G}$ and every $v, v^{\prime} \in \mathbf{V}$.

Each $\gamma \in \tilde{\mathbf{G}}$ defines an automorphism $\theta_{\gamma}$ of $\mathbf{G}$ : for $g \in \mathbf{G}, \theta_{\gamma}(g)$ is the unique element of $\mathbf{G}$ such that $\theta_{\gamma}(g) \gamma=\gamma g$. Let $\mathbf{T}$ be a maximal torus of $\mathbf{G}$ defined over $F$ and let $\mathbf{B}$ be a Borel subgroup containing $\mathbf{T}$ but not necessarily defined over $F$. Let $\tilde{\mathbf{T}}$ the set of $\gamma \in \tilde{\mathbf{G}}$ such that $\theta_{\gamma}$ preserves $\mathbf{T}$ and $\mathbf{B}$. It is a principal homogeneous space over $\mathbf{T}$ (for the left or right action), in particular $\mathbf{T}$ is well determined by $\tilde{\mathbf{T}}$. For $\gamma \in \tilde{\mathbf{T}}$, the restriction of $\theta_{\gamma}$ to $\mathbf{T}$ does not depend of $\gamma$. We denote it by $\theta_{\tilde{\mathbf{T}}}$. By definition, a maximal twisted subtorus of $\tilde{\mathbf{G}}$ is such set $\tilde{\mathbf{T}}$ satisfying the condition $\tilde{\mathbf{T}}(F) \neq \emptyset$. Let $A_{\tilde{\mathbf{T}}}$ the maximal subtorus split over $F$ and contained in the subset of elements of $\mathbf{T}$ fixed by $\theta_{\tilde{\mathbf{T}}}$. We say that $\tilde{\mathbf{T}}$ is anisotropic if $A_{\tilde{\mathbf{T}}}=\{1\}$.

3.2. Embedding of twisted spaces. Let $\mathbf{d}$ and $\mathbf{r}$ be two natural integers such that $2 \mathbf{r}+1 \leq \mathbf{d}$. Let $\mathbf{V}$ be a vector space over $F$ of dimension $\mathbf{d}$ and let $\mathbf{V}=\mathbf{W} \oplus \mathbf{Z}$ be a direct decomposition, where $\operatorname{dim}(\mathbf{Z})=2 \mathbf{r}+1$. Denote $\mathbf{G}$, resp. $\mathbf{H}$, the group of linear automorphisms of $\mathbf{V}$, resp. $\mathbf{W}$, and denote $\tilde{\mathbf{G}}$, resp. $\tilde{\mathbf{H}}$, the set of non-degenerate bilinear forms on $\mathbf{V}$, resp. $\mathbf{W}$. There is an embedding of $\mathbf{H}$ in $\mathbf{G}$ : we extend every automorphism of $\mathbf{W}$ as an automorphism of $\mathbf{V}$ that acts by identity on $\mathbf{Z}$. Fix $\boldsymbol{\nu} \in F^{\times}$and a quadratic form $\zeta_{\mathbf{Z}}$ on $\mathbf{Z}$ which is a direct sum of hyperbolic spaces and of the one dimensional form

$$
(x, y) \mapsto \boldsymbol{\nu} x y .
$$

Then there is an embedding of $\tilde{\mathbf{H}}$ in $\tilde{\mathbf{G}}$ : we extend each bilinear form on $\mathbf{W}$ as the direct sum of this form on $\mathbf{W}$ and the quadratic form $\zeta_{\mathbf{Z}}$ on $\mathbf{Z}$. The two embeddings are compatible with the actions of $\mathbf{G}$ and $\mathbf{H}$ on $\tilde{\mathbf{G}}$ and $\tilde{\mathbf{H}}$.

3.3. The integral formula for twisted spaces. Let $\boldsymbol{\pi}$ be a smooth irreducible representation of $G L_{\mathbf{d}}(F)$. Assume that $\boldsymbol{\pi} \circ \theta_{\mathbf{d}} \simeq \boldsymbol{\pi}$ (we say $\boldsymbol{\pi}$ is autodual). Then we can extend $\boldsymbol{\pi}$ in a representation $\boldsymbol{\pi}^{+}$of $G L_{\mathbf{d}}^{+}(F)$. There is two possible extensions and we must choose one. For that, assume that $\boldsymbol{\pi}$ is generic. That means that there exists a non-zero linear form $\phi$ on the space $E_{\boldsymbol{\pi}}$ of $\boldsymbol{\pi}$ such that

$$
\phi(\boldsymbol{\pi}(u) e)=\psi_{F}\left(\sum_{i=1, \ldots, \mathbf{d}-1} u_{i, i+1}\right) \phi(e)
$$

for every $e \in E_{\boldsymbol{\pi}}$ and every unipotent matrix

$$
u=\left(\begin{array}{ccccc}
1 & u_{12} & u_{13} & \cdot & . \\
0 & 1 & u_{23} & \cdot & \cdot \\
\cdot & \cdot & \cdot & \cdot & \cdot \\
\cdot & \cdot & \cdot & \cdot & \cdot \\
\cdot & \cdot & 0 & 0 & 1
\end{array}\right)
$$


Such $\phi$ is called a Whittaker's functional. As it is well know, the space of Whittaker's functionals is a line. For each of the two extensions $\boldsymbol{\pi}^{+}$, the operator $\boldsymbol{\pi}^{+}\left(\theta_{\mathbf{d}}\right)$ preserves this line and acts on it by a sign \pm 1 . We choose $\boldsymbol{\pi}^{+}$such that the sign is +1 . Let $\tilde{\boldsymbol{\pi}}$ be the restriction of $\boldsymbol{\pi}^{+}$to $G L_{\mathbf{d}}(F) \theta_{\mathbf{d}}$.

Similarly, let $\boldsymbol{\sigma}$ be a smooth irreducible representation of $G L_{\mathbf{d}^{\prime}}(F)$, where $\mathbf{d}^{\prime}=\mathbf{d}-2 \mathbf{r}-1$. We assume that $\boldsymbol{\sigma} \circ \theta_{\mathbf{d}^{\prime}} \simeq \boldsymbol{\sigma}$ and that $\boldsymbol{\sigma}$ is generic. Then we define a representation $\boldsymbol{\sigma}^{+}$of $\mathbf{G}_{\mathbf{d}^{\prime}}^{+}(F)$ and its restriction $\tilde{\boldsymbol{\sigma}}$ to $G L_{\mathbf{d}^{\prime}}(F) \theta_{\mathbf{d}^{\prime}}$.

By a choice of basis of $\mathbf{V}$ and $\mathbf{W}$, we identify $G L_{\mathbf{d}}(F) \theta_{\mathbf{d}}$ with $\tilde{\mathbf{G}}(F)$ and $G L_{\mathbf{d}^{\prime}}(F) \theta_{\mathbf{d}^{\prime}}$ with $\tilde{\mathbf{H}}(F)$.

Consider data $\left(\mathbf{W}^{\prime}, \mathbf{W}^{\prime \prime}, \tilde{\mathbf{T}}^{\prime}, \zeta \mathbf{W}^{\prime \prime}\right)$ where:

- $\mathbf{W}^{\prime}$ and $\mathbf{W}^{\prime \prime}$ are supplementary subspaces of $\mathbf{W}$;

- denote by $\mathbf{H}^{\prime}$ the group of automorphisms of $\mathbf{W}^{\prime}$ and by $\tilde{\mathbf{H}}^{\prime}$ the set of non-degenerate bilinear forms on $\mathbf{W}^{\prime}$; then $\tilde{\mathbf{T}}^{\prime}$ is a maximal twisted subtorus of $\tilde{\mathbf{H}}^{\prime}$;

- $\zeta \mathbf{W}^{\prime \prime}$ is a non-degenerate quadratic form on $\mathbf{W}^{\prime \prime}$.

We impose to those data the following conditions:

(1) the dimension of $\mathbf{W}^{\prime}$ is even;

(2) the torus $\tilde{\mathbf{T}}^{\prime}$ is anisotropic;

(3) the special orthogonal group $S O\left(\zeta_{\mathbf{w}^{\prime \prime}}\right)$ of the quadratic form $\zeta \mathbf{w}^{\prime \prime}$ is quasi-split over $F$ and the special orthogonal $S O\left(\zeta_{\mathbf{v}^{\prime \prime}}\right)$ of the quadratic form $\zeta_{\mathbf{V}^{\prime \prime}}=\zeta \mathbf{W}^{\prime \prime} \oplus \zeta_{\mathbf{Z}}$ on $\mathbf{V}^{\prime \prime}=\mathbf{W}^{\prime \prime} \oplus \mathbf{Z}$ is also quasisplit.

Under those assumptions, we denote by $\tilde{\mathbf{T}}$ the subset of $\tilde{\mathbf{H}}$ whose elements are direct sums of $\zeta \mathbf{W}^{\prime \prime}$ on $\mathbf{W}^{\prime \prime}$ and a bilinear form on $\mathbf{W}^{\prime}$ which belongs to $\tilde{\mathbf{T}}^{\prime}$. This set is like a non-maximal twisted subtorus of $\tilde{\mathbf{H}}$.

Denote by $\mathbf{T}$ the maximal subtorus of $\mathbf{H}^{\prime}$ associated to $\tilde{\mathbf{T}}^{\prime}$. The group $\mathbf{T}(F)$ acts by conjugacy on $\tilde{\mathbf{T}}(F)$. We denote by $\tilde{\mathbf{T}}(F)_{/ \theta}$ the set of conjugacy classes. Denote by $\mathbf{T}_{\theta}$ the identity component of the subset of elements of $\mathbf{T}$ fixed by $\theta_{\tilde{\mathbf{T}}^{\prime}}$. Because of (2), it is an anisotropic torus and we normalize an Haar measure on $\mathbf{T}_{\theta}(F)$ by $\operatorname{mes}\left(\mathbf{T}_{\theta}(F)\right)=1$. Let $\gamma \in \tilde{\mathbf{T}}(F)_{/ \theta}$. The application

$$
\begin{aligned}
\mathbf{T}_{\theta}(F) & \rightarrow \tilde{\mathbf{T}}(F) / \theta \\
t & \mapsto t \gamma
\end{aligned}
$$

is a local isomorphism near $1 \in \mathbf{T}_{\theta}(F)$. We define the mesure on $\tilde{\mathbf{T}}(F)_{/ \theta}$ such that it is invariant by translations by $\mathbf{T}(F)$ and that the application above preserves the measures near $1 \in \mathbf{T}_{\theta}(F)$.

The normalizer $\operatorname{Norm}_{\mathbf{H}}(\tilde{\mathbf{T}})$ of $\tilde{\mathbf{T}}$ in $\mathbf{H}$ contains $\mathbf{T} \times S O\left(\zeta \mathbf{w}^{\prime \prime}\right)$. Let

$$
W(H, \tilde{\mathbf{T}})=\operatorname{Norm}_{\mathbf{H}}(\tilde{\mathbf{T}})(F) /\left(\mathbf{T}(F) \times S O\left(\zeta_{\mathbf{W}^{\prime \prime}}\right)(F)\right) .
$$

It is a finite group.

Now we define some functions almost everywhere on $\tilde{\mathbf{T}}(F)$. Let $\gamma \in$ $\tilde{\mathbf{T}}(F)$ in general position. The connected centralizer of $\gamma$ in $\mathbf{G}$ is the group $\mathbf{T}_{\theta} \times S O\left(\zeta_{\mathbf{V}^{\prime \prime}}\right)$. Thanks to Clozel, the representations of non-connected 
groups have the same properties as in the connected case. In particular, they have characters and their characters have near a semi-simple point a development as in 2.3. Then we can define $c_{\tilde{\boldsymbol{\pi}}}(\gamma)$ as we have defined $c_{\pi}(t)$ in 2.4. We define similarly $c_{\tilde{\boldsymbol{\sigma}}}(\gamma)$. Let ${ }^{t} \gamma$ the bilinear form deduced by symmetry from $\gamma:{ }^{t} \gamma\left(v, v^{\prime}\right)=\gamma\left(v^{\prime}, v\right)$. Restrict the two forms $\gamma$ and ${ }^{t} \gamma$ to $\mathbf{W}^{\prime}$. Consider the quotient

$$
\left|\operatorname{det}\left(\gamma_{\mid \mathbf{W}^{\prime}}-{ }^{t} \gamma_{\mid \mathbf{W}^{\prime}}\right)\right|_{F}\left|\operatorname{det}\left(\gamma_{\mid \mathbf{W}^{\prime}}\right)\right|_{F}^{-1}
$$

where the determinants are computed respectively to some fixed basis of $\mathbf{W}^{\prime}$. It does not depend of the basis and is non-zero for $\gamma$ in general position. We let

$$
\Delta_{\mathbf{r}}(\gamma)=|2|_{F}^{\mathbf{r}^{2}+\mathbf{r}+\mathbf{r} \operatorname{dim}\left(\mathbf{W}^{\prime \prime}\right)}\left|\operatorname{det}\left(\gamma_{\mid \mathbf{W}^{\prime}}-{ }^{t} \gamma_{\mid \mathbf{W}^{\prime}}\right)\right|_{F}^{\mathbf{r}}\left|\operatorname{det}\left(\gamma_{\mid \mathbf{W}^{\prime}}\right)\right|_{F}^{-\mathbf{r}}
$$

At last, let

$$
D^{\tilde{\mathbf{H}}}(\gamma)=\left|\operatorname{det}\left(\left(1-\theta_{\gamma}\right)_{\mid \mathfrak{h} / \mathfrak{h}_{\gamma}}\right)\right|_{F},
$$

where $\mathfrak{h}$ is the Lie algebra of $\mathbf{H}$ and $\mathfrak{h}_{\gamma}$ is the Lie algebra of the centralizer of $\gamma$ in $\mathbf{H}$.

Consider the set of subsets $\tilde{\mathbf{T}}$ obtained by the procedure above. The group $\mathbf{H}(F)$ acts by conjugacy on this set. Let $\mathcal{T}$ a set of representatives of conjugacy classes. Now we can define

$$
\epsilon_{\text {geom }}(\tilde{\boldsymbol{\pi}}, \tilde{\boldsymbol{\sigma}})=\sum_{\tilde{\mathbf{T}} \in \mathcal{T}}|W(\mathbf{H}, \tilde{\mathbf{T}})|^{-1} \int_{\tilde{\mathbf{T}}(F) / \theta} c_{\tilde{\boldsymbol{\pi}}}(\gamma) c_{\tilde{\boldsymbol{\sigma}}}(\gamma) D^{\tilde{\mathbf{H}}}(\gamma) \Delta_{\mathbf{r}}(\gamma) d \gamma
$$

3.4. Computation of some value of $\epsilon$-factors by an integral formula. Recall that Jacquet, Piatetski-Shapiro and Shalika have defined an $L$-function $L(\boldsymbol{\pi} \times \boldsymbol{\sigma}, s)$ and an $\epsilon$-factor $\epsilon\left(\boldsymbol{\pi} \times \boldsymbol{\sigma}, s, \psi_{F}\right)$, where $s$ is a complex parameter ([JPSS] theorem 2.7). Recall also that $\boldsymbol{\pi}$ and $\boldsymbol{\sigma}$ have central characters $\omega_{\boldsymbol{\pi}}$ and $\omega_{\boldsymbol{\sigma}}$. They are quadratic characters because we have assumed $\boldsymbol{\pi}$ and $\boldsymbol{\sigma}$ autodual.

Proposition. Assume $\boldsymbol{\pi}$ and $\boldsymbol{\sigma}$ autodual and tempered. Then there is an equality

$$
\omega_{\boldsymbol{\pi}}\left(\boldsymbol{\nu}(-1)^{\left[\mathbf{d}^{\prime} / 2\right]}\right) \omega_{\boldsymbol{\sigma}}\left(\boldsymbol{\nu}(-1)^{1+[\mathbf{d} / 2]}\right) \epsilon\left(\boldsymbol{\pi} \times \boldsymbol{\sigma}, 1 / 2, \psi_{F}\right)=\epsilon_{\text {geom }}(\tilde{\boldsymbol{\pi}}, \tilde{\boldsymbol{\sigma}}) .
$$

Cf. [W3] théorème 7.1.

REMARKs. (1) Because $\boldsymbol{\pi}$ and $\boldsymbol{\sigma}$ are tempered, they are also generic and we can normalize $\tilde{\boldsymbol{\pi}}$ and $\tilde{\boldsymbol{\sigma}}$ as in 3.3.

(2) The terms $\boldsymbol{\nu}$ and $\psi_{F}$ appear explicitely in the left member. But the right member depends also of $\boldsymbol{\nu}$ and $\psi_{F}: \tilde{\boldsymbol{\pi}}$ and $\tilde{\boldsymbol{\sigma}}$ are normalized using Whittaker's functionals, and this notion depends on $\psi_{F}$; and $\boldsymbol{\nu}$ is used to define the embedding $\tilde{\mathbf{H}} \rightarrow \tilde{\mathbf{G}}$.

(3) At the present time, there is a gap in the proof. We use some results coming from the twisted local trace formula and this formula is not yet 
written in the litterature. We have some reasons to think that it will be proved soon.

The proof follows the same lines as that of proposition 2.5. I want explain only why appears the $\epsilon$-factor. I assume here $\mathbf{d}^{\prime}=\mathbf{d}-1$. As for special orthogonal groups, the space $\operatorname{Hom}_{\mathbf{H}(F)}\left(E_{\boldsymbol{\pi}}, E_{\boldsymbol{\sigma}}\right)$ plays a crucial role. Aizenbud, Gourevitch, Rallis and Shiffmann have proved that its dimension is not greater as 1 . We see below that it is equal to 1 . Assuming this result, let $l$ be a non-zero element of this space and let $\gamma$ be an element of $\tilde{\mathbf{H}}(F)$. The linear form $\tilde{\boldsymbol{\sigma}}(\gamma)^{-1} \circ l \circ \tilde{\boldsymbol{\pi}}(\gamma)$ does not depend of $\gamma$. In fact, if we replace $\gamma$ by $\gamma^{\prime}=h \gamma$, with $h \in \mathbf{H}(F)$, we have

$$
\begin{aligned}
\tilde{\boldsymbol{\sigma}}\left(\gamma^{\prime}\right)^{-1} \circ l \circ \tilde{\boldsymbol{\pi}}\left(\gamma^{\prime}\right) & =\tilde{\boldsymbol{\sigma}}(\gamma)^{-1} \circ \boldsymbol{\sigma}(h)^{-1} \circ l \circ \boldsymbol{\pi}(h) \circ \tilde{\boldsymbol{\pi}}(\gamma) \\
& =\tilde{\boldsymbol{\sigma}}(\gamma)^{-1} \circ l \circ \tilde{\boldsymbol{\pi}}(\gamma),
\end{aligned}
$$

because $\boldsymbol{\sigma}(h)^{-1} \circ l \circ \boldsymbol{\pi}(h)=l$. It is easy to see that $\tilde{\boldsymbol{\sigma}}(\gamma)^{-1} \circ l \circ \tilde{\boldsymbol{\pi}}(\gamma)$ is also an element of $\operatorname{Hom}_{\mathbf{H}(F)}\left(E_{\boldsymbol{\pi}}, E_{\boldsymbol{\sigma}}\right)$. So there exists $c \in \mathbb{C}^{\times}$, independant of $\gamma$, such that

$$
\tilde{\boldsymbol{\sigma}}(\gamma)^{-1} \circ l \circ \tilde{\boldsymbol{\pi}}(\gamma)=c l
$$

A computation similar as that of 2.9 holds, where this constant $c$ plays the same role as the multiplicity $m(\rho, \sigma)$ before. But, as we see below, the constant $c$ is equal to $\epsilon\left(\boldsymbol{\pi} \times \boldsymbol{\sigma}, 1 / 2, \psi_{F}\right)$, up to elementary terms. To see that, we must recall some results of Jacquet, Piatetski-Shapiro and Shalika. At this point, it is more clear to denote $\iota: \mathbf{H} \rightarrow \mathbf{G}$ and $\tilde{\iota}: \tilde{\mathbf{H}} \rightarrow \tilde{\mathbf{G}}$ the two embeddings of 3.2. Fix a basis $\left(v_{i}\right)_{i=1, \ldots, \mathbf{d}}$ of $\mathbf{V}$ such that $\left(v_{i}\right)_{i=1, \ldots, \mathbf{d}-1}$ is a basis of $\mathbf{W}$ and $v_{\mathbf{d}}$ is a basis of $\mathbf{Z}$. Using those basis, we identify $\mathbf{G}$ with $G L(\mathbf{d})$ and $\mathbf{H}$ with $G L(\mathbf{d}-1)$. The embedding $\iota$ is

$$
\iota: h \mapsto\left(\begin{array}{cc}
h & 0 \\
0 & 1
\end{array}\right) .
$$

Fix a Whittaker's functional $\phi \neq 0$ on $E_{\boldsymbol{\pi}}$. For $e \in E_{\boldsymbol{\pi}}$, we define a function $W_{e}$ on $G L(\mathbf{d}, F)$ by

$$
W_{e}(g)=\phi(\boldsymbol{\pi}(g) e) .
$$

Similarly, we fix a non-zero Whittaker's functional on $E_{\boldsymbol{\sigma}}$ and, for $e^{\prime} \in E_{\boldsymbol{\sigma}}$, we define a function $W_{e^{\prime}}$ on $G L(\mathbf{d}-1, F)$. For $s \in \mathbb{C}$, let

$$
\mathcal{L}\left(W_{e^{\prime}}, W_{e}, s\right)=\int_{G L(\mathbf{d}-1, F)} \overline{W_{e^{\prime}}(h)} W_{e}(\iota(h))|\operatorname{det}(h)|_{F}^{s-1 / 2} d h .
$$

Jacquet, Piatetski-Shapiro and Shalika have proved the three following properties ([JPSS], theorem 2.7):

(4) this integral is absolutely convergent for $\operatorname{Re}(s)>0$ and can be extended as a meromorphic function of $s$ on the whole complex plane;

(5) we can choose $e$ and $e^{\prime}$ such that $\mathcal{L}\left(W_{e^{\prime}}, W_{e}, 1 / 2\right) \neq 0$; 
(6) let $w_{\mathbf{d}}$ be the antidiagonal matrix of rank $\mathbf{d}$ with coefficients 1 on the antidiagonal and define

$$
\check{W}_{e}(g)=W_{e}\left(w_{\mathbf{d}}^{t} g^{-1}\right) ;
$$

define similarly the function $\check{W}_{e^{\prime}}$; then we have the functional equation

$$
\begin{aligned}
\mathcal{L} & \left(\check{W}_{e^{\prime}}, \check{W}_{e}, 1-s\right) / L(1-s, \boldsymbol{\pi} \times \boldsymbol{\sigma}) \\
\quad & =\omega_{\boldsymbol{\sigma}}(-1)^{\mathbf{d}-1} \epsilon\left(\boldsymbol{\pi} \times \boldsymbol{\sigma}, s, \psi_{F}\right) \mathcal{L}\left(W_{e^{\prime}}, W_{e}, s\right) / L(s, \boldsymbol{\pi} \times \boldsymbol{\sigma})
\end{aligned}
$$

(here, we use the fact that $\boldsymbol{\pi}$ and $\boldsymbol{\sigma}$ are unitary and autodual).

Now, fix an invariant definite-positive hermitian form (.,.) on $E_{\boldsymbol{\sigma}}$. We can define a linear application $l: E_{\boldsymbol{\pi}} \rightarrow E_{\boldsymbol{\sigma}}$ by

$$
\left(e^{\prime}, l(e)\right)=\mathcal{L}\left(W_{e^{\prime}}, W_{e}, 1 / 2\right) .
$$

We see that $l \in \operatorname{Hom}_{\mathbf{H}(F)}\left(E_{\boldsymbol{\pi}}, E_{\boldsymbol{\sigma}}\right)$. Then the property (5) shows that this space is non-zero. To compute the constant $c$, we can choose $\gamma=\boldsymbol{\theta}_{\mathbf{d}-1}$, so that

$$
\tilde{\boldsymbol{\sigma}}\left(\boldsymbol{\theta}_{\mathbf{d}-1}\right)^{-1} \circ l \circ \tilde{\boldsymbol{\pi}}\left(\tilde{\iota}\left(\boldsymbol{\theta}_{\mathbf{d}-1}\right)\right)=c l .
$$

This relation is equivalent to

$$
\left(e_{1}^{\prime}, l\left(e_{1}\right)\right)=c\left(e^{\prime}, l(e)\right),
$$

where

$$
e_{1}^{\prime}=\tilde{\boldsymbol{\sigma}}\left(\boldsymbol{\theta}_{\mathbf{d}-1}\right) e^{\prime}
$$

and

$$
e_{1}=\tilde{\boldsymbol{\pi}}\left(\tilde{\iota}\left(\boldsymbol{\theta}_{\mathbf{d}-1}\right)\right) e
$$

or to

$$
\mathcal{L}\left(W_{e_{1}^{\prime}}, W_{e_{1}}, 1 / 2\right)=c \mathcal{L}\left(W_{e^{\prime}}, W_{e}, 1 / 2\right) .
$$

A tedious computation shows that there exists two matrices $a, b \in G L(\mathbf{d}-$ $1, F)$ such that, for every $h \in G L(\mathbf{d}-1, F)$,

$$
W_{e_{1}^{\prime}}(h)=\check{W}_{e^{\prime}}\left(z^{\prime} a h b\right), W_{e_{1}}(\iota(h))=\check{W}_{e}(z \iota(a h b)),
$$

where $z^{\prime}$ and $z$ are explicit central matrices of ranks $\mathbf{d}-1$ and $\mathbf{d}$. The formulas above are equivalent to

$$
W_{e_{1}^{\prime}}(h)=\omega_{\boldsymbol{\sigma}}\left(z^{\prime}\right) \check{W}_{e^{\prime}}(a h b), W_{e_{1}}(\iota(h))=\omega_{\boldsymbol{\pi}}(z) \check{W}_{e}(\iota(a h b)) .
$$

By the change of variables $h \mapsto a^{-1} h b^{-1}$, we obtain that

$$
\mathcal{L}\left(W_{e_{1}^{\prime}}, W_{e_{1}}, 1 / 2\right)=\overline{\omega_{\boldsymbol{\sigma}}\left(z^{\prime}\right)} \omega_{\boldsymbol{\pi}}(z) \mathcal{L}\left(\check{W}_{e^{\prime}}, \check{W}_{e}, 1 / 2\right) .
$$

Then the relations (6) and (7) imply

$$
c=\overline{\omega_{\boldsymbol{\sigma}}\left(z^{\prime}\right)} \omega_{\boldsymbol{\pi}}(z) \omega_{\boldsymbol{\sigma}}(-1)^{\mathbf{d}-1} \epsilon\left(\boldsymbol{\pi} \times \boldsymbol{\sigma}, 1 / 2, \psi_{F}\right) .
$$

That is the expected relation between $c$ and the $\epsilon$-factor. 


\section{Endoscopy and twisted endoscopy}

4.1. Remark about notations. In the Gross-Prasad conjecture, there is two special orthogonal groups of two spaces of distinct parities. Sometimes, it is better to use a notation that makes clear what are the bigger group and the bigger space and what are the smaller group and the smaller space. In this case, we use the notation of Section 2: $G$ is the bigger group, $V$ is the bigger space, $H$ is the smaller group and $W$ is the smaller space. But sometimes, it is better to use a notation that makes clear what is the even-dimensional space and what is the odd-dimensional space. In this case, we use the following notation: $V$ is the even-dimensional space, $d$ is its dimension and $G$ is its special orthogonal group; $V^{\prime}$ is the odd-dimensional space, $d^{\prime}$ is its dimension and $G^{\prime}$ is its special orthogonal group. In the present section, we use this notation.

4.2. Endoscopy in odd dimensional case. Consider a vector space $V^{\prime}$ over $F$, of finite odd dimensional dimension $d^{\prime}$, with a non-degenerate quadratic form $q^{\prime}$, and denote by $G^{\prime}$ its special orthogonal group. Let $g \in$ $G^{\prime}(F)$ be a strongly regular element (that means its centralizer is a maximal subtorus of $G^{\prime}$ ). The eigenvalues in $\bar{F}$ of $g$ (acting on $V^{\prime} \otimes_{F} \bar{F}$ ) are all distinct. The set $\Lambda(g)$ of those eigenvalues contains 1 and is invariant by $\lambda \mapsto \lambda^{-1}$. We can write

$$
\Lambda(g)=\left\{1, \lambda_{1}^{ \pm 1}, \ldots, \lambda_{\left(d^{\prime}-1\right) / 2}^{ \pm 1}\right\}
$$

Define the stable conjugacy class $C^{s t}(g)$ of $g$ as the set of $g^{\prime} \in G^{\prime}(F)$ for which there exists $x \in G^{\prime}(\bar{F})$ such that $g^{\prime}=x^{-1} g x$. Then, for $g^{\prime} \in G^{\prime}(F), g^{\prime}$ belongs to $C^{s t}(g)$ if and only if $g^{\prime}$ is strongly regular and $\Lambda\left(g^{\prime}\right)=\Lambda(g)$. It is easy to prove that the set of ordinary conjugacy classes by $G^{\prime}(F)$ contained in $C^{s t}(g)$ is finite.

Consider now two others vector spaces $V_{1}^{\prime}$ and $V_{2}^{\prime}$ over $F$, of finite odd dimension $d_{1}^{\prime}$ and $d_{2}^{\prime}$, with non-degenerate quadratic forms $q_{1}^{\prime}$ and $q_{2}^{\prime}$, and denote by $G_{1}^{\prime}$ and $G_{2}^{\prime}$ their special orthogonal groups. We assume

(1) $d_{1}^{\prime}+d_{2}^{\prime}=d^{\prime}+1$;

(2) $G_{1}^{\prime}$ and $G_{2}^{\prime}$ are split over $F$.

Let $g \in G^{\prime}(F), g_{1} \in G_{1}^{\prime}(F)$ and $g_{2} \in G_{2}^{\prime}(F)$ three strongly regular elements. We say that $g$ and $\left(g_{1}, g_{2}\right)$ correspond each other, or that $C^{s t}(g)$ and $C^{s t}\left(g_{1}\right) \times C^{s t}\left(g_{2}\right)$ correspond each other, if and only if $\Lambda(g)=\Lambda\left(g_{1}\right) \cup \Lambda\left(g_{2}\right)$ (then the intersection of the two last sets is reduced to $\{1\})$. It is clear that, given $g_{1}$ and $g_{2}$, there is at most one stable conjugacy class $C^{s t}(g)$ that corresponds to $C^{s t}\left(g_{1}\right) \times C^{s t}\left(g_{2}\right)$. In the opposite direction, given $g$, there is at most a finite set of stable conjugacy classes $C^{s t}\left(g_{1}\right) \times C^{s t}\left(g_{2}\right)$ that correspond to $C^{s t}(g)$.

Let $g, g_{1}$ and $g_{2}$ be as above, assume that $g$ corresponds to $\left(g_{1}, g_{2}\right)$. Then Langlands and Shelstad have defined a transfer factor $\Delta_{G_{1}^{\prime} \times G_{2}^{\prime}, G^{\prime}}\left(g_{1}, g_{2} ; g\right) \in$ $\mathbb{C}^{\times}$. The general definition gives such factor only up to homothety, but in our particular case, there is a natural normalization which gives a well defined 
transfer factor. It is a sign $\Delta_{G_{1}^{\prime} \times G_{2}^{\prime}, G^{\prime}}\left(g_{1}, g_{2} ; g\right) \in\{ \pm 1\}$. As function of $\left(g_{1}, g_{2}\right)$, it depends only of the stable conjugacy classes $C^{s t}\left(g_{1}\right) \times C^{s t}\left(g_{2}\right)$. It is more subtle as function of $g$. It depends only of the ordinary conjugacy class by $G^{\prime}(F)$ of $g$. The set of ordinary conjugacy classes contained in $C^{s t}(g)$ has a natural structure of principal homogeneous space over some cohomology group, which is isomorphic to a finite product of copies of $\mathbb{Z} / 2 \mathbb{Z}$. The datum $\left(g_{1}, g_{2}\right)$ determines some character of this group and the function $g \mapsto \Delta_{G_{1}^{\prime} \times G_{2}^{\prime}, G^{\prime}}\left(g_{1}, g_{2} ; g\right)$ transforms according to this character.

Now consider a function $\theta$ on $G^{\prime}(F)$ which is a finite linear combination of characters of irreducible smooth representations of $G^{\prime}(F)$. Consider similar functions $\theta_{1}$ on $G_{1}^{\prime}(F)$ and $\theta_{2}$ on $G_{2}^{\prime}(F)$. Such functions are invariant by ordinary conjugacy. Assume that $\theta_{1}$ and $\theta_{2}$ are stable, that means they are constant on every stable conjugacy class. We say that $\theta$ is a transfert of $\theta_{1} \times \theta_{2}$ if and only if, for every strongly regular $g \in G^{\prime}(F)$, we have the equality

$$
\begin{aligned}
D^{G^{\prime}}(g)^{1 / 2} \theta(g)= & \sum_{\left(g_{1}, g_{2}\right)} D^{G_{1}^{\prime}}\left(g_{1}\right)^{1 / 2} D^{G_{2}^{\prime}}\left(g_{2}\right)^{1 / 2} \\
& \times \Delta_{G_{1}^{\prime} \times G_{2}^{\prime}, G^{\prime}}\left(g_{1}, g_{2} ; g\right) \theta_{1}\left(g_{1}\right) \theta_{2}\left(g_{2}\right),
\end{aligned}
$$

where the sum is over the pairs $\left(g_{1}, g_{2}\right)$ up to stable conjugacy such that $C^{s t}(g)$ and $C^{s t}\left(g_{1}\right) \times C^{s t}\left(g_{2}\right)$ correspond each other, and the functions $D^{G^{\prime}}$, $D^{G_{1}^{\prime}}$ and $D^{G_{2}^{\prime}}$ are defined as in 2.4. For instance

$$
\left.D^{G^{\prime}}(g)=\mid \operatorname{det}((\operatorname{ad}(g)-1))_{\mid \mathfrak{g}^{\prime}(F) / \mathfrak{g}_{g}^{\prime}(F)}\right)\left.\right|_{F} .
$$

REMARK. We can reverse the order of $G_{1}^{\prime}$ and $G_{2}^{\prime}$. If $g$ corresponds to $\left(g_{1}, g_{2}\right) \in G_{1}^{\prime}(F) \times G_{2}^{\prime}(F)$, it is clear that $g$ corresponds also to $\left(g_{2}, g_{1}\right) \in$ $G_{2}^{\prime}(F) \times G_{1}^{\prime}(F)$. But $\Delta_{G_{1}^{\prime} \times G_{2}^{\prime}, G^{\prime}}\left(g_{1}, g_{2} ; g\right)$ is not always equal to $\Delta_{G_{2}^{\prime} \times G_{1}^{\prime}, G^{\prime}}\left(g_{2}, g_{1} ; g\right)$. In fact, we have

$$
\Delta_{G_{2}^{\prime} \times G_{1}^{\prime}, G^{\prime}}\left(g_{2}, g_{1} ; g\right)=\mu\left(G^{\prime}\right) \Delta_{G_{1}^{\prime} \times G_{2}^{\prime}, G^{\prime}}\left(g_{1}, g_{2} ; g\right),
$$

where

$$
\mu\left(G^{\prime}\right)= \begin{cases}1, & \text { if } G^{\prime} \text { is split; } \\ -1, & \text { if } G^{\prime} \text { is not split. }\end{cases}
$$

In certain sense, we can consider that $G_{1}^{\prime} \times G_{2}^{\prime}$ and $G_{2}^{\prime} \times G_{1}^{\prime}$ are equivalent, but for us, it is better to distinguish the two pairs.

4.3. Twisted endoscopy in the odd dimensional case. Let $V^{\prime}, q^{\prime}$ and $G^{\prime}$ be as in the preceding section. Here, we assume that $G^{\prime}$ is split. There are two useful cases of twisted endoscopy. Let's begin with the most usual. Let $\mathbf{V}$ be a vector space over $F$ of even dimension $\mathbf{d}=d^{\prime}-1$. As in 3.1, we define the group $\mathbf{G}=G L(\mathbf{V})$ and the twisted space $\tilde{\mathbf{G}}$ of nondegenerate bilinear forms on $\mathbf{V}$. For $\gamma \in \tilde{\mathbf{G}}(F)$, let $x_{\gamma}: \mathbf{V} \rightarrow \mathbf{V}^{*}$ and $x_{\gamma}^{*}: \mathbf{V} \rightarrow \mathbf{V}^{*}$ be the two dual isomorphisms such that

$$
\gamma\left(v, v^{\prime}\right)=<v, x_{\gamma}\left(v^{\prime}\right)>=<x_{\gamma}^{*}(v), v^{\prime}>
$$


for every $v, v^{\prime} \in \mathbf{V}$, where $\left\langle., .>\right.$ is the natural pairing between $\mathbf{V}$ and $\mathbf{V}^{*}$. Define an automorphism $y_{\gamma}$ of $\mathbf{V}$ by $y_{\gamma}=\left(x_{\gamma}^{*}\right)^{-1} x_{\gamma}$. We say that $\gamma$ is strongly regular if and only if the centralizer of $\mathbf{G}_{\gamma}$ in $\mathbf{G}$ is a maximal subtorus of G. It is equivalent to say that $y_{\gamma}$ is strongly regular (its centralizer in $\mathbf{G}$ is a maximal subtorus). In this case, let $\Lambda(\gamma)$ be the set of eigenvalues in $\bar{F}$ of $y_{\gamma}$. It is invariant by $\lambda \mapsto \lambda^{-1}$. Define the stable conjugacy class of $\gamma$ as the set of $\gamma^{\prime} \in \tilde{\mathbf{G}}(F)$ for which there exists $x \in \mathbf{G}(\bar{F})$ such that $\gamma^{\prime}=x^{-1} \gamma x$. An element $\gamma^{\prime} \in \tilde{\mathbf{G}}(F)$ belongs to $C^{s t}(\gamma)$ if and only if $\gamma^{\prime}$ is strongly regular and $\Lambda\left(\gamma^{\prime}\right)=\Lambda(\gamma)$

Let $\gamma \in \tilde{\mathbf{G}}(F)$ and $g \in G^{\prime}(F)$ two strongly regular elements. We say that $\gamma$ and $g$ correspond each other, or that $C^{s t}(\gamma)$ and $C^{s t}(g)$ correspond each other if and only if they satisfy the equality

$$
\Lambda(\gamma)=\{-\lambda ; \lambda \in \Lambda(g), \lambda \neq 1\} .
$$

The change of signs is related to the fact that the basic element $\boldsymbol{\theta}_{\mathbf{d}}$ is symplectic. This correspondence is a bijection between stable conjugacy classes of strongly regular elements in $\tilde{\mathbf{G}}(F)$ and in $G^{\prime}(F)$.

Let $\gamma$ and $g$ as before, assume they correspond each other. Then Kottwitz and Shelstad have defined a transfer factor which is a $\operatorname{sign} \Delta_{G^{\prime}, \tilde{\mathbf{G}}}(g, \gamma) \in$ $\{ \pm 1\}$. In fact, in our situation, it is always 1 . Consider a function $\theta$ on $G^{\prime}(F)$ which is a finite linear combination of characters of smooth irreducible representations of $G^{\prime}(F)$ (we apologize for the double meaning of the symbol $\theta$ ). Assume $\theta$ is stable. As in 3.1, we can identify $\tilde{\mathbf{G}}$ with a component of the non-connected group $G L(\mathbf{d})^{+}$. Consider a function $\tilde{\boldsymbol{\theta}}$ on $\tilde{\mathbf{G}}(F)$ which is the restriction to $\tilde{\mathbf{G}}(F)$ of a finite linear combination of characters of smooth irreducible representations of $G L(\mathbf{d})^{+}(F)$. We say that $\tilde{\boldsymbol{\theta}}$ is a transfer of $\theta$ if and only if for every strongly regular $\gamma \in \tilde{\mathbf{G}}(F)$, we have the equality

$$
|2|_{F}^{a} D^{\tilde{\mathbf{G}}}(\gamma)^{1 / 2} \tilde{\boldsymbol{\theta}}(\gamma)=\sum_{g} D^{G^{\prime}}(g)^{1 / 2} \Delta_{G^{\prime}, \tilde{\mathbf{G}}}(g ; \gamma) \theta(g),
$$

where the sum is over the strongly regular elements $g \in G^{\prime}(F)$, up to stable conjugacy, that correspond to $\gamma$, where the functions $D^{\tilde{\mathbf{G}}}$ and $D^{G^{\prime}}$ are as in 3.3 and 2.4 and where $a=-(d-1) / 4$. Because the correspondence between stable conjugacy classes is a bijection, the sum in $g$ is in fact reduced to a unique term.

REMARK. Let $\mathbf{T}$ be the centralizer of $\mathbf{G}_{\gamma}$ in $\mathbf{G}$. Then we have

$$
|2|_{F}^{a}=\left|\operatorname{det}\left(\left(1-\theta_{\gamma}\right)_{\mid \boldsymbol{t} / \mathbf{g}_{\gamma}}\right)\right|_{F}^{-1} .
$$

Now consider the second useful case of twisted endoscopy. Let $\mathbf{V}^{\prime}$ be a vector space over $F$ of odd dimension $\mathbf{d}^{\prime}=d^{\prime}$. We introduce similarly the group $\mathbf{G}^{\prime}=G L\left(\mathbf{V}^{\prime}\right)$ and the twisted space $\tilde{\mathbf{G}}^{\prime}$. For $\gamma \in \tilde{\mathbf{G}}^{\prime}(F)$ we define as before the set $\Lambda(\gamma)$ and, if $\gamma$ is strongly regular, the stable conjugacy class $C^{s t}(\gamma)$. In this case, the set $\Lambda(\gamma)$ contains always 1 . Let $\gamma \in \tilde{\mathbf{G}}^{\prime}(F)$ and 
$g \in G^{\prime}(F)$ two strongly regular elements. We say that $\gamma$ and $g$ correspond each other, or that $C^{s t}(\gamma)$ and $C^{s t}(g)$ correspond each other if and only if they satisfy the equality $\Lambda(g)=\Lambda(\gamma)$. There is no sign here because $\boldsymbol{\theta}_{\mathbf{d}^{\prime}}$ is now symmetric.

Let $\gamma$ and $g$ as before, assume they correspond each other. Then Kottwitz and Shelstad have defined a transfer factor which is a $\operatorname{sign} \Delta_{G^{\prime}, \tilde{\mathbf{G}}^{\prime}}$ $(g, \gamma) \in\{ \pm 1\}$. It is more complicated as in the preceding case. The factor $\Delta_{G^{\prime}, \tilde{\mathbf{G}}^{\prime}}(g ; \gamma)$ depends only of the stable conjugacy class of $g$ and of the ordinary conjugacy class by $\mathbf{G}^{\prime}(F)$ of $\gamma$. As in the preceding section, the set of ordinary conjugacy classes contained in $C^{s t}(\gamma)$ has a natural structure of principal homogeneous space over some cohomology group, which is isomorphic to a finite product of copies of $\mathbb{Z} / 2 \mathbb{Z}$. The datum $g$ determines some character of this group and the function $\gamma \mapsto \Delta_{G^{\prime}, \tilde{\mathbf{G}}^{\prime}}(g ; \gamma)$ transforms according to this character.

Consider a function $\theta$ on $G^{\prime}(F)$ and a function $\tilde{\boldsymbol{\theta}}$ on $\tilde{\mathbf{G}}^{\prime}(F)$ satisfying similar properties as above. We say that $\tilde{\boldsymbol{\theta}}$ is a transfer of $\theta$ if and only if for every strongly regular $\gamma \in \tilde{\mathbf{G}}(F)$, we have the equality (1), where now $a=-(d+1) / 4$ (the sum in $g$ is again reduced to a unique term).

4.4. Endoscopy in the even dimensional case. Consider a vector space $V$ over $F$, of finite even dimension $d$, provided with a non-degenerate quadratic form $q$, and let $G$ be its special orthogonal group. Let $g \in G(F)$ be a strongly regular element. The eigenvalues in $\bar{F}$ of $g$ are all distinct. The set $\Lambda(g)$ of those eigenvalues is of the form

$$
\Lambda(g)=\left\{\lambda_{1}^{ \pm 1}, \ldots, \lambda_{d / 2}^{ \pm 1}\right\}
$$

Define the stable conjugacy class $C^{s t}(g)$ of $g$ as in 4.2 . Then, if $g^{\prime}$ belongs to $C^{s t}(g)$, we have $\Lambda\left(g^{\prime}\right)=\Lambda(g)$. But this condition is not sufficient. In fact the set of strongly regular $g^{\prime} \in G(F)$ such that $\Lambda\left(g^{\prime}\right)=\Lambda(g)$ is the union of two stable conjugacy classes, which are conjugate by an element of determinant -1 of the full orthogonal group.

Consider two others vector spaces $V_{1}$ and $V_{2}$ over $F$, of finite even dimension $d_{1}$ and $d_{2}$, with non-degenerate quadratic forms $q_{1}$ and $q_{2}$, and denote by $G_{1}$ and $G_{2}$ their special orthogonal groups. We assume

(1) $d_{1}+d_{2}=d$

(2) $G_{1}$ and $G_{2}$ are quasi-split over $F$;

(3) $\delta=\delta_{1} \delta_{2}$, where $\delta, \delta_{1}, \delta_{2} \in F^{\times} / F^{\times, 2}$ are the discriminants of $q, q_{1}$ and $q_{2}$ (by convention, $\delta_{i}=1$ if $d_{i}=0$ for $i=1$ or 2 ).

Let $g \in G(F), g_{1} \in G_{1}(F)$ and $g_{2} \in G_{2}(F)$ three strongly regular elements. In first approximation, we can say that $g$ and $\left(g_{1}, g_{2}\right)$ correspond each other, or that $C^{s t}(g)$ and $C^{s t}\left(g_{1}\right) \times C^{s t}\left(g_{2}\right)$ correspond each other, if and only if $\Lambda(g)=\Lambda\left(g_{1}\right) \cup \Lambda\left(g_{2}\right)$. But this correspondence is not sufficiently fine, because the sets of eigenvalues does not determine the stable conjugacy classes. We must define a finer correspondence. I will give very briefly the definition. 
We fix once for all maximal subtori $T$ of $G, T_{1}$ of $G_{1}, T_{2}$ of $G_{2}$, defined over $\bar{F}$. Consider the group $X^{*}(T)$ of algebraic characters of $T$, defined over $\bar{F}$. We can fix a basis $\mathcal{B}$ of $X^{*}(T)$ over $\mathbb{Z}$ such that, for every $t \in T(\bar{F})$, the set of eigenvalues of the action of $t$ in $V \otimes_{F} \bar{F}$ is

$$
\left\{\left(x^{*}(t)\right)^{ \pm 1} ; x^{*} \in \mathcal{B}\right\} .
$$

We fix similarly basis $\mathcal{B}_{1}$ of $X^{*}\left(T_{1}\right)$ and $\mathcal{B}_{2}$ of $X^{*}\left(T_{2}\right)$. Next, fix a bijection between $\mathcal{B}$ and $\mathcal{B}_{1} \sqcup \mathcal{B}_{2}$. By linearity, it defined an isomorphism $X^{*}(T) \rightarrow$ $X^{*}\left(T_{1}\right) \oplus X^{*}\left(T_{2}\right)$, then an isomorphism $\iota: T_{1} \times T_{2} \rightarrow T$, defined over $\bar{F}$. Denote by $W$ the Weyl group of $G$ relative to $T$. Our element $g$ is conjugate in $G(\bar{F})$ to an element $t \in T(\bar{F})$. Similarly, $g_{1}$ and $g_{2}$ are conjugate to $t_{1} \in T_{1}(\bar{F})$ and $t_{2} \in T_{2}(\bar{F})$. Then we say that $g$ and $\left(g_{1}, g_{2}\right)$ correspond each other if and only if the images of $t$ and of $\iota\left(t_{1}, t_{2}\right)$ in $T / W$ are equal.

This correspondence has similar properties as in the odd dimensional case.

As we said in 4.2, the general definition of transfer factor gives only a function up to homothety. To normalize it, some choice are needed. I don't explain that, but it appears that the choice of an element $\nu \in F^{\times} / F^{\times, 2}$ is sufficient to normalize the transfer factor. Then, we choose such $\nu$ and we have a transfer factor $\Delta_{G_{1} \times G_{2}, G}\left(g_{1}, g_{2} ; g\right) \in\{ \pm 1\}, g, g_{1}$ and $g_{2}$ being as above, assuming that $g$ corresponds to $\left(g_{1}, g_{2}\right)$.

It is convenient to define a sign $\mu(G)$ similar to the sign defined in 4.2. The definition depends now on $\nu$. Consider the line $Z(\nu)=F$ with the quadratic form $q_{Z(\nu)}\left(x, x_{1}\right)=-2 \nu x x_{1}$. Denote by $G^{\prime}(\nu)$ the special orthogonal group of the orthogonal sum $V \oplus Z(\nu)$. We define

$$
\mu(G)= \begin{cases}1, & \text { if } G^{\prime}(\nu) \text { is split over } F \\ -1, & \text { if } G^{\prime}(\nu) \text { is not split over } F .\end{cases}
$$

Recall that the elementary theory of quadratic forms over local fields says that $V$ is always the orthogonal direct sum of hyperbolic planes and of an anisotropic space of dimension $d_{a n}=0,2$ or 4 . When $d_{a n}=0$, then $G$ is split and $G^{\prime}(\nu)$ is also split whatever $\nu$. When $d_{a n}=4$, then $G$ is not quasisplit and $G^{\prime}(\nu)$ is not split whatever $\nu$. But if $d_{a n}=2$, then $G$ is quasi-split and $G^{\prime}(\nu)$ can be split or not split according to $\nu$.

Now consider a function $\theta$ on $G(F)$ which is a finite linear combination of characters of irreducible smooth representations of $G(F)$. Consider similar functions $\theta_{1}$ on $G_{1}(F)$ and $\theta_{2}$ on $G_{2}(F)$. Assume that $\theta_{1}$ and $\theta_{2}$ are stable. We say that $\theta$ is a transfert of $\theta_{1} \times \theta_{2}$ if and only if, for every strongly regular $g \in G(F)$, we have an equality similar to $4.2(3)$.

REMARK. As in the odd-dimensional case, we can reverse the order of $G_{1}$ and $G_{2}$. An equality similar to 4.2 (4) remains true.

4.5. Twisted endoscopy in the even-dimensional case. Let $V, q$ and $G$ be as in the preceding section. Here, we assume that $G$ is quasi-split. Let $\mathbf{V}$ be a vector space over $F$ of dimension $\mathbf{d}=d$. We introduce the 
group $\mathbf{G}=G L(\mathbf{V})$ and the twisted space $\tilde{\mathbf{G}}$. For a strongly regular element $\gamma \in \tilde{\mathbf{G}}(F)$, we define the set $\Lambda(\gamma)$ as in 4.3 .

Let $\gamma \in \tilde{\mathbf{G}}(F)$ and $g \in G(F)$ two strongly regular elements. We say that $\gamma$ and $g$ corresponds each other, or that $C^{s t}(\gamma)$ and $C^{s t}(g)$ corresponds each other if and only if they satisfy the equality

$$
\Lambda(\gamma)=\{-\lambda ; \lambda \in \Lambda(g)\}
$$

In fact, for each $C^{s t}(g)$, there is a unique corresponding $C^{s t}(\gamma)$. The application $C^{s t}(g) \mapsto C^{s t}(\gamma)$ is not surjective. On its image, the fibers have two elements (the two stable conjugacy classes determined by the set of eigenvalues).

Let $\gamma$ and $g$ as before, assume they correspond each other. Then Kottwitz and Shelstad have defined a transfer factor $\Delta_{G, \tilde{\mathbf{G}}}(g, \gamma) \in\{ \pm 1\}$ (that is not always 1). Consider a function $\theta$ on $G(F)$ which is a finite linear combination of characters of smooth irreducible representations of $G(F)$. Assume $\theta$ is stable. Consider a function $\tilde{\boldsymbol{\theta}}$ on $\tilde{\mathbf{G}}(F)$ which is, as in 4.3 , the restriction to $\tilde{\mathbf{G}}(F)$ of a finite linear combination of characters of smooth irreducible representations of $G L(\mathbf{d})^{+}(F)$. We say that $\tilde{\boldsymbol{\theta}}$ is a transfer of $\theta$ if and only if for every strongly regular $\gamma \in \tilde{\mathbf{G}}(F)$, an equality similar to 4.3 (1) is satisfied, where $a=-d / 4$.

4.6. Parametrization and endoscopy: the odd-dimensional case. We can now state more a precise version of the conjectures of 1.3 . Let $V^{\prime}, q^{\prime}$, $G^{\prime}$ be as in 4.2 ( $d^{\prime}$ is odd). We use the definitions and notations of 1.3. Let $\varphi^{\prime} \in \Phi_{\text {temp }}^{\text {orth }}\left(d^{\prime}\right)$. The central elements \pm 1 of $S p\left(d^{\prime}-1, \mathbb{C}\right)$ are contained in $S\left(\varphi^{\prime}\right)$. Denote by $z_{\varphi^{\prime}}$ the image of -1 in $S\left(\varphi^{\prime}\right) / S\left(\varphi^{\prime}\right)^{0}$. For $\mu \in\{ \pm 1\}$, denote by $\mathcal{E}^{\mu}\left(\varphi^{\prime}\right)$ the set of characters $\epsilon^{\prime}$ of $S\left(\varphi^{\prime}\right) / S\left(\varphi^{\prime}\right)^{0}$ such that $\epsilon^{\prime}\left(z_{\varphi^{\prime}}\right)=\mu$. We have stated that to $\varphi^{\prime}$ is associated a packet $\Pi^{G^{\prime}}\left(\varphi^{\prime}\right)$ of irreducible tempered representations of $G^{\prime}(F)$. We add now that there is a bijection

$$
\begin{aligned}
\mathcal{E}^{\mu\left(G^{\prime}\right)}\left(\varphi^{\prime}\right) & \rightarrow \Pi^{G^{\prime}}\left(\varphi^{\prime}\right) \\
\epsilon^{\prime} & \mapsto \pi\left(\varphi^{\prime}, \epsilon^{\prime}\right) .
\end{aligned}
$$

Let's remark that $\mathcal{E}^{-}\left(\varphi^{\prime}\right)$ can be empty (when the center of $S p\left(d^{\prime}-1, \mathbb{C}\right.$ ) in included in $\left.S\left(\varphi^{\prime}\right)^{0}\right)$. In this case, $\Pi^{G^{\prime}}\left(\varphi^{\prime}\right)$ is empty if $\mu\left(G^{\prime}\right)=-1$.

For $s^{\prime} \in S\left(\varphi^{\prime}\right) / S\left(\varphi^{\prime}\right)^{0}$, we define the following linear combination of characters of irreducible representations

$$
\theta^{G^{\prime}}\left(\varphi^{\prime}, s^{\prime}\right)=\sum_{\epsilon^{\prime} \in \mathcal{E}^{\mu\left(G^{\prime}\right)}\left(\varphi^{\prime}\right)} \epsilon^{\prime}\left(s^{\prime}\right) \theta_{\pi\left(\varphi^{\prime}, \epsilon^{\prime}\right)} .
$$

Assume $\mu\left(G^{\prime}\right)=1$. Then $G^{\prime}$ is split. In this case, a first required property is

(3) the functions $\theta^{G^{\prime}}\left(\varphi^{\prime}, 1_{\varphi^{\prime}}\right)$ is stable, where $1_{\varphi^{\prime}}$ is the identity element of $S\left(\varphi^{\prime}\right) / S\left(\varphi^{\prime}\right)^{0}$. 
Denote by $\varphi^{\prime}: W_{D F} \rightarrow G L\left(d^{\prime}-1, \mathbb{C}\right)$ the composition of $\varphi^{\prime}$ and of the natural embedding $S p\left(d^{\prime}-1, \mathbb{C}\right) \rightarrow G L\left(d^{\prime}-1, \mathbb{C}\right)$. The local conjecture of Langlands for the group $G L\left(d^{\prime}-1\right)$ has been proved by Harris-Taylor and Henniart ([HT], [H] ). It associates to $\varphi^{\prime}$ an irreducible representation $\boldsymbol{\pi}\left(\boldsymbol{\varphi}^{\prime}\right)$ of $G L\left(d^{\prime}-1, F\right)$. This representation is tempered and autodual, so we can extend it as in 3.3 in a representation $\boldsymbol{\pi}\left(\varphi^{\prime}\right)^{+}$of $G L\left(d^{\prime}-1\right)^{+}(F)$. We denote by $\tilde{\boldsymbol{\pi}}\left(\varphi^{\prime}\right)$ its restriction to $G L\left(d^{\prime}-1, F\right) \theta_{d-1}$. Introducing a space $\mathbf{V}$ of dimension $\mathbf{d}=d^{\prime}-1$ as in 4.3 , we identify $\theta_{\tilde{\boldsymbol{\pi}}\left(\boldsymbol{\varphi}^{\prime}\right)}$ to a function on $\tilde{\mathbf{G}}(F)$. We require

(4) there is some scalar $c\left(\varphi^{\prime}\right) \in \mathbb{C}$ such that $\left|c\left(\varphi^{\prime}\right)\right|=1$ and that $c\left(\varphi^{\prime}\right) \theta_{\tilde{\boldsymbol{\pi}}\left(\varphi^{\prime}\right)}$ is the transfer of $\theta^{G^{\prime}}\left(\varphi^{\prime}, 1_{\varphi^{\prime}}\right)$.

Denote by $\varphi_{>}^{\prime}: W_{D F} \rightarrow G L\left(d^{\prime}, \mathbb{C}\right)$ the direct sum of $\varphi^{\prime}$ and of the trivial one-dimensional representation of $W_{D F}$. We define similarly the function $\theta_{\tilde{\boldsymbol{\pi}}\left(\boldsymbol{\varphi}_{>}^{\prime}\right)}$ on $\tilde{\mathbf{G}}^{\prime}(F)$, where $\tilde{\mathbf{G}}^{\prime}$ is as in $4.3\left(\right.$ then $\left.\mathbf{G}^{\prime} \simeq G L\left(d^{\prime}\right)\right)$. We require

(5) there is some scalar $c_{>}\left(\varphi^{\prime}\right) \in \mathbb{C}$ such that $\left|c_{>}\left(\varphi^{\prime}\right)\right|=1$ and that $c_{>}\left(\varphi^{\prime}\right) \theta_{\tilde{\boldsymbol{\pi}}\left(\varphi_{>}^{\prime}\right)}$ is the transfer of $\theta^{G^{\prime}}\left(\varphi^{\prime}, 1_{\varphi^{\prime}}\right)$.

We have stated the assertion (4) because it is the most usual form of twisted endoscopy for odd-dimensional special orthogonal groups. But for our problem, we use only the assertion (5).

Now we remove the assumption $\mu\left(G^{\prime}\right)=1$. Consider two groups $G_{1}^{\prime}$ and $G_{2}^{\prime}$ as in 4.2. Let $\varphi_{1}^{\prime} \in \Phi_{\text {temp }}^{\text {orth }}\left(d_{1}^{\prime}\right)$ and $\varphi_{2}^{\prime} \in \Phi_{\text {temp }}^{\text {orth }}\left(d_{2}^{\prime}\right)$. There is a natural embedding

$$
S p\left(d_{1}^{\prime}-1, \mathbb{C}\right) \times S p\left(d_{2}^{\prime}-1, \mathbb{C}\right) \rightarrow S p\left(d^{\prime}-1, \mathbb{C}\right)
$$

well defined up to conjugacy. Assume that $\varphi^{\prime}$ is equal to the composition of $\left(\varphi_{1}^{\prime}, \varphi_{2}^{\prime}\right)$ with this embedding. Consider the image in $S p\left(d^{\prime}-1, \mathbb{C}\right)$ of the product of the central element $1 \in S p\left(d_{1}^{\prime}-1, \mathbb{C}\right)$ and of the central element $-1 \in S p\left(d_{2}^{\prime}-1, \mathbb{C}\right)$. It belongs to $S\left(\varphi^{\prime}\right)$. Denote by $s^{\prime}$ its image in $S\left(\varphi^{\prime}\right) / S\left(\varphi^{\prime}\right)^{0}$. Then we require

(6) for every $\mu \in\{ \pm 1\}$, there is some scalar $\gamma^{\mu}\left(\varphi_{1}^{\prime}, \varphi_{2}^{\prime}\right) \in \mathbb{C}$ such that $\left|\gamma^{\mu}\left(\varphi_{1}^{\prime}, \varphi_{2}^{\prime}\right)\right|=1$ and that $\gamma^{\mu\left(G^{\prime}\right)}\left(\varphi_{1}^{\prime}, \varphi_{2}^{\prime}\right) \theta^{G^{\prime}}\left(\varphi^{\prime}, s^{\prime}\right)$ is the transfer of $\theta^{G_{1}^{\prime}}\left(\varphi_{1}^{\prime}, 1_{\varphi_{1}^{\prime}}\right) \times \theta^{G_{2}^{\prime}}\left(\varphi_{2}^{\prime}, 1_{\varphi_{2}^{\prime}}\right)$.

Let's remark that the scalars $c\left(\varphi^{\prime}\right)$ and $c_{>}\left(\varphi^{\prime}\right)$ of (4) and (5) are well determined. But the assertion (6) is less precise: we can change the scalar $\gamma^{\mu}\left(\varphi_{1}^{\prime}, \varphi_{2}^{\prime}\right)$ if we change the bijections (1). In the opposite direction, if we fix the scalars $\gamma^{\mu}\left(\varphi^{\prime}, \varphi^{\prime \prime}\right)$ for all possible $\mu, G_{1}^{\prime}, G_{2}^{\prime}, \varphi_{1}^{\prime}, \varphi_{2}^{\prime}$, the bijections (1) is well determined. In fact, assuming the preceding assertions, the following proposition fix the scalars.

Proposition. The scalars $c_{>}\left(\varphi^{\prime}\right)$ of (5) are equal to 1 . Up to change of the bijections (1), we can assume that the scalars $\gamma^{\mu}\left(\varphi_{1}^{\prime}, \varphi_{2}^{\prime}\right)$ of (6) are equal to $\mu$. 
It is convenient to state here this proposition, but we prove it at the same time as the main theorem 5.4 below.

4.7. Parametrization and endoscopy: the even-dimensional case. Let $V, q, G$ be as in 4.4 . The dimension $d$ is even. We must consider the complex group $S O(d, \mathbb{C})$ as the Langlands dual of $G$. This statement contains some implicit data. In practice, those data are as follows. We fix a maximal subtorus $T$ of $G$ defined over $\bar{F}$ and a basis $\mathcal{B}$ of $X^{*}(T)$ satisfying the same properties as in 4.4 . The group $S O(d, \mathbb{C})$ is a subgroup of $G L(d, \mathbb{C})$ and acts on $\mathbb{C}^{d}$. Fix a maximal subtorus $\hat{T}$ of $S O(d, \mathbb{C})$ and a basis $\hat{\mathcal{B}}$ of $X^{*}(\hat{T})$ such that, for every $\hat{t} \in \hat{T}$, the set of eigenvalues of the action of $\hat{t}$ in $\mathbb{C}^{d}$ is

$$
\left\{\left(x^{*}(\hat{t})\right)^{ \pm 1} ; x^{*} \in \hat{\mathcal{B}}\right\} .
$$

The essential data is a perfect duality between $X^{*}(T)$ and $X^{*}(\hat{T})$ for which $\mathcal{B}$ and $\hat{\mathcal{B}}$ are dual basis.

Recall that $\delta \in F^{\times} / F^{\times, 2}$ is the discriminant of $q$. Let $\varphi \in \Phi_{\text {temp }}^{\text {orth }}(d, \delta)$. The central elements \pm 1 of $S O(d, \mathbb{C})$ are contained in $S(\varphi)$. Denote by $z_{\varphi}$ the image of -1 in $S(\varphi) / S(\varphi)^{0}$. For $\mu \in\{ \pm 1\}$, denote by $\mathcal{E}^{\mu}(\varphi)$ the set of characters $\epsilon$ of $S(\varphi) / S(\varphi)^{0}$ such that $\epsilon\left(z_{\varphi}\right)=\mu$. To $\varphi$ is associated a packet $\Pi^{G}(\varphi)$ of irreducible tempered representations of $G(F)$. We require that there exists a bijection

$$
\begin{aligned}
\mathcal{E}^{\mu(G)}(\varphi) & \rightarrow \Pi^{G}(\varphi) \\
\epsilon & \mapsto \pi(\varphi, \epsilon) .
\end{aligned}
$$

REMARK. We have said in 4.4 that, if $d_{a n}(V)=0$, resp. $4, \mu(G)=1$, resp. -1 . In this case, the set $\mathcal{E}^{\mu(G)}(\varphi)$ is well defined. But, if $d_{a n}(V)=2$, $\mu(G)$ can be \pm 1 according to the choice of $\nu$. Then, according to this choice, the same set $\Pi^{G}(\varphi)$ can be parametrized by the two distinct sets $\mathcal{E}^{+}(\varphi)$ or $\mathcal{E}^{-}(\varphi)$.

For $s \in S(\varphi) / S(\varphi)^{0}$, we define $\theta^{G}(\varphi, s)$ by an equality similar to 4.6 (2). Assume first $\mu(G)=1$. Then $G$ is quasi-split. As in 4.6 , we require

(2) the functions $\theta^{G}\left(\varphi, 1_{\varphi}\right)$ is stable.

Denote by $\varphi: W_{D F} \rightarrow G L(d, \mathbb{C})$ the composition of $\varphi$ with of the natural embedding $O(d, \mathbb{C}) \rightarrow G L(d, \mathbb{C})$. As in 4.6 , we define a function $\theta_{\tilde{\boldsymbol{\pi}}(\boldsymbol{\varphi})}$ on $\tilde{\mathbf{G}}(F)$, where $\tilde{\mathbf{G}}$ is as in 4.5 . We require

(3) there is some scalar $c(\varphi) \in \mathbb{C}$ such that $|c(\varphi)|=1$ and that $c(\varphi) \theta_{\tilde{\boldsymbol{\pi}}(\varphi)}$ is the transfer of $\theta^{G}\left(\varphi, 1_{\varphi}\right)$.

Now we remove the assumption $\mu(G)=1$. Consider two groups $G_{1}$ and $G_{2}$ as in 4.4. Let $\varphi_{1} \in \Phi_{\text {temp }}^{\text {orth }}\left(d_{1}, \delta_{1}\right)$ and $\varphi_{2} \in \Phi_{\text {temp }}^{\text {orth }}\left(d_{2}, \delta_{2}\right)$. There is a natural embedding

$$
O\left(d_{1}, \mathbb{C}\right) \times O\left(d_{2}, \mathbb{C}\right) \rightarrow O(d, \mathbb{C}),
$$

but well defined only up to conjugacy by the full orthogonal group $O(d, \mathbb{C})$. We need to be more precise. We have fixed above some data: $T, \mathcal{B}, \hat{T}, \hat{\mathcal{B}}$ and 
a perfect duality between $X^{*}(T)$ and $X^{*}(\hat{T})$. Of course, we assume fixed similar data $T_{1}, \mathcal{B}_{1}$ etc. . f for the other groups. In 4.4 , we have fixed data $T, \mathcal{B}, T_{1}$ etc... We assume there are the same as before. Then, using the three perfect dualities, the isomorphism $\iota: T_{1} \times T_{2} \rightarrow T$ of 4.4 defines an isomorphism $\hat{\iota}: \hat{T} \rightarrow \hat{T}_{1} \times \hat{T}_{2}$. We choose the embedding (4) such that its restriction to $\hat{T}_{1} \times \hat{T}_{2}$ is $\hat{\iota}^{-1}$.

Assume that $\varphi$ is equal to the composition of $\left(\varphi_{1}, \varphi_{2}\right)$ and this embedding. Consider the image in $S O(d, \mathbb{C})$ of the product of the central element $1 \in S O\left(d_{1}, \mathbb{C}\right)$ and of the central element $-1 \in S O\left(d_{2}, \mathbb{C}\right)$. It belongs to $S(\varphi)$. Denote by $s$ its image in $S(\varphi) / S(\varphi)^{0}$. Then we require

(5) for every $\mu \in\{ \pm 1\}$, there is some scalar $\gamma^{\mu}\left(\varphi_{1}, \varphi_{2}\right) \in \mathbb{C}$ such that $\left|\gamma^{\mu}\left(\varphi_{1}, \varphi_{2}\right)\right|=1$ and that $\gamma^{\mu(G)}\left(\varphi_{1}, \varphi_{2}\right) \theta^{G}(\varphi, s)$ is the transfer of $\theta^{G_{1}}\left(\varphi_{1}\right.$, $\left.1_{\varphi_{1}}\right) \times \theta^{G_{2}}\left(\varphi_{2}, 1_{\varphi_{2}}\right)$.

As in 4.6 , we can be more precise concerning the scalars. Recall that the center of $G(F)$ is $\{ \pm 1\}$ if $d \neq 2$ and, in any case, contains $\{ \pm 1\}$. Then, for every irreducible smooth representation $\pi$ of $G(F)$, the value $\omega_{\pi}(-1)$ of the central character of $\pi$ at the central element -1 is well defined, and, of course, it is a sign \pm 1 .

Proposition. (i) For every $\varphi \in \Phi_{\text {temp }}^{\text {orth }}(d, \delta)$, there is a sign $\zeta(\varphi)= \pm 1$ such that for every $\pi \in \Pi^{G}(\varphi)$, we have $\omega_{\pi}(-1)=\zeta(\varphi)$ (independently of $\mu(G)$ ).

(ii) The scalar $c(\varphi)$ of (3) is equal to $\zeta(\varphi) \epsilon\left(\boldsymbol{\pi}(\boldsymbol{\varphi}), 1 / 2, \psi_{F}\right)^{-1}$.

(iii) Up to change of the bijections (1), we can assume that the scalars $\gamma^{\mu}\left(\varphi_{1}, \varphi_{2}\right)$ of (5) are all equal to 1.

The first assertion is proved directly. The two others are proved at the same time as the main theorem.

REMARK. (6) As in 3.4, it is reasonable that the scalar $c(\varphi)$ depends on $\psi_{F}$ because the normalization of $\tilde{\boldsymbol{\pi}}(\boldsymbol{\varphi})$ depends on it.

(7) $\epsilon\left(\boldsymbol{\pi}(\boldsymbol{\varphi}), 1 / 2, \psi_{F}\right)$ is always a four root of unity, but not always \pm 1 . So the same holds for the scalar $c(\varphi)$.

4.8. The present state of the conjectures. Arthur has now proved a substantial part of the conjectures stated in the Sections 4.2 to 4.7. The proofs will appear in the forthcoming paper [A]. More precisely, in the odddimensional case, he has proved all conjectures for split groups $G^{\prime}$. There is no doubt that the same methods can be used in the non-split case, and may be the final Arthur's article will include this case. In the even-dimensional case, there is a slight problem: it is difficult to distinguish two representations of $G(F)$ that are not equivalent but are conjugate by an element of the full orthogonal group. Similarly, it is difficult to distinguish two parameters $\varphi$ that are not equivalent modulo conjugacy by $S O(d, \mathbb{C})$, but becomes equivalent modulo conjugacy by $O(d, \mathbb{C})$. It is possible to state weaker conjectures where we identify two representations of $G(F)$ conjugate by an element of the full orthogonal group and, similarly, we identify two 
parameters conjugate by an element of $O(d, \mathbb{C})$. Arthur prove this weaker conjecture when the group $G$ is quasi-split. As in the odd-dimensional case, the same proof holds certainly if $G$ is not quasi-split. I don't know if it is possible to prove the stronger form of the conjectures without substantial additional work.

\section{The precise form of the Gross-Prasad conjecture}

5.1. Description of some centralizers. Let $N \geq 1$ be an integer and let $\boldsymbol{\varphi}: W_{D F} \rightarrow G L(N, \mathbb{C})$ be a continuous semi-simple homomorphism whose restriction to $S L(2, \mathbb{C})$ is algebraic. Assume that either the image of $\boldsymbol{\varphi}$ in contained in $O(N, \mathbb{C})$, or $N$ is even and the image of $\varphi$ is contained in $\operatorname{Sp}(N, \mathbb{C})$. Then $\varphi$ is conjugate to the homomorphism $w \mapsto \varphi^{\theta}(w)=$ ${ }^{t} \boldsymbol{\varphi}(w)^{-1}$. Consider an irreducible component $\boldsymbol{\psi}$ of $\boldsymbol{\varphi}$, which takes its values in some group $G L\left(N_{\boldsymbol{\psi}}, \mathbb{C}\right)$. Then $\boldsymbol{\psi}^{\theta}$ is also such irreducible component. If $\boldsymbol{\psi}^{\theta} \simeq \boldsymbol{\psi}$, its implies that, up to conjugacy, either $\boldsymbol{\psi}$ takes its values in $O\left(N_{\boldsymbol{\psi}}, \mathbb{C}\right)$, or $N_{\boldsymbol{\psi}}$ is even and $\boldsymbol{\psi}$ takes its values in $S p\left(N_{\boldsymbol{\psi}}, \mathbb{C}\right)$ (the two cases are disjoint). Then we can decompose $\varphi$ as

$$
\boldsymbol{\varphi}=\left(\oplus_{i \in I^{\text {orth }}} l_{i} \boldsymbol{\varphi}_{i}\right) \oplus\left(\oplus_{i \in I^{\text {symp }}} l_{i} \boldsymbol{\varphi}_{i}\right) \oplus\left(\oplus_{j \in J} l_{j}\left(\boldsymbol{\varphi}_{j} \oplus \boldsymbol{\varphi}_{j}^{\theta}\right)\right),
$$

where:

- $I^{\text {orth }}, I^{\text {symp }}$ and $J$ are finite disjoint sets;

- for $i \in I^{\text {orth }}, \boldsymbol{\varphi}_{i}$ is an irreducible homomorphism from $W_{D F}$ to some $O\left(N_{i}, \mathbb{C}\right)$ and $l_{i} \geq 1$ is its multiplicity; if $i \neq i^{\prime}, \varphi_{i}$ is not equivalent to $\varphi_{i^{\prime}}$;

- for $i \in I^{\text {symp }}, \varphi_{i}$ is an irreducible homomorphism from $W_{D F}$ to some $\operatorname{Sp}\left(N_{i}, \mathbb{C}\right)$ with $N_{i}$ even, and $l_{i} \geq 1$ is its multiplicity; if $i \neq i^{\prime}$, $\boldsymbol{\varphi}_{i}$ is not equivalent to $\boldsymbol{\varphi}_{i^{\prime}}$;

- for $j \in J, \boldsymbol{\varphi}_{j}$ is an irreducible homomorphism from $W_{D F}$ to some $G L\left(N_{j}, \mathbb{C}\right)$ and $\varphi_{j}^{\theta}$ is not equivalent to $\varphi_{j} ; l_{j} \geq 1$ is its multiplicity; if $j \neq j^{\prime}, \boldsymbol{\varphi}_{j}$ is equivalent neither to $\boldsymbol{\varphi}_{j^{\prime}}$ nor to $\boldsymbol{\varphi}_{j^{\prime}}^{\theta}$.

It is easy to compute the centralizer in $G L(N, \mathbb{C})$ of the image of $\varphi$. It is isomorphic to

$$
\left(\prod_{i \in I^{\text {orth }}} G L\left(l_{i}, \mathbb{C}\right)\right) \times\left(\prod_{i \in I^{\text {Iymp }}} G L\left(l_{i}, \mathbb{C}\right)\right) \times\left(\prod_{j \in J}\left(G L\left(l_{j}, \mathbb{C}\right) \times G L\left(l_{j}, \mathbb{C}\right)\right)\right) .
$$

Consider more precisely the case where $N$ is even and $\varphi$ takes its values in $S p(N, \mathbb{C})$. The centralizer in $S p(N, \mathbb{C})$ of the image of $\boldsymbol{\varphi}$ is the intersection with $S p(N, \mathbb{C})$ of the group above. Let $i \in I^{\text {orth }}$. The group $G L\left(l_{i}, \mathbb{C}\right)$ that appears is in fact the group of automorphisms of the space $U_{i}$ of intertwining homomorphisms from $\varphi_{i}$ to $\varphi$. Because the space of $\varphi_{i}$ is provided with a quadratic form and the one of $\varphi$ is provided with a symplectic form, there is a natural symplectic form on $U_{i}$. This imply first that $l_{i}$ is even. Then the intersection of $G L\left(l_{i}, \mathbb{C}\right)$ with $S p(N, \mathbb{C})$ is the symplectic group of this 
symplectic form, in other words a group $S p\left(l_{i}, \mathbb{C}\right)$. Now let $i \in I^{s y m p}$. For the same reason, the space $U_{i}$ defined as before is provided with a quadratic form, and the intersection of $G L\left(l_{i}, \mathbb{C}\right)$ with $S p(N, \mathbb{C})$ is isomorphic to the orthogonal group of this quadratic form, in other words to a group $O\left(l_{i}, \mathbb{C}\right)$. Lastly, let $j \in J$. Then the group $G L\left(l_{j}, \mathbb{C}\right) \times G L\left(l_{j}, \mathbb{C}\right)$ that appears is the product of the groups of automorphisms of two spaces $U_{j}$ and $U_{j}^{\theta}$, where $U_{j}$, resp. $U_{j}^{\theta}$, is the space of intertwining homomorphisms from $\boldsymbol{\varphi}_{j}$, resp. $\boldsymbol{\varphi}_{j}^{\theta}$, to $\boldsymbol{\varphi}$. The symplectic form of the space of $\boldsymbol{\varphi}$ induces a duality between $U_{j}$ and $U_{j}^{\theta}$. The intersection of $G L\left(l_{j}, \mathbb{C}\right) \times G L\left(l_{j}, \mathbb{C}\right)$ with $S p(N, \mathbb{C})$ is the subgroup of elements compatible with this duality. Its group is isomorphic with $G L\left(l_{i}, \mathbb{C}\right)$. We obtain that the centralizer in $S p(N, \mathbb{C})$ of the image of $\varphi$ is

$$
\left(\prod_{i \in I^{\text {orth }}} S p\left(l_{i}, \mathbb{C}\right)\right) \times\left(\prod_{i \in I^{\text {symp }}} O\left(l_{i}, \mathbb{C}\right)\right) \times\left(\prod_{j \in J} G L\left(l_{j}, \mathbb{C}\right)\right) .
$$

Its group of connected components is therefore isomorphic to $\{ \pm 1\}^{I^{\text {symp }}}$.

Assume now that $\varphi$ takes its values in $O(N, \mathbb{C})$. A similar computation holds for the centralizer in $O(N, \mathbb{C})$ of the image of $\varphi$. We obtain

$$
\left(\prod_{i \in I^{\text {orth }}} O\left(l_{i}, \mathbb{C}\right)\right) \times\left(\prod_{i \in I^{\text {symp }}} S p\left(l_{i}, \mathbb{C}\right)\right) \times\left(\prod_{j \in J} G L\left(l_{j}, \mathbb{C}\right)\right)
$$

But we are more interested by the centralizer in $S O(N, \mathbb{C})$ and not in the full orthogonal group. A little computation shows that it is the subgroup of elements $x$ such that, if $\left(x_{i}\right)_{i \in I^{\text {orth }}}$ are the components of $x$ in the first product, we have $\prod_{i \in I^{\text {orth }}} \operatorname{det}\left(x_{i}\right)^{N_{i}}=1$. The group of connected components of this centralizer is therefore isomorphic to the subgroup of the elements

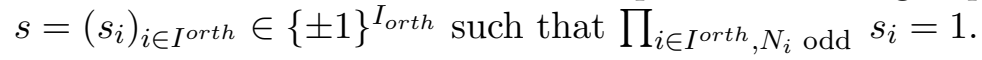

5.2. Definition of some sign. Let $N$ and $N^{\prime}$ be two natural integers, with $N^{\prime}$ even. Let $\varphi: W_{D F} \rightarrow G L(N, \mathbb{C})$ and $\varphi^{\prime}: W_{D F} \rightarrow G L\left(N^{\prime}, \mathbb{C}\right)$ be two homomorphisms as in the preceding section. Assume the image of $\varphi$ is contained in $O(N, \mathbb{C})$ and the image of $\varphi^{\prime}$ is contained in $S p\left(N^{\prime}, \mathbb{C}\right)$. By the Langlands correspondence, we associate to these homomorphisms the representations $\boldsymbol{\pi}(\boldsymbol{\varphi})$ of $G L(N, F)$ and $\boldsymbol{\pi}\left(\boldsymbol{\varphi}^{\prime}\right)$ of $G L\left(N^{\prime}, F\right)$. As in 1.3, we denote by $\delta_{\varphi}$ the quadratic character of $W_{D F}$ defined by $\delta_{\varphi}=\operatorname{det} \circ \varphi$. By Kummer's theory, we can see $\delta_{\varphi}$ as a quadratic character of $F^{\times}$. Define

$$
E\left(\boldsymbol{\varphi}, \varphi^{\prime}\right)=\delta_{\boldsymbol{\varphi}}(-1)^{N^{\prime} / 2} \epsilon\left(\boldsymbol{\pi}(\boldsymbol{\varphi}) \times \boldsymbol{\pi}\left(\boldsymbol{\varphi}^{\prime}\right), 1 / 2, \psi_{F}\right) .
$$

Lemma. The number $E\left(\varphi, \varphi^{\prime}\right)$ belongs to $\{ \pm 1\}$ and does not depend of $\psi_{F}$. 
5.3. Definition of some character. Let $d$ and $d^{\prime}$ be two natural numbers, with $d$ even and $d^{\prime}$ odd. Let $\delta \in F^{\times} / F^{\times, 2}, \varphi \in \Phi^{\text {orth }}(d, \delta)$ and $\varphi^{\prime} \in \Phi^{\text {orth }}\left(d^{\prime}\right)$. As in the preceding sections, we denote by $\varphi$ the composition of $\varphi$ with the embedding $O(d, \mathbb{C}) \rightarrow G L(d, \mathbb{C})$ and by $\varphi^{\prime}$ the composition of $\varphi^{\prime}$ with the embedding $S p\left(d^{\prime}-1, \mathbb{C}\right) \rightarrow G L\left(d^{\prime}-1, \mathbb{C}\right)$. We apply the constructions of 5.1 , denoting by $I^{\text {orth }}, I^{\text {symp }}$ etc. . the data relative to $\varphi$ and by $I^{\prime o r t h}, I^{\prime \text { symp }}$ etc... those relative to $\varphi^{\prime}$. Then we obtain

$$
S\left(\varphi^{\prime}\right) / S\left(\varphi^{\prime}\right)^{0}=\{ \pm 1\}^{I^{\prime s y m p}}
$$

and

$$
S(\varphi) / S(\varphi)^{0} \subset\{ \pm 1\}^{I^{\text {orth }}} .
$$

Denote by $<., .>$ the duality between $\{ \pm 1\}$ and itself, that is

$$
<\eta, \eta^{\prime}>=\left\{\begin{array}{cc}
-1, & \text { if } \eta=\eta^{\prime}=-1 \\
1, & \text { otherwise }
\end{array}\right.
$$

Define a character $\underline{\epsilon}^{\prime}$ of $S\left(\varphi^{\prime}\right) / S\left(\varphi^{\prime}\right)^{0}=\{ \pm 1\}^{I^{\text {symp }}}$ by

$$
\underline{\epsilon}^{\prime}\left(s^{\prime}\right)=\prod_{i^{\prime} \in I^{\prime} \text { symp }}<s_{i^{\prime}}^{\prime}, E\left(\boldsymbol{\varphi}, \varphi_{i^{\prime}}^{\prime}\right)>
$$

for every $s^{\prime}=\left(s_{i^{\prime}}^{\prime}\right)_{i^{\prime} \in I^{\prime}}$ symp $\in\{ \pm 1\}^{I^{\prime s y m p}}$. Define a character $\underline{\epsilon}$ of $\{ \pm 1\}^{I^{\text {orth }}}$ by

$$
\underline{\epsilon}(s)=\prod_{i \in I^{\text {orth }}}<s_{i}, E\left(\boldsymbol{\varphi}_{i}, \varphi^{\prime}\right)>
$$

for every $s=\left(s_{i}\right)_{i \in I^{\text {orth }}} \in\{ \pm 1\}^{I^{\text {orth }}}$. By restriction to $S(\varphi) / S(\varphi)^{0}$, we obtain a character of this group, also denoted by $\underline{\epsilon}$.

We have defined in 4.6 and 4.7 two elements $z_{\varphi} \in S(\varphi) / S(\varphi)^{0}$ and $z_{\varphi^{\prime}} \in S\left(\varphi^{\prime}\right) / S\left(\varphi^{\prime}\right)^{0}$. A little computation shows that

$$
\underline{\epsilon}\left(z_{\varphi}\right)=\underline{\epsilon}^{\prime}\left(z_{\varphi^{\prime}}\right)=E\left(\varphi, \varphi^{\prime}\right) .
$$

5.4. Statement of the local Gross-Prasad conjecture. Let's come back to the situation of 2.1, but changing the notation as explained in 4.1. So the data $d=\operatorname{dim}(V), G, \operatorname{dim}(W), H$ of 2.1 become $d, G, d^{\prime}, G^{\prime}$, if the bigger space is even-dimensional, and become $d^{\prime}, G^{\prime}, d, G$ if the bigger space is odd-dimensional. For smooth irreducible representations $\pi$ of $G(F)$ and $\pi^{\prime}$ of $G^{\prime}(F)$, we denote by $m\left(\pi, \pi^{\prime}\right)$ the multiplicity defined in 2.1 if the first case and the multiplicity denoted in 2.1 by $m\left(\pi^{\prime}, \pi\right)$ in 2.1 in the other case.

Some element $\nu \in F^{\times} / F^{\times, 2}$ occurs in 2.1 when we write explicitely the quadratic form of the space $Z$. In 4.4 , we use some element $\nu$ to define $\mu(G)$. And this sign is used to parametrize the tempered representations of $G(F)$. We assume now that the two elements $\nu$ of 2.1 and 4.4 are the same. The space $V \oplus Z(\nu)$ of 4.4 is the same as $V^{\prime}$, up to addition of hyperbolic 
planes. Then $G^{\prime}(\nu)$ is split if and only if $G^{\prime}$ is split. The definitions imply the equality

$$
\mu(G)=\mu\left(G^{\prime}\right) .
$$

Let $\varphi \in \Phi_{\text {temp }}^{\text {orth }}(d, \delta)$ and let $\varphi^{\prime} \in \Phi_{\text {temp }}^{\text {orth }}\left(d^{\prime}\right)$, where as before $\delta$ is the discriminant of the quadratic form on the even-dimensional space. To those parameters are associated packets $\Pi^{G}(\varphi)$ and $\Pi^{G^{\prime}}\left(\varphi^{\prime}\right)$ of tempered representations of $G(F)$ and $G^{\prime}(F)$. Those packets are themselves parametrized by the sets of characters $\mathcal{E}^{\mu(G)}(\varphi)$ and $\mathcal{E}^{\mu\left(G^{\prime}\right)}\left(\varphi^{\prime}\right)$.

REMARK. We assume the parametrizations fixed such that the equalities of propositions 4.6 and 4.7 are satisfied. There is no logical problem here: these propositions and the theorem below follow from the same proof, but the propositions are proved firstly.

Applying the contructions of 5.3, we define two characters $\underline{\epsilon}$ and $\underline{\epsilon}^{\prime}$. The equality 5.3(1) and the equality above show that there are two possibilities:

(1) if $E\left(\boldsymbol{\varphi}, \varphi^{\prime}\right)=\mu(G)=\mu\left(G^{\prime}\right)$, then $\underline{\epsilon}$ belongs to $\mathcal{E}^{\mu(G)}(\varphi)$ and $\underline{\epsilon}^{\prime}$ belongs to $\mathcal{E}^{\mu\left(G^{\prime}\right)}\left(\varphi^{\prime}\right)$

(2) if $E\left(\varphi, \varphi^{\prime}\right)=-\mu(G)=-\mu\left(G^{\prime}\right)$, then $\underline{\epsilon}$ does not belong to $\mathcal{E}^{\mu(G)}(\varphi)$ and $\underline{\epsilon}^{\prime}$ does not belong to $\mathcal{E}^{\mu\left(G^{\prime}\right)}\left(\varphi^{\prime}\right)$.

TheOREM. (i) If $E\left(\boldsymbol{\varphi}, \varphi^{\prime}\right)=\mu(G)=\mu\left(G^{\prime}\right)$, then $m\left(\pi(\varphi, \underline{\epsilon}), \pi\left(\varphi^{\prime}, \underline{\epsilon}^{\prime}\right)\right)=$ 1 and $m\left(\pi, \pi^{\prime}\right)=0$ for every $\left(\pi, \pi^{\prime}\right) \in \Pi^{G}(\varphi) \times \Pi^{G^{\prime}}\left(\varphi^{\prime}\right)$ such that $\left(\pi, \pi^{\prime}\right) \neq$ $\left(\pi(\varphi, \underline{\epsilon}), \pi\left(\varphi^{\prime}, \underline{\epsilon}^{\prime}\right)\right)$.

(ii) If $E\left(\varphi, \varphi^{\prime}\right)=-\mu(G)=-\mu\left(G^{\prime}\right)$, then $m\left(\pi, \pi^{\prime}\right)=0$ for every $\left(\pi, \pi^{\prime}\right) \in \Pi^{G}(\varphi) \times \Pi^{G^{\prime}}\left(\varphi^{\prime}\right)$.

Cf. [W4] théorème 4.9. Let's recall the theorem is conditional: we assume true the conjectures of Section 4, and in addition some results coming from the twisted local trace formula.

The theorem proves the local Gross-Prasad conjecture. Of course, it implies the theorem $\mathrm{A}$ and $\mathrm{B}$ of 1.5 and 1.6. There is two pairs $\left(G_{i}, H_{i}\right)$ and $\left(G_{a}, H_{a}\right)$ of groups in these theorems, and not one as in the statement above. We explain that in the remark 2 of the following section.

5.5. An idea of the proof. To state the preceding theorem, we started from given groups $G$ and $G^{\prime}$. In order to prove it, it is better to start from more abstract data. Those data are:

- an element $\nu \in F^{\times} / F^{\times, 2}$ fixed once and for all;

- two natural integers $d$ and $d^{\prime}$, with $d$ even and $d^{\prime}$ odd;

- an element $\delta \in F^{\times} / F^{\times, 2}$, with $\delta=1$ if $d=0$;

- a sign $\mu \in\{ \pm 1\}$. 
Let $\varphi \in \Phi_{\text {temp }}^{\text {orth }}(d, \delta), \varphi^{\prime} \in \Phi_{\text {temp }}^{\text {orth }}\left(d^{\prime}\right), \epsilon \in \mathcal{E}^{\mu}(\varphi)$ and $\epsilon^{\prime} \in \mathcal{E}^{\mu}\left(\varphi^{\prime}\right)$. We can define the multiplicity $m\left(\varphi, \epsilon ; \varphi^{\prime}, \epsilon^{\prime}\right)$ as follows. Using the elementary classification's theory of quadratic forms over local fields, we see that, up to isometry, there exists exactly two spaces provided with non-degenerate quadratic forms satisfying the conditions of 2.1, where $\nu$ is our choosen $\nu$, such that the even-dimensional space has dimension $d$ and discriminant $\delta$, the odd-dimensional space has dimension $d^{\prime}$ and the special orthogonal group $G^{\prime}$ of the odd-dimensional space is split if $\mu=1$ and non-split if $\mu=-1$. We apply the constructions of the preceding sections to those spaces. The pairs $(\varphi, \epsilon)$ and $\left(\varphi^{\prime}, \epsilon^{\prime}\right)$ defines representations $\pi(\varphi, \epsilon)$ of $G(F)$ and $\pi\left(\varphi^{\prime}, \epsilon^{\prime}\right)$ of $G^{\prime}(F)$ and we let

$$
m\left(\varphi, \epsilon ; \varphi^{\prime}, \epsilon^{\prime}\right)=m\left(\pi(\varphi, \epsilon), \pi\left(\varphi^{\prime}, \epsilon^{\prime}\right)\right) .
$$

Remarks. (1) For defining the representation $\pi(\varphi, \epsilon)$ of $G(F)$, we need to consider $S O(d, \mathbb{C})$ as the Langlands dual group of $G$ and for that reason, we need to fix some additional data, cf. 4.7. To change those data can replace $\pi(\varphi, \epsilon)$ by a representation conjugate by an element of the full orthogonal group. But fortunately, we see easily that the multiplicity above does not change. So the additional data are not necessary for our problem.

(2) To obtain the situation of theorems A and B, we fix only $\nu, d, d^{\prime}$ and $\delta$. Choosing $\mu=1$, the preceding construction gives two groups that we denote by $G_{i}$ and $G_{i}^{\prime}$ (or $G_{i}$ and $H_{i}$ with the notation of Section 1). Choosing $\mu=-1$, the construction gives other groups $G_{a}$ and $G_{a}^{\prime}$. The group $G_{i}$ is quasi-split and the group $G_{i}^{\prime}$ is split. The group $G_{a}$ is an inner form of $G_{i}$ (that can be also quasi-split and in this case isomorphic to $G_{i}$ ) and the group $G_{a}^{\prime}$ is a non-quasi-split inner form of $G_{i}^{\prime}$.

The problem is to compute $m\left(\varphi, \epsilon ; \varphi^{\prime}, \epsilon^{\prime}\right)$ in terms of $\epsilon$-factors. Let $\varphi$ and $\varphi^{\prime}$ be as before and let $s \in S(\varphi) / S(\varphi)^{0}$ and $s^{\prime} \in S\left(\varphi^{\prime}\right) / S\left(\varphi^{\prime}\right)^{0}$. Define

$$
m^{\mu}\left(\varphi, s ; \varphi^{\prime}, s^{\prime}\right)=\sum_{\epsilon \in \mathcal{E}^{\mu}(\varphi), \epsilon^{\prime} \in \mathcal{E}^{\mu}\left(\varphi^{\prime}\right)} \epsilon(s) \epsilon^{\prime}\left(s^{\prime}\right) m\left(\varphi, \epsilon ; \varphi^{\prime}, \epsilon^{\prime}\right)
$$

By elementary Fourier's inversion, to compute all terms $m\left(\varphi, \epsilon ; \varphi^{\prime}, \epsilon^{\prime}\right)$ is equivalent to compute all terms $m^{\mu}\left(\varphi, s ; \varphi^{\prime}, s^{\prime}\right)$. Let $d_{1}, d_{2}, d_{1}^{\prime}, d_{2}^{\prime}$ be four integers such that $d_{1}$ and $d_{2}$ are even and $d_{1}+d_{2}=d, d_{1}^{\prime}$ and $d_{2}^{\prime}$ are odd and $d_{1}^{\prime}+d_{2}^{\prime}=d^{\prime}+1$, and let $\delta_{1}, \delta_{2}$ be two elements of $F^{\times} / F^{\times, 2}$ such that $\delta_{1} \delta_{2}=\delta$ and, if $d_{i}=0$ for $i=1$ or 2 , then $\delta_{i}=1$. For $i=1$ or 2 , let $\varphi_{i} \in \Phi_{\text {temp }}^{\text {orth }}\left(d_{i}, \delta_{i}\right)$ and $\varphi_{i}^{\prime} \in \Phi_{\text {temp }}^{\text {orth }}\left(d_{i}^{\prime}\right)$. To $\left(\varphi_{1}, \varphi_{2}\right)$, we associate as in 4.7 an element $\varphi \in \Phi_{\text {temp }}^{\text {orth }}(d, \delta)$ and an element $s \in S(\varphi) / S(\varphi)^{0}$ (as above, we need to choose some additional data, but this choice is inessential). To $\left(\varphi_{1}^{\prime}, \varphi_{2}^{\prime}\right)$, we associate as in 4.6 an element $\varphi^{\prime} \in \Phi_{\text {temp }}^{\text {orth }}\left(d^{\prime}\right)$ and an element $s^{\prime} \in$ $S\left(\varphi^{\prime}\right) / S\left(\varphi^{\prime}\right)^{0}$. The application that, to the data $d_{1}, d_{2}, \ldots, \varphi_{2}^{\prime}$, associates the data $\varphi, s, \varphi^{\prime}, s^{\prime}$ is surjective (not injective, but this doesn't matter). Then, in order to compute $m^{\mu}\left(\varphi, s ; \varphi^{\prime}, s^{\prime}\right)$, we can assume that $\varphi, s, \ldots$ are deduced 
from fixed data $d_{1}, d_{2}, \ldots, \varphi_{2}^{\prime}$. For $i, j \in\{1,2\}$, Define

$$
S\left(\varphi_{i}, \varphi_{j}^{\prime}\right)=2 m^{+}\left(\varphi_{i}, 1_{\varphi_{i}} ; \varphi_{j}^{\prime}, 1_{\varphi_{j}^{\prime}}\right)-1
$$

Recall we have introduced some scalars $\gamma^{\mu}\left(\varphi_{1}, \varphi_{2}\right)$ and $\gamma^{\mu}\left(\varphi_{1}^{\prime}, \varphi_{2}^{\prime}\right)$ in 4.6 and 4.7 .

Proposition. We have the equality

$$
\begin{aligned}
& \gamma^{\mu}\left(\varphi_{1}, \varphi_{2}\right) \gamma^{\mu}\left(\varphi_{1}^{\prime}, \varphi_{2}^{\prime}\right) m^{\mu}\left(\varphi, s ; \varphi^{\prime}, s^{\prime}\right) \\
& \quad=\frac{1}{2}\left(S\left(\varphi_{1}, \varphi_{1}^{\prime}\right) S\left(\varphi_{2}, \varphi_{2}^{\prime}\right)+\mu S\left(\varphi_{1}, \varphi_{2}^{\prime}\right) S\left(\varphi_{2}, \varphi_{1}^{\prime}\right)\right) .
\end{aligned}
$$

Sketch OF THE PROOF. We associate to $\varphi$ and $\varphi^{\prime}$ some groups $G$ and $G^{\prime}$ as above. We associate to $d_{1}, d_{2}, \delta_{1}$ and $\delta_{2}$ the groups $G_{1}$ and $G_{2}$ of 4.7 and to $d_{1}^{\prime}$ and $d_{2}^{\prime}$ the groups $G_{1}^{\prime}$ and $G_{2}^{\prime}$ of 4.6 . The term $m^{\mu}\left(\varphi, s ; \varphi^{\prime}, s^{\prime}\right)$ is a weighted sum of multiplicities that can be computed by the integral formula of 2.5. Then the whole term $m^{\mu}\left(\varphi, s ; \varphi^{\prime}, s^{\prime}\right)$ is a sum over tori of integrals of functions, that are weighted sums of the functions appearing in 2.5. In fact, those weighted sums are

$$
\sum_{\epsilon \in \mathcal{E}^{\mu}(\varphi)} \epsilon(s) c_{\pi(\varphi, \epsilon)}
$$

and

$$
\sum_{\epsilon^{\prime} \in \mathcal{E}^{\mu}\left(\varphi^{\prime}\right)} \epsilon^{\prime}\left(s^{\prime}\right) c_{\pi\left(\varphi^{\prime}, \epsilon^{\prime}\right)}
$$

They can be deduced from the characters $\theta^{G}(\varphi, s)$ and $\theta^{G^{\prime}}\left(\varphi^{\prime}, s^{\prime}\right)$ introduced in 4.6 and 4.7. Using the conjectures of these sections, they can be computed in terms of the characters $\theta^{G_{1}}\left(\varphi_{1}, 1_{\varphi_{1}}\right) \times \theta^{G_{2}}\left(\varphi_{2}, 1_{\varphi_{2}}\right)$ and $\theta^{G_{1}^{\prime}}\left(\varphi_{1}^{\prime}, 1_{\varphi_{1}^{\prime}}\right) \times$ $\theta^{G_{2}^{\prime}}\left(\varphi_{2}^{\prime}, 1_{\varphi_{2}^{\prime}}\right)$ (here we need to slip the scalars $\gamma^{\mu}\left(\varphi_{1}, \varphi_{2}\right)$ and $\left.\gamma^{\mu}\left(\varphi_{1}^{\prime}, \varphi_{2}^{\prime}\right)\right)$. We obtain an integral formula computing the left member of the proposition, using those characters. The same thing holds for the right member, applying the same methods to the four pairs of groups $\left(G_{1}, G_{1}^{\prime}\right),\left(G_{2}, G_{2}^{\prime}\right),\left(G_{1}, G_{2}^{\prime}\right)$ and $\left(G_{2}, G_{1}^{\prime}\right)$. The proposition results from comparison of the two integral formulas obtained in this way

REMARK. Stictly speaking, it is not always possible to attach to $G_{1}$, $G_{2}, G_{1}^{\prime}$ and $G_{2}^{\prime}$ spaces $V_{1}, V_{2}, V_{1}^{\prime}, V_{2}^{\prime}$, provided with quadratic forms, such that the assumptions of 2.1 are satisfied for the four pairs $\left(V_{i}, V_{j}^{\prime}\right), i, j=$ 1,2 . That is, we cannot use the same $\nu$ for the four pairs. But a term $S\left(\varphi_{i}, \varphi_{j}^{\prime}\right)$ depends only on the $L$-packets $\Pi^{G_{i}}\left(\varphi_{i}\right)$ and $\Pi^{G_{j}^{\prime}}\left(\varphi_{j}^{\prime}\right)$ and not on the parametrizations of those packets. That is, it does not depend on $\nu$.

We must to compute the terms $S\left(\varphi_{i}, \varphi_{j}^{\prime}\right)$. For that, we can forget the indices $i$ and $j$ and to compute $S\left(\varphi, \varphi^{\prime}\right)$ defined as above by

$$
S\left(\varphi, \varphi^{\prime}\right)=2 m^{+}\left(\varphi, 1_{\varphi} ; \varphi^{\prime}, 1_{\varphi^{\prime}}\right)-1 .
$$


So $\mu=1$ and the underlying groups are quasi-split. We introduce as in 4.6 and 4.7 the representations $\boldsymbol{\pi}\left(\boldsymbol{\varphi}_{>}^{\prime}\right)$ of $G L\left(d^{\prime}, F\right)$ and $\boldsymbol{\pi}(\boldsymbol{\varphi})$ of $G L(d, F)$, and the scalars $c_{>}\left(\varphi^{\prime}\right)$ and $c(\varphi)$. Recall the scalar $\zeta(\varphi)$ of the first assertion of proposition 4.7 (that is proved directly).

Proposition. We have the equality

$$
S\left(\varphi, \varphi^{\prime}\right)=\zeta(\varphi) c(\varphi) c_{>}\left(\varphi^{\prime}\right) \epsilon\left(\boldsymbol{\pi}(\varphi) \times \boldsymbol{\pi}\left(\varphi_{>}^{\prime}\right), 1 / 2, \psi_{F}\right) .
$$

SketCH OF THE PROOF. We associate to $\varphi$ and $\varphi^{\prime}$ the same groups $G$ and $G^{\prime}$ as above. The left member $S\left(\varphi, \varphi^{\prime}\right)$ can again be computed by an integral formula where appear functions deduced from the characters $\theta^{G}\left(\varphi, 1_{\varphi}\right)$ and $\theta^{G^{\prime}}\left(\varphi^{\prime}, 1_{\varphi^{\prime}}\right)$. As in 4.5 , we associate to $G$ a group $\mathbf{G}$ and a twisted space $\tilde{\mathbf{G}}$, then to $\varphi$ the function $\theta_{\tilde{\boldsymbol{\pi}}(\boldsymbol{\varphi})}$ on $\tilde{\mathbf{G}}(F)$. As in 4 .4, we associate to $G^{\prime}$ a group $\mathbf{G}^{\prime}$ and a twisted space $\tilde{\mathbf{G}}^{\prime}$, then to $\varphi^{\prime}$ the function $\theta_{\tilde{\boldsymbol{\pi}}\left(\varphi_{>}^{\prime}\right)}$ on $\tilde{\mathbf{G}}^{\prime}(F)$. Using the conjectures of 4.6 and 4.7 , we can replace the characters $\theta^{G}\left(\varphi, 1_{\varphi}\right)$ and $\theta^{G^{\prime}}\left(\varphi^{\prime}, 1_{\varphi^{\prime}}\right)$ by $c(\varphi) \theta_{\tilde{\boldsymbol{\pi}}(\boldsymbol{\varphi})}$ and $c_{>}\left(\varphi^{\prime}\right) \theta_{\tilde{\boldsymbol{\pi}}\left(\boldsymbol{\varphi}_{>}^{\prime}\right)}$. Then $S\left(\varphi, \varphi^{\prime}\right)$ is computed by an integral formula where appear functions deduced from these last characters. Using the proposition 3.4, we see that the same is true for the right member of the proposition. The equality of the two members results from comparison of the two integral formulas obtained in this way.

REMARK. In a first step, this proof gives a more complicated formula because an auxiliary parameter $\boldsymbol{\nu}$ appears in proposition 3.4. But we can choose arbitrarily this parameter. Because the final formula cannot depend on this choice, this implies some relations between the various characters appearing in the formula and these relations can be used to simplify the formula as in the above statement.

Using the two preceding propositions, we obtain an equality between $m^{\mu}\left(\varphi, s ; \varphi^{\prime}, s^{\prime}\right)$ and an expression that contains some $\epsilon$-factors and various scalars as $\gamma^{\mu}\left(\varphi_{1}, \varphi_{2}\right), c\left(\varphi_{1}\right)$, etc... To determine the scalars, we consider particular cases where some terms can be computed. For instance, assume $d=0, d_{2}^{\prime}=1$ and $\mu=+1$. Then all terms depending on $\varphi, \varphi_{1}, \varphi_{2}$ and $\varphi_{2}^{\prime}$ disappears. The only scalars that appear are $c_{>}\left(\varphi^{\prime}\right)$ and $\gamma^{+}\left(\varphi^{\prime},-\right)$ (the term $\varphi_{2}^{\prime}$ disappears and $\left.\varphi^{\prime}=\varphi_{1}^{\prime}\right)$. The transfer from $G_{1}^{\prime}=G^{\prime}$ to $G^{\prime}$ is the identity and this implies that $\gamma^{+}\left(\varphi^{\prime},-\right)=1$. The $\epsilon$-factors are associate to pairs of representations of linear groups, where at least one of the groups is $G L(0)$. Then these $\epsilon$-factors are equal to 1 . Our equality reduces to

$$
m^{+}\left(-,-; \varphi^{\prime}, 1_{\varphi^{\prime}}\right)=c_{>}\left(\varphi^{\prime}\right)
$$

But, by definition, the left member is a true sum of multiplicities, then is a natural integer. And the right member is a complex number of absolute value 1. Then the two members are equal to 1 , proving $c_{>}\left(\varphi^{\prime}\right)=1$. More complicated particular cases can be used to determine the others scalars. After 
computation of these scalars, our formula becomes and equality expressing $m^{\mu}\left(\varphi, s ; \varphi^{\prime}, s^{\prime}\right)$ in terms of $\epsilon$-factors and some computations prove that it is equivalent to the formula of the theorem 5.4.

REMARK. As above, we can apply the constructions to the cases $d=0$ or $d^{\prime}=1$. We obtain the following results. Assume $\mu\left(G^{\prime}\right)=1$ and denote by $\epsilon_{1}^{\prime}$ the identity element of $\left(S\left(\varphi^{\prime}\right) / S\left(\varphi^{\prime}\right)^{0}\right)^{\vee}$. Then the representation $\pi\left(\varphi^{\prime}, \epsilon_{1}^{\prime}\right)$ is the unique element of $\Pi^{G^{\prime}}\left(\varphi^{\prime}\right)$ that is generic (cf. 6.2 for the definition). Assume $\mu(G)=1$ and denote by $\epsilon_{1}$ the identity element of $\left(S(\varphi) / S(\varphi)^{0}\right)^{\vee}$. Then the representation $\pi\left(\varphi, \epsilon_{1}\right)$ is the unique element of $\Pi^{G}(\varphi)$ that is generic, for a type of Whittaker model depending on $\nu$.

\section{The case of generic packets}

6.1. Definition of non-tempered packets. Let $V, q, G$ be as in 4.4. Let $\varphi \in \Phi^{\text {orth }}(d, \delta)$. We don't assume $\varphi$ tempered. There exists a Levi subgroup $\hat{M}$ of $S O(d, \mathbb{C})$, in the sense of 2.7 , an homomorphism $\varphi_{\text {temp }}: W_{D F} \rightarrow \hat{M}$ that is tempered $\left(\varphi_{\text {temp }}\left(W_{F}\right)\right.$ is relatively compact), and an homomorphism $\hat{\chi}$ from $W_{D F}$ to the center $Z(\hat{M})$, such that:

- the homomorphism $\hat{\chi}$ is positive; that means that for every rational character $x^{*}: Z(\hat{M}) \rightarrow \mathbb{C}^{\times}, x^{*} \circ \hat{\chi}$ takes real positive values; this implies $\hat{\chi}$ is in fact defined on the quotient $W_{F} / I_{F}$ of $W_{D F}$;

- the homomorphism $\varphi$ is equivalent to $\hat{\chi} \otimes \varphi_{\text {temp }}$;

- if another triple $\hat{M}_{1}, \varphi_{\text {temp }, 1}$ and $\hat{\chi}_{1}$ satisfy the same properties, then $\hat{M}_{1} \subset \hat{M}$.

In general, the group $\hat{M}$ defines by duality a Levi subgroup $M$ of $G$. It is not true in some particular case. We ignore temporarily this difficulty. The group $M$ is a product of groups $G L(n)$ and (possibly) one special orthogonal group of the even-dimensional case. We can apply to this last group the conjecture of 4.7 and to the components $G L(n)$ the known correspondence of Langlands. Then the homomorphism $\varphi_{\text {temp }}$ defines a packet $\Pi^{M}\left(\varphi_{\text {temp }}\right)$ of smooth irreducible tempered representations of $M(F)$. The character $\hat{\chi}$ defines a character $\chi^{M}$ of $M(F)$. We can choose a parabolic subgroup $P$ of $G$, of Levi component $M$, such that $\hat{\chi}$ satisfies relatively to $P$ the positivity condition required for applying the Langlands quotient theorem. Then this theorem says that, for all $\tau \in \Pi^{M}\left(\varphi_{\text {temp }}\right)$, the induced representation $\operatorname{Ind}_{P}^{G}\left(\chi^{M} \otimes \tau\right)$ has a unique irreducible quotient. Denote $\mathcal{L}\left(\chi^{M} \otimes \tau\right)$ this quotient. By definition, the $L$-packet $\Pi^{G}(\varphi)$ is the set of such representations $\mathcal{L}\left(\chi^{M} \otimes \tau\right)$, when $\tau$ describes $\Pi^{M}\left(\varphi_{\text {temp }}\right)$.

Let's come back to the particular case where $M$ does not exist. This case happens when $G$ is not quasi-split and $\hat{M}$ is a product of groups $G L(n, \mathbb{C})$ only, without a special orthogonal group. In this case, we define $\Pi^{G}(\varphi)=\emptyset$.

We see easily that $S(\varphi)$ is equal to the centralizer of the image of $\varphi_{\text {temp }}$ in $\hat{M}$. Then $\Pi^{G}(\varphi)$ is still parametrized by $\mathcal{E}^{\mu(G)}(\varphi)$. 
Now let $V^{\prime}, q^{\prime}, G^{\prime}$ be as in 4.3 and let $\varphi^{\prime} \in \Phi^{\text {orth }}\left(d^{\prime}\right)$. We define the packet $\Pi^{G^{\prime}}\left(\varphi^{\prime}\right)$ by a similar construction.

6.2. Definition of generic packets. We consider two sets of data $V_{i}^{\prime}$, $q_{i}^{\prime}, G_{i}^{\prime}$ and $V_{a}^{\prime}, q_{a}^{\prime}, G_{a}^{\prime}$ as in 4.3 , of the same odd dimension $d^{\prime}$ and the same discriminant, and we assume $\mu\left(G_{i}^{\prime}\right)=1, \mu\left(G_{a}^{\prime}\right)=-1$ (in other words, $G_{i}^{\prime}$ is split and $G_{a}^{\prime}$ is not-split). Let $\varphi^{\prime} \in \Phi^{\text {orth }}\left(d^{\prime}\right)$. The $L$-packet $\Pi^{G_{i}^{\prime}}\left(\varphi^{\prime}\right)$ is parametrized by $\mathcal{E}^{+}\left(\varphi^{\prime}\right)$ and the $L$-packet $\Pi^{G_{a}^{\prime}}\left(\varphi^{\prime}\right)$ is parametrized by $\mathcal{E}^{-}\left(\varphi^{\prime}\right)$. As in 1.3 , let $\Pi\left(\varphi^{\prime}\right)=\Pi^{G_{i}^{\prime}}\left(\varphi^{\prime}\right) \sqcup \Pi^{G_{a}^{\prime}}\left(\varphi^{\prime}\right)$.

Because $G_{i}^{\prime}$ is split, we can fix a Borel subgroup $B^{\prime}$ of $G_{i}^{\prime}$, defined over $F$. Denote by $U^{\prime}$ the unipotent radical of $B^{\prime}$. Fix a character $\psi$ of $U^{\prime}(F)$. We assume that, for all parabolic subgroup $P^{\prime}$ of $G_{i}^{\prime}$ defined over $F$ such that $B^{\prime} \subset P^{\prime} \neq G_{i}^{\prime}, \psi$ is not trivial on $U^{P^{\prime}}(F)$, where $U^{P^{\prime}}$ is the unipotent radical of $P^{\prime}$. Define the space $\mathcal{W}_{\psi}$ of locally constant functions $f: G_{i}^{\prime}(F) \rightarrow \mathbb{C}$ such that $f(u g)=\psi(u) f(g)$ for all $u \in U^{\prime}(F)$ and $g \in G_{i}^{\prime}(F)$ and such that $|f|$ is of compact support on $U^{\prime}(F) \backslash G_{i}^{\prime}(F)$. The group $G_{i}^{\prime}(F)$ acts by right translations on this space. For a smooth irreducible representation $\pi^{\prime}$ of $G_{i}^{\prime}(F)$, we say $\pi^{\prime}$ is generic if it is equivalent to a representation in a subspace of $\mathcal{W}_{\psi}$. This notion does not depend on $\psi$ because all $\psi$ 's are conjugate by the action of $B^{\prime}(F)$. We say that $\Pi\left(\varphi^{\prime}\right)$ is generic if $\Pi^{G_{i}^{\prime}}\left(\varphi^{\prime}\right)$ contains a generic representation.

Now, we consider two sets of data $V_{i}, q_{i}, G_{i}$ and $V_{a}, q_{a}, G_{a}$ as in 4.4, of the same even dimension $d$ and the same discriminant $\delta$, and we assume $\mu\left(G_{i}\right)=1, \mu\left(G_{a}\right)=-1$ (the parameter $\nu$ used in the definition of these signs being always fixed). The group $G_{i}^{\prime}$ is quasi-split. Let $\varphi \in \Phi^{\text {orth }}(d, \delta)$ and let $\Pi(\varphi)=\Pi^{G_{i}}(\varphi) \sqcup \Pi^{G_{a}}(\varphi)$. Suppressing the ', we define as before the notion of genericity of a smooth irreducible representation of $G_{i}(F)$ or of the packet $\Pi(\varphi)$. Here, there is a problem because the possible characters $\psi$ are not all conjugate by the action of $B(F)$. A representation of $G_{i}(F)$ can be generic for one choice of $\psi$ and not generic for another choice. But there is no problem for the packets: we see that, if the packet $\Pi^{G_{i}}(\varphi)$ contains a representation generic for one choice, then for any choice, it contains a generic representation.

6.3. The theorem for generic $L$-packets. We consider the same data as in 5.4, but we remove the assumption that $\varphi$ and $\varphi^{\prime}$ are tempered. So we have only $\varphi \in \Phi^{\text {orth }}(d, \delta)$ and $\varphi^{\prime} \in \Phi^{\text {orth }}\left(d^{\prime}\right)$. Using the definitions of 6.1, the statement of theorem 5.5 has still a meaning. Without additional assumptions, this theorem is false.

TheOREM (joint with Moeglin). The assertions of theorem 5.5 become true if $\Pi(\varphi)$ and $\Pi\left(\varphi^{\prime}\right)$ are generic.

That is the theorem $\mathrm{C}$ of 1.6. Note that a tempered $L$-packet is generic: as explained in the last remark of 5.5, that follows from theorem 5.5 applied 
to the cases $d=0$ or $d^{\prime}=1$. So the preceding theorem is stronger as theorem 5.5.

I give a sketch of the proof. To $\varphi$, we associate as in 6.1 an homomorphism $\varphi_{\text {temp }}$ and, for an index $\sharp=i$ or $a$, a Levi subgroup $M_{\sharp}$, a parabolic subgroup $P_{\sharp}$ of $G_{\sharp}$ and a character $\chi^{M_{\sharp}}$ of $M_{\sharp}(F)$ (assuming that $M_{\sharp}$ exists). Similarly, to $\varphi^{\prime}$, we associate $\varphi_{\text {temp }}^{\prime}, M_{\sharp}^{\prime}, P_{\sharp}^{\prime}$ and $\chi^{M_{\sharp}^{\prime}}$.

We have a first result. Let $\tau \in \Pi^{M_{\sharp}}\left(\varphi_{\text {temp }}\right)$ and $\tau^{\prime} \in \Pi^{M_{\sharp}^{\prime}}\left(\varphi_{\text {temp }}^{\prime}\right)$. Assume the representations $\operatorname{Ind}_{P_{\sharp}}^{G_{\sharp}}\left(\chi^{M_{\sharp}} \otimes \tau\right)$ and $\operatorname{Ind}_{P_{\sharp}^{\prime}}^{G_{\sharp}^{\prime}}\left(\chi^{M_{\sharp}^{\prime}} \otimes \tau^{\prime}\right)$ are irreducible. Then

$$
m\left(\operatorname{Ind} d_{P_{\sharp}}^{G_{\sharp}}\left(\chi^{M_{\sharp}} \otimes \tau\right), \operatorname{Ind} d_{P_{\sharp}^{\prime}}^{G_{\sharp}^{\prime}}\left(\chi^{M_{\sharp}^{\prime}} \otimes \tau^{\prime}\right)\right)=m\left(\tau, \tau^{\prime}\right),
$$

the last multiplicity being defined as in 2.7. This comes easily, using the proposition 2.8 .

The second ingredient is more subtle. We fix a character $\psi$ as in 6.2. Because $\varphi_{\text {temp }}$ is tempered, there is a unique representation in $\Pi^{M_{i}}\left(\varphi_{\text {temp }}\right)$ that is generic. Denote it by $\tau_{W h}$.

THEOREM. The three conditions are equivalent:

(i) $\Pi(\varphi)$ is generic;

(ii) the representation $\operatorname{Ind}_{P_{i}}^{G_{i}}\left(\chi^{M_{i}} \otimes \tau_{W h}\right)$ is irreducible;

(iii) for $\sharp=i$ or $a$ and for all $\tau \in \Pi^{M_{\sharp}}\left(\varphi_{\text {temp }}\right)$, the representation $\operatorname{Ind} d_{P_{\sharp}}^{G_{\sharp}}\left(\chi^{M_{\sharp}} \otimes \tau\right)$ is irreducible.

Cf. [MW] corollary 2.14. The equivalence between (i) and (ii) was a conjecture of Shahidi, and was proved by Heiermann and Muic ([HM]). The new point is the equivalence with (iii). The rather winding proof uses the fine study by Moeglin of the relations between Jacquet modules and endoscopy.

A similar result holds for the groups $G_{\sharp}^{\prime}$.

Now, assume $\Pi(\varphi)$ and $\Pi\left(\varphi^{\prime}\right)$ are generic. By the two results above, we have the equality

$$
m\left(\mathcal{L}\left(\chi^{M_{\sharp}} \otimes \tau\right), \mathcal{L}\left(\chi^{M_{\sharp}^{\prime}} \otimes \tau^{\prime}\right)\right)=m\left(\tau, \tau^{\prime}\right)
$$

for $\sharp=i$ or $a$ and for all $\tau \in \Pi^{M_{\sharp}}\left(\varphi_{t e m p}\right)$ and $\tau^{\prime} \in \Pi^{M_{\sharp}^{\prime}}\left(\varphi_{t e m p}^{\prime}\right)$. Then the theorem above comes from the theorem 5.5 of the tempered case.

\section{References}

[AGRS] A. Aizenbud, D. Gourevitch, S. Rallis, G. Schiffmann: Multiplicity one theorems, Annals of Math. 172 (2010), p. 1407-1434

[A] J. Arthur: Endoscopic classification of representations: orthogonal and symplectic groups, in preparation

[GGP1] W.T. Gan, B. Gross, D. Prasad: Restrictions of representations of classical groups: examples, preprint 2009

[GGP2] — : Symplectic local root numbers, central critical L-values, and restriction problems in the representation theory of classical groups, preprint 2009 
[GP] B. Gross, D. Prasad: On the decomposition of a representation of $\mathrm{SO}_{n}$ when restricted to $S_{n-1}$, Can. J. of Math. 44 (1992), p.974-1002

[GR] B. Gross, M. Reeder: From Laplace to Langlands via representations of orthogonal groups, Bull. AMS 43 (2006), p.163-205

[HC] Harish-Chandra: Admissible invariant distributions on reductive p-adic groups, notes by S. DeBacker and P. Sally, University lecture series 16, AMS 1999

[HT] M. Harris, R. Taylor: On the geometry and cohomology of some simple Shimura varieties, Ann. of Math. Studies 151, PUP 2002

[HM] V. Heiermann, G. Muic: The standard modules conjecture, Math. Zeit. 255 (2007), p.19-37

$[\mathrm{H}] \quad$ G. Henniart: Une preuve simple des conjectures de Langlands pour $G L(n)$ sur un corps p-adique, Invent. Math. 139 (2000), p.439-456

[II] A. Ichino, T. Ikeda: On the periods of automorphic forms on special orthogonal groups and the Gross-Prasad conjecture, preprint

[JPSS] H. Jacquet, I. Piatetski-Shapiro, J. Shalika: Rankin-Selberg convolutions, American J. of Math. 105 (1983), p.367-464

[MW] C. Moeglin, J.-L. Waldspurger: La conjecture locale de Gross-Prasad pour les groupes spéciaux orthogonaux: le cas général, preprint 2009

[W1] J.-L. Waldspurger: Une formule intégrale reliée à la conjecture locale de GrossPrasad, Compositio Math. 146 (2010), p.1180-1290

[W2] - Une formule intégrale reliée à la conjecture locale de Gross-Prasad, $2^{\text {ème }}$ partie: extension aux représentations tempérées, to appear in Astérisque

[W3] - Calcul d'une valeur d'un facteur $\epsilon$ par une formule intégrale, preprint 2009

[W4] - La conjecture locale de Gross-Prasad pour les représentations tempérées des groupes spéciaux orthogonaux, preprint 2009 
